JOURNAL OF THE

AMERICAN MATHEMATICAL SOCIETY

Volume 23, Number 2, April 2010, Pages 491-533

S 0894-0347(09)00653-5

Article electronically published on December 2, 2009

\title{
A LINDEMANN-WEIERSTRASS THEOREM FOR SEMI-ABELIAN VARIETIES OVER FUNCTION FIELDS
}

\author{
DANIEL BERTRAND AND ANAND PILLAY
}

\section{Contents}

1. Introduction 492

1.1. Statement of the results 492

1.2. Organisation of the proofs 495

2. Differential algebraic preliminaries 497

2.1. The twisted tangent bundle 498

2.2. Algebraic $D$-groups and logarithmic derivatives 499

2.3. $D$-modules and the connection $\partial_{L G}$ on $L G \quad 500$

2.4. Algebraic $D$-groups and differential algebraic groups 501

3. Almost semi-abelian $D$-groups 501

3.1. Almost semi-abelian varieties and $D$-groups 501

3.2. Isoconstant $D$-groups 505

4. Further ingredients and special cases 506

4.1. The socle theorem 506

4.2. On the semi-constant part and descent of $(\mathbf{H X})_{K}$

4.3. Special cases: Ax and the theorem of the kernel 508

5. Proofs of the main results 509

5.1. Proof of Theorem $1.3 \quad 509$

5.2. Dropping $(\mathbf{H G})_{0}$

5.3. A counterexample 511

5.4. Extension to several variables 512

6. $K$-largeness and a differential Galois-theoretic proof 514

6.1. $K$-large algebraic $D$-groups $\quad 514$

6.2. Differential Galois theory 516

Appendix: Exponentials on algebraic D-groups 517

A. Setting

B. The functors $T_{\partial}, L$ on algebraic groups

C. Algebraic $D$-groups and $\partial \ell_{n_{\mathbf{G}}} \quad 518$

D. The connection $\partial_{L G}$ on $L G \quad 519$

E. The exponential map on $\mathbf{G}^{\text {an }}$

F. The connection $\exp _{\mathbf{G}}^{*}\left(\partial \ell n_{\mathbf{G}}\right)$ on $L \mathbf{G}^{a n}$

Received by the editors October 24, 2008.

2000 Mathematics Subject Classification. Primary 12H05, 14K05, 03C60, 34M15, 11J95.

Key words and phrases. Algebraic independence, differential algebraic groups, logarithmic derivatives, Gauss-Manin connections, differential Galois theory.

The second author was supported by a Marie Curie Chair and an EPSRC grant.

(C)2009 American Mathematical Society Reverts to public domain 28 years from publication 
G. $\exp _{\mathbf{G}}^{*}\left(\partial \ell n_{\mathbf{G}}\right)=\partial_{L \mathbf{G}}$

H. Gauss-Manin: $\nabla_{L \mathbf{G}, \partial}=\exp _{\mathbf{G}}^{*}\left(\partial \ell n_{\mathbf{G}}\right)$

I. Almost semi-abelian $D$-groups: An analytic approach 526 section*.99

J. Manin's theorem 527

K. Chai's sharpening $\quad 529$

Acknowledgements 532

References

\section{INTRODUCTION}

1.1. Statement of the results. Let $G$ be a commutative algebraic group defined over the algebraic closure $\mathbb{Q}^{\text {alg }}$ of $\mathbb{Q}$ in $\mathbb{C}$, let $L G$ be its Lie algebra, and let $\exp _{G}$ : $L G(\mathbb{C}) \rightarrow G(\mathbb{C})$ be the exponential map on the Lie group $G^{a n}$ deduced from $G$ after extension of scalars to $\mathbb{C}$. Further, let $x$ be a point in $L G\left(\mathbb{Q}^{\text {alg }}\right)$ and assume that

$(\mathbf{H X})_{\mathbb{Q}^{a l g}}$ : for any proper algebraic subgroup $H / \mathbb{Q}^{\text {alg }}$ of $G, x \notin L H\left(\mathbb{Q}^{\text {alg }}\right)$.

The classical Lindemann-Weierstrass theorem then states that if $G$ is an algebraic torus, the field of definition of the point $y=\exp _{G}(x) \in G(\mathbb{C})$ satisfies

$$
\operatorname{tr.deg} .\left(\mathbb{Q}^{\text {alg }}(y) / \mathbb{Q}^{\text {alg }}\right)=\operatorname{dim}(G) .
$$

One may wonder under which conditions on $x$ such a result extends to more general groups $G$. However, the answer is known (and with the same hypothesis) only when $G$ is isogenous to a power of an elliptic curve with complex multiplication (Philippon, Wüstholz; cf. [16], Theorem 6.25).

In the present paper we study the analogous problem where $\mathbb{Q}^{\text {alg }}$ is replaced by the algebraic closure $K^{a l g}$ of the function field $K=\mathbb{C}(S)$ of a smooth algebraic curve $S / \mathbb{C}$. Here is a typical corollary of our main theorem.

Let $\pi: \mathbf{B} \rightarrow S$ be a semi-abelian scheme of constant toric rank, with generic fiber $B / K$ and Lie algebra $L B / K$, let $A / K$ be the maximal abelian variety occuring as a quotient of $B$, and let $\left(A_{0}, \tau\right)$ be the $K / \mathbb{C}$ trace of $A / K$, where we assume for simplicity that $A_{0}$ is included in $A$. By base change to a finite cover of $S$, we may assume that it is also the $K^{\text {alg }} / \mathbb{C}$-trace of $A / K^{\text {alg }}$, and we will just call it the $\mathbb{C}$ trace, or the constant part, of $A$. Further, let $B_{0}$ be the pull-back of $A_{0}$ to $B$. This is a semi-abelian variety, which is defined over $K$ and does not in general descend to $\mathbb{C}$; we call $B_{0}$ the semi-constant part of $B$. Finally, let $\tilde{\mathbf{B}}$ be the universal vectorial extension of $\mathbf{B}$, with generic fiber $\tilde{B} / K$. Consider the exponential morphisms of analytic sheaves attached to the group schemes $\mathbf{B}^{a n}, \tilde{\mathbf{B}}^{a n}$ over $S^{a n}$ :

$$
\exp _{\mathbf{B}}: L \mathbf{B}^{a n} \longrightarrow \mathbf{B}^{a n}, \exp _{\tilde{\mathbf{B}}}: L \tilde{\mathbf{B}}^{a n} \longrightarrow \tilde{\mathbf{B}}^{a n} .
$$

Further, let $x$ be a point in $L B(K)$. Restricting $S$ if necessary, we may assume that $x$ extends to a section $\mathbf{x} \in L \mathbf{B}(S)$ and lift it to a section $\tilde{\mathbf{x}}$ of $L \tilde{\mathbf{B}}(S)$, for which we set $\mathbf{y}=\exp _{\mathbf{B}}(\mathbf{x}) \in \mathbf{B}^{a n}\left(S^{a n}\right), \tilde{\mathbf{y}}=\exp _{\tilde{\mathbf{B}}}(\tilde{\mathbf{x}}) \in \tilde{\mathbf{B}}^{a n}\left(S^{a n}\right)$. Abbreviating these analytic sections as $y=\exp _{B}(x), \tilde{y}=\exp _{\tilde{B}}(\tilde{x})$, and making use of the same notations over all covers of $S$, we will prove: 
Corollary 1.1. Let $B / K^{\text {alg }}$ be a semi-abelian variety, and let $x$ be a point in $L B\left(K^{\text {alg }}\right)$. Assume that

$\left(\mathbf{H B}_{0}\right)$ : the semi-constant part $B_{0}$ of $B$ is defined over $\mathbb{C}$;

$(\mathbf{H X} / \mathbf{B})_{K^{a l g}}$ : for any proper semi-abelian subvariety $H$ of $B, x \notin L H\left(K^{a l g}\right)+$ $L B_{0}(\mathbb{C})$.

Further, let $\tilde{x} \in L \tilde{B}\left(K^{\text {alg }}\right)$ be a lift of $x$. Then, the fields of definition of the points $y=\exp _{B}(x), \tilde{y}=\exp _{\tilde{B}}(\tilde{x})$ satisfy

$$
\operatorname{tr} . \operatorname{deg} \cdot\left(K^{a l g}(\tilde{y}) / K^{a l g}\right)=\operatorname{dim}(\tilde{B}),
$$

and in particular tr.deg. $\left(K^{\text {alg }}(y) / K^{\text {alg }}\right)=\operatorname{dim}(B)$.

(We recall that by rigidity, all abelian subvarieties $H$ of $B$ are defined over $K^{\text {alg }}$.)

Consider for instance the case where $B=A$ is an abelian variety, so that $\left(\mathbf{H B}_{0}\right)$ is automatically satisfied. When $A$ is a power of an elliptic curve, Corollary 1.1 says that if $\wp$ is an elliptic function with a non-constant invariant $j \in$ $\mathbb{C}(z)$ and zeta function $\zeta$, and if $x_{1}(z), \ldots, x_{n}(z)$ are $\mathbb{Z}$-linearly independent algebraic functions, then the $2 n$ analytic functions defined on some open domain of $\mathbb{C}$ by $\wp\left(x_{1}(z)\right), \ldots, \wp\left(x_{n}(z)\right), \zeta\left(x_{1}(z)\right), \ldots, \zeta\left(x_{n}(z)\right)$ are algebraically independent over $\mathbb{C}(z)$. In the opposite case where $j \in \mathbb{C}$, this holds only if $x_{1}, \ldots, x_{n}$ are linearly independent, modulo $\mathbb{C}$, over the ring of multipliers of $\wp$. When, as in the latter case, the full abelian variety is constant $\left(A=A_{0}\right)$, such results follow from Ax's work on the Schanuel conjecture; cf [2], Theorem 3, [19], 44, and in the elliptic case, [7].

Remark 1.2. (i) A similar result holds for the exponential morphism of the formal group $\hat{\tilde{B}}$ of $\tilde{B}$ at the origin; cf. Appendix, Remark G.6. Namely, assuming that the section $\tilde{\mathbf{x}} \in L \tilde{\mathbf{B}}(S)$ vanishes at a given point $s_{0}$ of $S$, and denoting by $\hat{S}_{s_{0}}$ the formal completion of $s_{0}$ in $S$, we can deduce from our methods a direct proof that $\hat{\tilde{\mathbf{y}}}=\exp _{\hat{\mathbf{\mathbf { B }}}}(\hat{\tilde{\mathbf{x}}}) \in \hat{\tilde{\mathbf{B}}}\left(\hat{S}_{s_{0}}\right)$ has transcendence degree $\operatorname{dim}(\tilde{B})$ over $K$. Note that this formal setting is the framework used by $\mathrm{Ax}$ in the constant case; cf. [2, Theorem 3. In an analogous way, one can replace in this corollary $\mathbb{C}$ by the $p$-adic field $\mathbb{C}_{p}$, with a suitable convergence condition on the point $x$.

(ii) (Several variables.) Let $S^{\prime} / \mathbb{C}$ be an algebraic variety of dimension $n \geq 1$, and let $K^{\prime}=\mathbb{C}\left(S^{\prime}\right)$ be its field of rational functions. As is often the case in complex algebraic geometry, Corollary 1.1. over the one-dimensional function field $K / \mathbb{C}$ implies the same statement for any semi-abelian variety $B^{\prime}$ over $K^{\prime}$ whose semi-constant part $B_{0}^{\prime}$ is constant. See Theorem 5.3 of Section 5.4 for a precise formulation of this generalization and for the reduction of its proof to Corollary 1.1. See also Remark 1.5 (ii) below for a different approach to this generalization.

As in Ax's initial work on the toric case, Corollary 1.1 will follow from consideration of the differential relations or equations satisfied by $(x, y)$ such that $\exp (x)=y$. But contrary to Ax's setting, the ambient semi-abelian variety $B$ will not be constant here, and we will in general only be able to find the appropriate differential equations on the universal vectorial extension $\tilde{B}$ of $B$ (and its Lie algebra). Our main theorem, on solutions $\tilde{y}$ of $\partial \ell n_{\tilde{B}}(\tilde{y})=\partial_{L \tilde{B}}(\tilde{x})$, is Theorem 1.4 below. The inductive nature of the proof (passing to quotients) will force us into the more general category of almost semi-abelian D-groups, and our main technical result is Theorem 1.3 below. More precisely, and referring to the following sections of the paper for the underlying notions, we will study the following problem. 
Now let $K=K^{\text {alg }}$ be the algebraic closure of a function field in one variable over $\mathbb{C}$, let $\partial$ be a non-trivial derivation on $K$, let $\mathcal{U}$ be some universal differential extension of $K$, and let $K^{\text {diff }}$ be a differential closure of $K$ in $\mathcal{U}$. More will be said about $K^{\text {diff }}$ and $\mathcal{U}$ at the beginning of Section 2. For now we just mention that $K^{\text {diff }}$ and $K$ have the same field of constants, namely $\mathbb{C}$.

Let $G / K$ be a connected commutative algebraic D-group (cf. 9], 31 and Section 2 below); i.e., $G$ is a connected commutative algebraic group over $K$, equipped with an extension to $\mathcal{O}_{G}$ of the derivation $\partial$, which respects the group structure of $G$. Denoting Lie algebras by $L$, we write

$$
\partial \ell n_{G}: G \rightarrow L G
$$

for the corresponding logarithmic derivative on $G$ (cf. Section 2). This is a "first order differential algebraic" homomorphism which takes $G(\mathcal{U})$ onto $L G(\mathcal{U})$, and likewise takes $G\left(K^{\text {diff }}\right)$ onto $L G\left(K^{\text {diff }}\right)$, but will be far from surjective at the level of $K$-points. The kernel of $\partial \ell n_{G}$, denoted $G^{\partial}$ when the $D$-group structure on $G$ is assumed, is a "differential algebraic group" defined over $K$, and for any differential extension field $K^{\prime}$ of $K$ (including $K^{\text {diff }}$ and $\mathcal{U}$ ), we can speak of the group $G^{\partial}\left(K^{\prime}\right)$ of its $K^{\prime}$-points. We will write

$$
\partial_{L G}: L G \rightarrow L G
$$

for the canonical connection, contracted with $\partial$, which $\partial \ell_{G}$ induces on $L G$ and which we can again view as a differential algebraic endomorphism of $L G$, surjective at the level of $\mathcal{U}$-points. This is discussed in detail in the Appendix of this paper. For instance, when $G$ is the universal vectorial extension of an abelian variety $A$, $\partial_{L G}$ coincides with the dual of the standard Gauss-Manin connection on $H_{d R}^{1}(A / K)$. Again we write $(L G)^{\partial}$ for the kernel of $\partial_{L G}$, namely the space of vectors horizontal for the connection $\partial_{L G}$.

We consider the differential relation

$$
\partial \ell n_{G}(y)=\partial_{L G}(x)
$$

where $(x, y) \in(L G \times G)(\mathcal{U})$, and proceed to compute the transcendence degree of $K(y)$ over $K$ under the assumption that $x$ is $K$-rational. As will later become apparent, this is the natural algebraic description of the "Lindemann-Weierstrass case" of the Schanuel conjecture: with some abuse of notation, the rough idea is that whenever $\exp _{G}(x)$ is well-defined, we have

$$
\partial \ell n_{G}\left(\exp _{G}(x)\right)=\partial_{L G}(x)
$$

(see the proof of Corollary 1.1 at the end of this Introduction and Section $\mathrm{G}$ of the Appendix), so that up to addition by elements $x_{0} \in(L G)^{\partial}(\mathcal{U}), y_{0} \in G^{\partial}(\mathcal{U})$,

$$
\partial \ell n_{G}(y)=\partial_{L G}(x) \Leftrightarrow y-y_{0}=\exp _{G}\left(x-x_{0}\right) .
$$

In the present paper, we do not discuss the case where $y$ is $K$-rational and $x$ is the unknown, nor the general case where both $x$ and $y$ are unknown. These would respectively correspond to the functional analogues of the Grothendieck and Schanuel-André conjectures; cf. [4.

We make the necessary assumption that our algebraic $D$-group $G$ admits no nonzero vectorial quotient, and we thereby restrict ourselves to almost semi-abelian $D$-groups, as defined in Section 3. These are the quotients

$$
G=\tilde{B} / U
$$


by a vectorial $D$-subgroup $U$ of the universal extension $\tilde{B}$ of a semi-abelian variety $B$ (the latter being endowed with its unique $D$-group structure). Furthermore, we will assume that $B$ satisfies Hypothesis $(\mathbf{H B})_{0}$ of Corollary 1.1 and will rename this hypothesis as

$(\mathbf{H G})_{0}$ : the semi-constant part $B_{0}$ of the maximal semi-abelian quotient $B$ of $G$ is actually constant.

Let $B_{(0)}$ be the constant part of $B$, i.e., the maximal semiabelian subvariety of $B$ isomorphic to one defined over $\mathbb{C}$. In general, $B_{(0)}$ is contained in $B_{0}$, and hypothesis $(\mathbf{H G})_{0}$ means that they coincide. In particular, it is automatically satisfied if the maximal abelian quotient $A$ of $B$ is traceless (in which case $B_{(0)}=B_{0}$ is the toric part of $B$ ) or if $B$ is the product of the abelian variety $A$ by a torus. Our main technical result can then be stated as follows.

Theorem 1.3. Let $G$ be an almost semi-abelian D-group, defined over $K$, which satisfies Hypothesis $(\mathbf{H G})_{0}$, and let $x$ be a point in $L G(K)$. Assume that

$(\mathbf{H X})_{K}: x \notin L H(K)+(L G)^{\partial}(K)$ for any proper algebraic subgroup $H / K$ of $G$.

Let $y \in G(\mathcal{U})$ be a solution of the differential equation $\partial \ln _{G}(y)=\partial_{L G}(x)$. Then

$$
\text { tr.deg. }(K(y) / K)=\operatorname{dim}(G) \text {. }
$$

We will give (see Section 5.3) an example showing that the $(\mathbf{H G})_{0}$ hypothesis in Theorem 1.3 cannot be dropped. On the other hand, Theorem 5.2 shows that hypothesis $(\mathbf{H G})_{0}$ can be dropped, but at the expense of strengthening the hypothesis $(\mathbf{H X})_{K}$ to $(\mathbf{H X}): x \notin L H(\mathcal{U})+L G^{\partial}(\mathcal{U})$ for any proper algebraic subgroup $H / K$ of $G$. However we tend to prefer the $(\mathbf{H X})_{K}$ hypothesis because, as witnessed by Corollary 1.1, the results it yields are closer in spirit to the number theoretic case.

The proofs of Theorem 1.3 and these other results will be given in Section 5 of the paper. In [4] it was suggested that differential Galois theory in the most general form may be useful in this function field Lindemann-Weierstrass context. Under an additional assumption of " $K$-largeness" on the algebraic $D$-group $G$ (which actually implies $\left.(\mathbf{H G})_{0}\right)$, such a Galois-theoretic proof of Theorem 1.3 is in fact possible, and is given in Section 6 .

When $G=\tilde{B}$ is the universal extension of a semi-abelian variety $B$ as in Corollary 1.1, Theorem 1.3 yields our main result:

Theorem 1.4. Let $B / K$ be a semi-abelian variety, and let $x$ be a point in $L B(K)$. Assume that

$\left(\mathbf{H B}_{0}\right)$ : the semi-constant part $B_{0}$ of $B$ is defined over $\mathbb{C}$;

$(\mathbf{H X} / \mathbf{B})_{K}$ : for any proper semi-abelian subvariety $H$ of $B, x \notin L H(K)+$ $L B_{0}(\mathbb{C})$.

Further, let $\tilde{x} \in L \tilde{B}(K)$ be a lift of $x$, and let $\tilde{y} \in \tilde{B}(\mathcal{U})$ be a solution of the differential equation $\partial \ell n_{\tilde{B}}(\tilde{y})=\partial_{L \tilde{B}}(\tilde{x})$. Then,

$$
\operatorname{tr} . \operatorname{deg} \cdot(K(\tilde{y}) / K)=\operatorname{dim}(\tilde{B}) .
$$

Since the the almost semi-abelian $D$-groups of Theorem 1.3 are quotients of such $D$-groups $\tilde{B}$, it is conversely clear that Theorem 1.4 implies Theorem 1.3 (details are given below).

1.2. Organisation of the proofs. The proof of Theorem 1.3 (and variants) in Section 5 has a number of ingredients and draws on several sources. On the one hand there is differential algebraic geometry (and model theory) which provides 
the notions of algebraic $D$-group, logarithmic derivative, etc., in which our results are phrased in the main body of the paper, as well as the powerful "socle theorem" which is closely related to the function field Mordell-Lang conjecture in characteristic 0 and in the present context facilitates an inductive proof. Then there are results originating with $\mathrm{Ax}$ dealing with the case of $G$ defined over $\mathbb{C}$. Finally, from algebraic geometry we make use of the Manin-Coleman-Chai theorem of the kernel, as well as the Griffiths-Schmid-Deligne theorem of the fixed part and Deligne's semi-simplicity theorem. To be able to draw on these various sources we need to know at least the compatibility of the different languages and constructions. Among the issues is the relation between the logarithmic derivative on the universal extension $\tilde{A}$ of an abelian variety $A$ defined over $K$ and the Gauss-Manin connection on $H_{d R}^{1}(A / K)=$ dual of $L(\tilde{A})$. So our rather extensive appendix, Exponentials on Algebraic D-groups, is devoted to clarifying some of these issues, although they are probably well-known. A discussion of the "theorem of the kernel" also appears there.

In Sections 2 and 3 we introduce and discuss algebraic $D$-groups, differential algebraic groups, $D$-modules, and almost semiabelian $D$-groups, as well as logarithmic derivatives, in the context of Kolchin's differential algebraic geometry. Section 3 contains a few new observations. Section 4 presents the main tools (including the "socle theorem") and special cases which will be used in the proof of the main theorem (Theorem 1.3 above). As already stated, the proof of the main theorem, plus a variant as well as a (counter)example, are given in Section 5, while Section 6 provides another proof in the special case where the algebraic $D$-group $G$ is $K$ large, based on differential Galois theory in place of the socle theorem. As for the Appendix, its main results have been gathered in a Conclusion before Section J.

We conclude this Introduction by discussing the mutual relations between Theorem 1.3, Theorem 1.4 and Corollary 1.1, in particular showing how to deduce Corollary 1.1 from Theorem 1.3.

Theorem $1.3 \Leftrightarrow$ Theorem 1.4

Since in the projection $\tilde{B} \rightarrow \tilde{B} / U=G$ transcendence degrees decrease at most by the dimension $U$ of the kernel, Theorem 1.3 on a general $G$ is equivalent to its special case for $\tilde{B}$. Since Hypothesis $\left(\mathbf{H B}_{0}\right)$ is just a renaming of $\left(\mathbf{H G}_{0}\right)$, it remains, given a point $x \in L B(K)$ and a lift $\tilde{x}$ of $x$ to $L \tilde{B}(K)$, to check that $x$ satisfies hypothesis $(\mathbf{H X} / \mathbf{B})_{K}$ of Theorem 1.4 with respect to $L B$ if and only if $\tilde{x}$ satisfies the corresponding hypothesis, say $(\mathbf{H} \tilde{\mathbf{X}})_{K}$, of Theorem 1.3 with respect to $L \tilde{B}$. Now, we will show in Lemma 4.2(i) (see Corollary H.5 (ii) of the Appendix in the case $B$ is an abelian variety) that under $\left(\mathbf{H B}_{0}\right)$, the universal vectorial extension $\tilde{B}_{0} \subset \tilde{B}$ of $B_{0}$, which is then clearly defined over $\mathbb{C}$, satisfies

$$
(L \tilde{B})^{\partial}(K)=L \tilde{B}_{0}(\mathbb{C}) .
$$

Furthermore, $\tilde{B}$ is an essential extension of $B$. That is to say, any proper algebraic subgroup of $\tilde{B}$ projects onto a proper algebraic subgroup of $B$.

So, suppose that there exists a proper algebraic subgroup $H^{\prime} / K$ of $\tilde{B}$ with projection $H$ on $B$, such that $\tilde{x}$ lies in $L H^{\prime}(K)+L \tilde{B}_{0}(\mathbb{C})$. Projecting to $B$, we deduce from $(\mathbf{H X} / \mathbf{B})_{K}$ that $H$ fills up $B$, and $H^{\prime}$ cannot be proper. Conversely, suppose that $x$ lies in $L H(K)+L B_{0}(\mathbb{C})$ for some proper semi-abelian subvariety $H$ of $B$, with inverse image $H^{\prime}$ in $\tilde{B}$. Then $H^{\prime}$ is a proper algebraic subgroup of $\tilde{B}$ over $K$ satisfying $\tilde{x} \in L H^{\prime}(K)+(L \tilde{B})^{\partial}(K)$, which contradicts $(\mathbf{H} \tilde{\mathbf{X}})_{K}$. 
Theorem $1.4 \Rightarrow$ Corollary 1.1

We go back to the notation before the statement of this corollary, but now denote by $K=\mathbb{C}(S)^{\text {alg }}$ the algebraic closure of the field $\mathbb{C}(S)$.

So, let $\tilde{\mathbf{y}}:=\exp _{\tilde{\mathbf{B}}}(\tilde{\mathbf{x}}) \in \tilde{\mathbf{B}}\left(S^{a n}\right)$ be the image of $\tilde{\mathbf{x}}$ under the exponential morphism of the group scheme $\tilde{\mathbf{B}}^{a n} / S^{a n}$. By one of the compatibilities proven in the Appendix (cf. Proposition G.1), its logarithmic derivative $\partial \ell_{\tilde{B}}$, extended to $\tilde{\mathbf{B}} / S$, satisfies

$$
\partial \ell n_{\tilde{\mathbf{B}}} \tilde{\mathbf{y}}=\partial \ell n_{\tilde{\mathbf{B}}}\left(\exp _{\tilde{\mathbf{B}}} \tilde{\mathbf{x}}\right)=\partial_{L \tilde{\mathbf{B}}} \tilde{\mathbf{x}}
$$

Viewing the field of meromorphic functions on $S^{a n}$ as a subfield of $\mathcal{U}$, we have thus constructed a solution $\tilde{y} \in \tilde{B}(\mathcal{U})$ of the differential equation $\partial \ln _{\tilde{B}} \tilde{y}=\partial_{L \tilde{B}} \tilde{x}$ with $\tilde{x} \in \tilde{B}(K)$. Theorem 1.4 then implies that the transcendence degree of $K(\tilde{\mathbf{y}})=K(\tilde{y})$ over $K$ is equal to $\operatorname{dim}(\tilde{B})$. As for the last statement of the corollary, it again follows from the fact that in the projection from $\tilde{B}$ to $B$, transcendence degrees can at most decrease by the dimension of the kernel.

Notice that contrary to $\tilde{B}$, the semi-abelian variety $B$ admits in general no $D$ group structure, so that the relation $y=\exp _{B}(x)$ cannot be expressed directly on $B$ in differential algebraic terms. In other words, even for the study of the point $y$, lifting to $\tilde{B}$ is forced onto us in order to allow for the techniques of proofs of Theorem 1.3.

Remark 1.5. (i) We are aware that statements such as Corollary 1.1 can often be proved by purely analytic means, by using the order of growth of $\exp _{\tilde{\mathbf{B}}}(\tilde{\mathbf{x}})$ at the essential singularities given by the poles of the rational section $\tilde{x} \in L \tilde{B}(K)$. But as mentioned in Remark 1.2, the scope of our methods is broader. Corollary 1.1 is merely an illustration, while the true object of study of this article is the differential relation $(*)$ in differential fields.

(ii) (Several variables.) In parallel with Remark 1.2 (ii) and the material in Section 5.4, let us mention that another approach to generalizing our results to function fields of higher-dimensional varieties is to directly adapt the differential algebraic Theorem 1.3 and so also Theorem 1.4 to the several variables context. So now $K^{\prime}=\mathbb{C}\left(S^{\prime}\right)$ is a function field in $n \geq 1$ variables, which we equip with a set $\Delta=\left\{\partial_{1}, \ldots, \partial_{n}\right\}$ of commuting derivations such that $\mathbb{C}$ is the field of $\Delta$-constants of $K^{\prime}$. The notion of an algebraic $D$-group over $K^{\prime}$, which already appears in [9], will now be with respect to $\Delta$ rather than a single derivation, and the commuting of the $\partial_{i}$ will be reflected in suitable integrability requirements on the " $D$-structure" and on the system of PDE's generalizing $(*)$. We intend to come back to this in a later work, but expect the proof of the corresponding Theorem 1.3 to go through with essentially no new ingredient.

\section{Differential algebraic preliminaries}

The context here is the differential algebra or differential algebraic geometry of Ritt and Kolchin, as developed in Kolchin's books [20, 21]. We refer to Buium's books [9] (especially Chapter 5) and [10, to the second author's paper [31] and to Malgrange's monograph 24] for the specific notions needed for the current paper, although we will give a brief account in this section.

We fix a universal differential field $(\mathcal{U}, \partial)$ of characteristic 0 in which all differential fields we discuss are assumed to embed. We denote by $\mathcal{C}$ the field of constants of $\mathcal{U}$, by $K$ an algebraically closed differential field (differential subfield of $\mathcal{U}$ ), by 
$C_{K}$ its field of constants and by $K^{\text {diff }}$ a differential closure of $K$. Of course in our main applications $K$ will have transcendence degree 1 over $C_{K}=\mathbb{C}$. Here is a quick description of $\mathcal{U}$ and differential closures: a differential field $L$ is said to be differentially closed if any finite system of differential polynomial equations over $L$ in unknowns $x_{1}, \ldots, x_{n}$ which has a common solution in some differential field extending $L$ already has a solution in $L$. Differentially closed fields exist. More precisely, fix an uncountable cardinal $\kappa$. Then $\mathcal{U}$ will be a differentially closed field of cardinality $\kappa$ with the following property: whenever $L_{1}<L_{2}$ are differential fields of cardinality $<\kappa$ and $f: L_{1} \rightarrow \mathcal{U}$ is an embedding (of differential fields), then $f$ extends to $g: L_{2} \rightarrow \mathcal{U}$. In our context, $\kappa$ is assumed to be strictly greater than the cardinality of our base differential field $K$. A differential closure of $K$ is a differentially closed field extending $K$ with the property that it embeds over $K$ into any differentially closed field containing $K$. Again a differential closure of $K$ exists and is unique up to isomorphism over $K$. So $K^{\text {diff }}$ denotes a differential closure of $K$ inside $\mathcal{U}$.

The geometric objects of Kolchin's theory are "differential algebraic varieties", which are given locally as common solution sets in $\mathcal{U}^{n}$ of finite systems of differential polynomial equations. They form a category whose morphisms are easily defined and will be termed "differential". Furthermore, we say that a differential algebraic variety $X$ is defined over $K$ if its defining equations have coefficients in $K$. One can then view $X$ as a functor from the category of differential $K$-algebras to sets. Likewise one has the notion of a differential algebraic group. However, the differential algebraic groups we consider will all be given explicitly as differential algebraic subgroups of algebraic groups (which is no loss of generality; see Corollary 4.2 of [29]). If $X$ is a differential algebraic variety (in particular if it is an algebraic variety) defined over a subfield of $\mathcal{U}$, we will often identify $X$ with its set $X(\mathcal{U})$ of $\mathcal{U}$-points.

2.1. The twisted tangent bundle. If $X \subseteq \mathcal{U}^{n}$ is an affine algebraic variety and $a \in X$, we can apply $\partial$ to the coordinates of $x$ to obtain a point $\partial(x) \in \mathcal{U}^{n}$. This depends of course on the chosen embedding $X \subseteq \mathcal{U}^{n}$, but it can be viewed in an intrinsic way as a (differential rational rather than rational) section of a certain twisted tangent bundle $T_{\partial}(X)$ of $X$, which we now describe. We assume $X$ smooth, (geometrically) irreducible, and defined over $K$. If $X \subseteq \mathcal{U}^{n}$ is affine, then $T_{\partial}(X)=\left\{(a, u) \in \mathcal{U}^{2 n}: a \in X\right.$ and $\sum\left(\partial f / \partial x_{i}\right)(a) u_{i}+f^{\partial}=0$ for $f$ ranging over generators of $I(X)\}$. Here $f^{\partial}$ is obtained from $f$ by applying $\partial$ to the coefficients of $f$. For arbitrary $X$, take a covering by open affines $U_{j}$ and piece together the $T_{\partial}\left(U_{i}\right)$ using the transition maps in the obvious way, to obtain $T_{\partial}(X)$. So if $X$ is defined over $C_{K}$, then $T_{\partial}(X)$ coincides with the tangent bundle $T(X)$ of $X$. In general the definition shows $T_{\partial}(X)$ to be a torsor (over $X$ ) under $T(X)$. For $X$ affine one sees from the Leibniz rule that if $a \in X$, then $(a, \partial(a)) \in T_{\partial}(X)$. In the general case this makes sense too, and with abuse of notation we call $\partial: X \rightarrow T_{\partial}(X)$ the corresponding (differential regular) section. If $s: X \rightarrow T_{\partial}(X)$ is a regular section, defined over $K$, then we obtain a (finite-dimensional) differential algebraic variety $(X, s)^{\partial}=\{a \in X: \partial(a)=s(a)\}$, defined over $K$. Finite-dimensionality means the following: Suppose $X \subseteq \mathcal{U}^{n}$ is an affine differential algebraic variety defined over $K$. We call $X$ finite-dimensional if there is a positive integer $m$ such that for any point $a \in X$, the differential field $K\langle a\rangle$ generated by $K$ and $a$ has transcendence degree at most $m$ over $K$. So if $X$ is an algebraic variety (i.e., with no additional differential 
equations) and not a point, then $X$ is infinite-dimensional. On the other hand, clearly our $(X, s)^{\partial}$ above is finite-dimensional, as for $a \in(X, s)^{\partial}, K\langle a\rangle=K(a)$.

In Sections A and B of the Appendix, a geometric account of these and the next notions is given when the base $K$ is replaced by a curve $S$ over $\mathbb{C}$, and $\partial \mid K$ by a vector field on $S$. In fact (like $T$ ) $T_{\partial}$ is a functor: if $\phi: X \rightarrow Y$ is a morphism over $K$ (of smooth irreducible varieties), then $T_{\partial}(\phi): T_{\partial}(X) \rightarrow T_{\partial}(Y)$ is given in local coordinates by $T \phi+\phi^{\partial}$.

2.2. Algebraic $D$-groups and logarithmic derivatives. If $G$ is now a connected algebraic group defined over $K$, then because $T_{\partial}$ is a functor, $T_{\partial}(G)$ (also denoted $T_{\partial} G$ ) can be naturally equipped with the structure of an algebraic group over $K$, and the canonical projection $\pi: T_{\partial}(G) \rightarrow G$ is a homomorphism of algebraic groups whose kernel is canonically isomorphic to the Lie algebra $L G$ of $G$. Indeed, this kernel is $\left(T_{\partial} G\right)_{e}$, the fibre of $T_{\partial} G$ above the identity $e$ of $G$, which we have seen is a principal homogeneous space for $T(G)_{e}=L G$ over $K$. Now the identity element of $T_{\partial} G$, that is the $K$-rational point $(e, \partial(e))$, gives rise to an identification of $T_{\partial} G_{e}$ and $L G$. (See Section 2 of [31.)

By an algebraic $D$-group (or just a $D$-group) over $K$, we mean a pair $(G, s)$ where $G$ is an algebraic group over $K$ and $s: G \rightarrow T_{\partial}(G)$ is a regular section defined over $K$ which is also a homomorphism. Algebraic $D$-groups are objects of algebraic geometry. Giving $G$ the structure $s$ of an algebraic $D$-group over $K$ is equivalent to extending the derivation $\partial \mid K$ to a derivation of the structure sheaf of $G$ over $K$ which respects the group operation, and this is how they were first defined by Buium 9 .

We will restrict our attention to commutative algebraic groups $G$. In this case $T_{\partial}(G)$ is also commutative, and we will write its group law using additive notation. One can check that $\partial: G \rightarrow T_{\partial}(G)$ is also a homomorphism, so if $(G, s)$ is an algebraic $D$-group, then $\partial-s$ (where - is meant in the sense of the group $T_{\partial}(G)$ ) is a differential regular homomorphism from $G$ to $L G$, which we call the logarithmic derivative associated to $(G, s)$ and which is written as $\partial \ell n_{(G, s)}$ or $\partial \ell n_{G}$ when $s$ is understood. Consistent with earlier notation we write $(G, s)^{\partial}$, or just $G^{\partial}$, for the kernel of this logarithmic derivative. $(G, s)^{\partial}$ is a (finite-dimensional) differential algebraic group. If $(G, s)$ is defined over $K$, then for any differential field $F$ containing $K$, we have $\partial \ell n_{(G, s)}: G(F) \rightarrow L G(F)$. For $F=\mathcal{U}$ or $K^{\text {diff }}$, this map is surjective.

Two special cases of an algebraic $D$-group should be familiar. The first is when $G$ is defined over $C_{K}$ and $s: G \rightarrow T(G)$ is the 0 -section. Then the corresponding logarithmic derivative reduces to the classical one of Kolchin, so for example if $G$ is a torus, it is just $\partial(g) \cdot g^{-1}$. Moreover, $G^{\partial}(\mathcal{U})$ is just $G(\mathcal{C})$.

The second is when $G=\mathbb{G}_{a}^{n}$. Then $L G=G$ and $T(G)=G \times G$. A regular section $s$ defined over $K$ is of the form $x \mapsto(x, A x)$, where $A$ is an $n \times n$ matrix over $K$, and we write $x \in G$ as a column vector. Hence the corresponding logarithmic derivative is $x \mapsto \partial(x)-A x$ from $G$ to $G$. This is precisely a $D$-module structure on $\mathcal{U}^{n}$ or on $K^{n}$ if we restrict to $K$-points, in other words a linear differential system. Indeed, a $D$-module defined over $K$ is by definition a $K$-vector space $V$ (that is, a commutative unipotent group over $K$ as we are in characteristic 0) together with an additive homomorphism $D_{V}: V \rightarrow V$ satisfying, with $\partial$, the Leibniz rule. The algebraic group $V$ is isomorphic to some $\mathbb{G}_{a}^{n}$ over $K$, so the $D$-module structure is as above the logarithmic derivative for some algebraic $D$-group structure on $\mathbb{G}_{a}^{n}$. 
2.3. $D$-modules and the connection $\partial_{L G}$ on $L G$. Here we discuss the $D$-module structure induced on the Lie algebra of an algebraic $D$-group $(G, s)$, which yields our map $\partial_{L G}$ referred to in the Introduction. This is a rather delicate aspect of the paper, in terms of compatibilities, and much of the Appendix is devoted to it. Here we give an "algebraic" definition based on Section 5 of 32 and point out some equivalences. First suppose $X$ to be a smooth irreducible affine variety over $K$ with coordinates $x=\left(x_{1}, \ldots, x_{n}\right)$. Recall from Section 2.1 that the tangent bundle of $X$ is defined by equations for $X$ together with $d f \cdot y=0$ for $f$ ranging over generators of the ideal of $X$ (where $d f \cdot y=\sum_{i=1, \ldots, n}\left(\partial f(x) / \partial x_{i}\right) y_{i}=0$ represents the (vertical) differential $d_{G / K} f$ of $f$ ). On the other hand the twisted tangent bundle of $X$ is defined by equations defining $X$ together with $d f(x) \cdot y+f^{\partial}(x)$ for $f$ generating the ideal of $X$. So we choose $(x, u)$ as coordinates of $T(X)$ and $(x, y)$ as coordinates of $T_{\partial}(X)$. Likewise let $(x, u, y, v)$ be coordinates for $T\left(T_{\partial} X\right)$ and $(x, y, u, v)$ coordinates for $T_{\partial}(T(X))$. A straightforward computation using the formulas above yields that the map taking $(x, y, u, v)$ to $(x, u, y, v)$ gives an isomorphism between $T\left(T_{\partial} X\right)$ and $T_{\partial}(T(X))$ defined over $K$. By gluing, this extends to arbitrary smooth varieties over $K$. We obtain (see [32, Lemma 5.1):

Lemma 2.1. Let $G$ be a commutative algebraic group over $K$. Then there is a functorial isomorphism between the commutative algebraic groups $T\left(T_{\partial} G\right)$ and $T_{\partial}\left(T(G)\right.$ ) (over the natural projections to $T_{\partial}(G)$ ) which also yields a functorial isomorphism between $L\left(T_{\partial} G\right)$ and $T_{\partial}(L G)$.

Suppose now that $(G, s)$ is a commutative algebraic $D$-group over $K$. Then from $s: G \rightarrow T_{\partial} G$ we obtain (differentiating at the identity and setting $d_{G / K, e}=L$ ) a homomorphism $L s: L G \rightarrow L\left(T_{\partial} G\right)$ which, via the identifications of Lemma 2.1, gives a (regular) homomorphic section $L s: L G \rightarrow T_{\partial}(L G)$, that is, a $D$-group structure on $L G$, all defined over $K$. The corresponding logarithmic derivative on $L G$ is $\partial-L s: L G \rightarrow L L G=L G$, namely $\partial \ell n_{(L G, L s)}$, or $\partial \ell n_{L G}$ when $L s$ is assumed. This is the $D$-module structure on $L G$ that we are interested in, and we will denote it $\partial_{L G}$ for simplicity. $(L G)^{\partial}$ will denote the kernel of $\partial_{L G}$. For a geometric account, see Sections C and D of the Appendix.

Remark 2.2. If $G$ has dimension $n$ and is defined over $C_{K}$ and $s=0$, then $(L G, L s)$ is isomorphic to $\left(\mathbb{G}_{a}^{n}, 0\right)$ and $\partial_{L G}=\partial$ on $\mathbb{G}_{a}^{n}$.

We point out two alternative algebraic descriptions of $\partial_{L G}$. The first is as in [32], Lemma 3.7 (iii) and the paragraph following it: we know that $s: G \rightarrow T_{\partial} G$ gives a derivation, which we still call $s$, of the structure sheaf of $G$ over $K$ extending $\partial \mid K$. Now, $s$ acts on the local ring of $G$ at the identity, and in fact preserves the maximal ideal $\mathcal{M}$. So $s$ induces a $D$-module structure on the cotangent space $\mathcal{M} / \mathcal{M}^{2}$ at identity, and hence the dual connection on $L G$. This can be checked to coincide with $\partial_{L G}$, as mentioned in [32, Lemma 5.1 (ii).

Another description is via differentials of differential regular functions, as defined by Kolchin [21, Section 2 of Chapter 8 . We have the differential regular homomorphism $\partial \ell n_{G}: G \rightarrow L G$, which has a differential $L \partial \ell n_{G}$ at the identity, a differential regular homomorphism from $L G$ to $L G$ which again can be shown to coincide with $\partial_{L G}$. 
2.4. Algebraic $D$-groups and differential algebraic groups. We recall some results and facts from 23. Suppose that $\left(G, s_{G}\right),\left(H, s_{H}\right)$ are connected commutative algebraic $D$-groups defined over $K$ and that $h: G \rightarrow H$ is a homomorphism of algebraic groups defined over $K$. We will say that $h$ is a homomorphism of algebraic $D$-groups, if $T_{\partial} h \circ s_{G}=s_{H} \circ h$.

If $H$ is an algebraic subgroup of the algebraic $D$-group $(G, s)$, we say that $H$ is a $D$-subgroup if $s \mid H: H \rightarrow T_{\partial} H \subseteq T_{\partial} G$. (Likewise for " $D$-subvariety".)

From 2.7 of [23] we obtain (suppressing the mention of $s$ sometimes) the easy

Fact 2.3. (i) Let $h: G \rightarrow H$ be a homomorphism of algebraic $D$-groups. Then $\operatorname{Ker}(h)$ is an algebraic $D$-subgroup of $G$.

(ii) Conversely, if $H$ is an algebraic $D$-subgroup of $G$, then $G / H$ can be equipped with the structure of a $D$-group such that the quotient map is a $D$-homomorphism.

From Fact 2.3 of $[23$ ] we have the deeper

Fact 2.4. (i) If $G$ is an algebraic $D$-group, then $G^{\partial}$ is Zariski-dense in $G$. Consequently, if $\left(G, s_{G}\right),\left(H, s_{H}\right)$ are connected algebraic $D$-groups and $h: G \rightarrow H$ is a homomorphism of algebraic groups, then $h$ is a $D$-homomorphism if and only if $h\left(G^{\partial}\right) \subseteq H^{\partial}$.

(ii) Let $G$ be a connected algebraic $D$-group. Then we have a bijection between (connected) algebraic $D$-subgroups of $G$ and (connected) differential algebraic subgroups of $G^{\partial}$ : namely, for $H$ a $D$-subgroup of $G, G^{\partial} \cap H=H^{\partial}$ is a differential algebraic subgroup of $G^{\partial}$ whose Zariski closure is $H$. Also, if $\mathcal{H}$ is a differential algebraic subgroup of $G^{\partial}$, then the Zariski closure of $\mathcal{H}$ in $G$ is a $D$-subgroup whose intersection with $G^{\partial}$ is precisely $\mathcal{H}$.

We will also need the following easy fact (a converse statement for embeddings is given in Corollary G.5 of the Appendix):

Lemma 2.5. Suppose that $f: G \rightarrow H$ is a homomorphism of algebraic D-groups. Then $L f: L G \rightarrow L H$ is a homomorphism of D-modules (where $L G, L H$ are equipped with D-module structures as in Section 2.3).

Note. Throughout Section 2.4, we can also specify fields of definition where appropriate. For example in Fact 2.3 (i) if $h$ is defined over $K$, so is $\operatorname{Ker}(h)$, and in Fact 2.3 (ii) if $G, H$ are defined over $K$, so is $G / H$.

\section{Almost semi-abelian $D$-groups}

We recall that we work in characteristic 0 , in the context of a universal differential field $\mathcal{U}$ and with a small differential subfield $K$. We maintain our general assumption that $K$ is algebraically closed, but it is not always needed. Although much of what we say is implicit or explicit in the literature (such as [9] and [26]), we may give proofs for the convenience of the reader.

3.1. Almost semi-abelian varieties and $D$-groups. Recall that an abelian variety $A$ has a "universal vectorial extension", which we denote by $\tilde{A}$ throughout the paper, and which admits the following characterization: there is an exact sequence of commutative connected algebraic groups,

$$
0 \longrightarrow W_{A} \longrightarrow \tilde{A} \stackrel{\pi}{\longrightarrow} A \longrightarrow 0,
$$


such that $W_{A}$ is unipotent (i.e., a vector group) and such that for any extension $f: G \rightarrow A$ of $A$ by a vector group there is a unique homomorphism $h: \tilde{A} \rightarrow G$ such that $\pi=f \circ h$.

Moreover, if $A$ is defined over $K$, so is $\tilde{A}$. In fact $W_{A}$ is the dual of $H^{1}\left(A, \mathcal{O}_{A}\right)$ and so $\tilde{A}$ has dimension $2 \operatorname{dim}(A)$. See [28] for a detailed presentation.

Likewise any semi-abelian variety $B$ has a universal vectorial extension $\tilde{B}$ with the same universal property as above. In fact if $B$ is an extension of the abelian variety $A$ by the algebraic torus $T$, then $\tilde{B}$ is $B \times_{A} \tilde{A}$, which is an extension of $B$ by $W_{A}$. Again if $B$ is defined over $K$, so is $\tilde{B}$.

Lemma 3.1. Let $G$ be a commutative connected algebraic group defined over $K$. The following conditions are equivalent:

(i) $G$ has no non-zero homomorphisms to $\mathbb{G}_{a}$;

(ii) there exists a semi-abelian variety $B / K$ and a unipotent subgroup $U / K$ of $\tilde{B}$ such that $G$ is isomorphic to $\tilde{B} / U$;

(iii) the group Tor $(G)$ of torsion points of $G$ is Zariski-dense in $G$.

Proof. (i) $\Rightarrow$ (ii): by Chevalley's theorem, $G$ is an extension of its maximal semiabelian quotient $B$ by a unipotent group, say $V$. Let $h: \tilde{B} \rightarrow G$ be given by the universal properties of $\tilde{B}$. So $U=\operatorname{Ker}(h)$ is unipotent. Let $H=h(\tilde{B})$. So $H$ projects onto $B$, and thus $G / H$ is unipotent (a subgroup of $V$ ). As we are assuming $G$ has no unipotent quotients, $G=H$, and $G=\tilde{B} / U$ as required.

(ii) $\Rightarrow$ (iii): since $\operatorname{Tor}(B)$ is Zariski dense in $B$, and since $\tilde{B}$ is an essential extension of $B, \operatorname{Tor}(\tilde{B})$ too is Zariski dense in $\tilde{B}$, and the same property is satisfied by the quotient $G$ of $\tilde{B}$.

(iii) $\Rightarrow$ (i): suppose that $f$ is a surjective homomorphism from $G$ to $\mathbb{G}_{a}$. Then $\operatorname{Tor}(G)<\operatorname{Ker}(f)$.

Definition 3.2. We will call $G$ almost semi-abelian (asa) if it satisfies the equivalent conditions of Lemma 3.1.

Remark 3.3. Inspired by Brion's concept of anti-affine groups [6], we could alternatively say that $G$ is anti-additive. We refer the reader to the first part of Hypothesis $(\mathbf{H})$ of Section I of the Appendix for a Betti version of Condition (iii) (which we could have phrased in $\ell$-adic terms).

For the rest of this section we consider $D$-structures on asa groups. By an almost semi-abelian $D$-group, we mean an algebraic $D$-group $(G, s)$ such that $G$ is almost semi-abelian.

Lemma 3.4. (i) If $G$ is asa, then $G$ has at most one structure of a D-group. Moreover, if $G$ is defined over $K$, then so is the $D$-structure, if it exists.

(ii) If $B$ is semi-abelian, then $\tilde{B}$ has a (unique) structure of D-group.

(iii) If $B$ is semi-abelian and defined over $K$, then $B$ has the structure of a $D$-group if only if $B$ descends to $C_{K}$.

Proof. (i) If $s_{1}, s_{2}$ were distinct rational homomorphic sections $G \rightarrow T_{\partial} G$, then $s_{1}-s_{2}$ would be a non-zero rational homomorphism from $G$ to its Lie algebra. By condition (i) of Lemma 3.1, this gives the first part. If $(G, s)$ is a $D$-structure on $K$, so is $(G, \sigma(s))$ for any automorphism of the field $\mathcal{U}$ fixing $K$ pointwise. So from uniqueness, $s$ is defined over $K$. 
(ii) Let $\pi: \tilde{B} \rightarrow B$, and $\tau: T_{\partial} \tilde{B} \rightarrow \tilde{B}$. So $\pi \circ \tau: T_{\partial} \tilde{B} \rightarrow B$ has kernel a vector group, and thus there is a rational homomorphism $s: \tilde{B} \rightarrow T_{\partial} \tilde{B}$ such that

$(*)$

$$
\pi \circ \tau \circ s=\pi \text {. }
$$

We claim that $s$ is a section of $\tau$, i.e., that $\tau \circ s=i d$. Otherwise, by $(*), \tau \circ s-i d$ is a non-trivial rational homomorphism from $\tilde{B}$ to $\operatorname{ker}(\pi)$. As the latter is a vector group, this contradicts $\tilde{B}$ being asa.

For part (iii) we simply quote Buium (9], Theorem 3 of the Introduction). When $B$ is an abelian variety $A$ over $\mathbb{C}(S)$, this reflects the fact that by its very definition (cf. Section A of the Appendix), the class in $H^{1}(A, T A)$ of the $T A$-torsor $T_{\partial} A$ is given by the Kodaira-Spencer map $\kappa(\partial)$ whose vanishing amounts to $A$ descending to $\mathbb{C}$.

By virtue of Lemma 3.4 (i), we can and will talk about an almost semi-abelian $D$-group $G$ without explicitly mentioning $s$.

Lemma 3.5. Suppose $G$ is an asa D-group. Then $G^{\partial}$ is the "Kolchin closure" of $\operatorname{Tor}(G)$, namely the smallest differential algebraic subgroup of $G$ containing Tor $(G)$.

Proof. Since the homomorphism $\partial \ell n_{G}$ takes values in a vector group, its kernel $G^{\partial}$ contains $\operatorname{Tor}(G)$. Suppose for contradiction that there is a proper differential algebraic subgroup $\mathcal{H}$ of $G^{\partial}$ containing Tor $(G)$. By Fact 2.4 (ii), the Zariski closure $H$ of $\mathcal{H}$ would be a proper $(D$-)subgroup of $G$, contradicting the Zariski-denseness of $\operatorname{Tor}(G)$ given by Lemma 3.1 (iii).

Corollary 3.6. If $G$ is an asa D-group, then any rational homomorphism $f: G \rightarrow$ $H$ from $G$ to a commutative algebraic $D$-group $(H, s)$ is a $D$-homomorphism. In particular, any rational homomorphism between asa D-groups is a D-homomorphism.

Proof. We use Fact 2.4 (i). By the same argument as above, the kernel $(H, s)^{\partial}$ of $\partial \ell n_{H, s}$ contains Tor $(H)$. Hence $f^{-1}\left((H, s)^{\partial}\right)$, a differential algebraic subgroup of $G$, contains $\operatorname{Tor}(G)$. By the previous lemma, $f^{-1}\left((H, s)^{\partial}\right)$ contains $G^{\partial}$, so $f\left(G^{\partial}\right) \subseteq H^{\partial}$.

Note the special case of Corollary 3.6 when $f$ is an embedding:

Corollary 3.7. If $H$ is an asa D-group and is an algebraic subgroup of the commutative algebraic D-group $(G, s)$, then $H$ (with its unique $D$-group structure) is a $D$-subgroup of $(G, s)$.

Also, Corollary 3.6 together with Lemma 3.1 and Lemma 3.4(ii) yields:

Corollary 3.8. Let $G$ be an asa $D$-group, let $B$ be its maximal semi-abelian quotient, and let $U$ be a unipotent subgroup of $\tilde{B}$ such that the algebraic groups $G$ and $\tilde{B} / U$ are isomorphic. Then, $U$ is a $D$-subgroup of $\tilde{B}$. Note that if $G$ is defined over $K$, so are $\tilde{B}$ and $U$.

Let us note in passing that the class of almost semi-abelian $D$-groups is closed under quotienting by algebraic $D$-subgroups, but not of course under $D$-subgroups. Finally, we clearly obtain from Corollary 3.6 that if $B$ is a semi-abelian variety, then its universal vectorial extension $\tilde{B}$, equipped with its unique $D$-structure, is also universal in the category of $D$-group extensions of $B$ by vector groups: namely, if $(G, s)$ is an algebraic $D$-group, and $G$ is, as an algebraic group, an extension of 
$B$ by a vector group, then there is a unique morphism of $D$-groups from $\tilde{B}$ to $G$ satisfying the appropriate commutative diagram.

The $\sharp$-point functor on algebraic groups.

For the sake of completeness we tie up these notions with the $\sharp$-point functor, which will make a brief appearance in Section 6 of the paper. Note that here we do not require $D$-group structures. For $G$ an arbitrary connected commutative algebraic group (over $\mathcal{U}$ ), we say that " $G^{\sharp}$ exists" if the intersection of all the Zariski-dense differential algebraic subgroups of $G$ is still Zariski dense in $G$, and we then denote it by $G^{\sharp}$. Recall that if $G$ is almost semi-abelian (not necessarily a $D$-group) and if $B$ denotes the semi-abelian "part" of $G$, then we have a canonical surjective homomorphism of algebraic groups $\pi: \tilde{B} \rightarrow G$, and moreover $\tilde{B}$ has a canonical $D$-group structure.

Proposition 3.9. Suppose that the algebraic group $G$ is almost semi-abelian.

(i) Then $G^{\sharp}$ exists and is the Kolchin closure of Tor $(G)$. It also equals $\pi\left(\tilde{B}^{\partial}\right)$.

(ii) Moreover, let $U_{1}$ be the maximal D-subgroup of $\tilde{B}$ contained in the (unipotent) subgroup $W:=K \operatorname{Ker}(\pi)$. Then $\pi$ induces an isomorphism of differential algebraic groups between $\left(\tilde{B} / U_{1}\right)^{\partial}$ and $G^{\sharp}$.

Proof. (i) By Lemma 4.2 of [30], which is due originally to Buium, any Zariski-dense differential algebraic subgroup of $G$ must contain $\operatorname{Tor}(G)$, but $\operatorname{Tor}(G)$ is Zariskidense. As by Lemma 3.5, $\tilde{B}^{\partial}$ is the Kolchin closure of $\operatorname{Tor}(\tilde{B})$ and $\pi$ takes $\operatorname{Tor}(\tilde{B})$ onto $\operatorname{Tor}(G)$; it is easy to conclude the rest of part (i).

Notice that $G^{\sharp}=G^{\partial}$ when we further assume that $G$ is a $D$-group. However, in the general case under study here, the differential algebraic group $G^{\sharp}$ need not be defined by first order equations.

(ii) $W \cap \tilde{B}^{\partial}$ is a (connected) differential algebraic subgroup of $\tilde{B}^{\partial}$, so by Fact 2.4 (ii) its Zariski closure is a (connected, unipotent) $D$-subgroup $U_{1}$ of $\tilde{B}$, and $U_{1}^{\partial}$ is precisely $W \cap \tilde{B}^{\partial}$. Then (by the surjectivity of $\left.\partial \ell n_{U_{1}}\right)\left(\tilde{B} / U_{1}\right)^{\partial}$ is canonically isomorphic to $\tilde{B}^{\partial} / U_{1}^{\partial}=\tilde{B}^{\partial} / W \cap \tilde{B}^{\partial}$, which is isomorphic to $G^{\sharp}$ under $\pi$.

Note in particular that if $B$ is a semi-abelian variety, then $B^{\sharp}$ is canonically isomorphic to $\left(\tilde{B} / U_{B}\right)^{\partial}$, where $U_{B}$ is the maximal unipotent $D$-subgroup of $\tilde{B}$. For instance, consider the case where $B=A$ is a simple abelian variety defined over $K$, which does not descend to $C_{K}$ (equivalently, by simplicity, whose $C_{K}$-trace is 0 ), and let $U_{A}$ be the maximal unipotent $D$-subgroup of $\tilde{A}$; so, $U_{A}$ is contained in the kernel $W_{A}$ of $\tilde{A} \rightarrow A$. Since $A$ is not constant, Lemma 3.4 (iii) shows that $U_{A}$ is strictly contained in $W_{A}$. However, $U_{A}$ need not vanish, as witnessed by the following example, which was shown to us by Y. André: take a non-constant type IV abelian variety $A$ of even dimension $g=2 k \geq 4$, such that $\mathbb{Q} \otimes \operatorname{End}(A)$ is a CM field of degree $g$, acting on $L \tilde{A}$ by a CM type of the form $\left\{r_{1}=s_{1}=1, r_{2}=\ldots=\right.$ $\left.r_{k}=2, s_{2}=\ldots=s_{k}=0\right\}$. Then, the $g$-dimensional $K$-vector space $L W_{A} \simeq W_{A}$ is generated by two lines in $L \tilde{A}$ respectively contained in the planes where $F$ acts via the complex embedding $\sigma_{1}, \bar{\sigma}_{1}$, and by the planes where it acts via $\sigma_{2}, \ldots, \sigma_{k}$, whereas $U_{A}$ is generated only by the latter planes, and therefore has dimension $g-2>0$ over $K$.

Remark 3.10. If $B$ is a semi-abelian variety over $K$, then it is convenient to have some notation for the algebraic $D$-group $\tilde{B} / U_{B}$. Let us call it $\bar{B}$. With this notation, Proposition 3.9 (ii) gives a canonical isomorphism (over $K$ ) between $\bar{B}^{\partial}$ and $B^{\sharp}$. 
The reader might think it natural to restrict our attention in Theorem 1.3 to $D$ groups of the the form $\bar{B}$. However, our proofs will be of an inductive nature and involve taking $D$-quotients. Just as with the discussion about $\tilde{B}$ after Remark 1.2, this will of necessity force us into the general class of asa $D$-groups.

3.2. Isoconstant $D$-groups. One usually says that an algebraic group $G / K$ is isoconstant if there is an algebraic group $H / C_{K}$ such that $G$ and $H$ become isomorphic over an extension of $K$. Since $K$ is algebraically closed, they then are automatically isomorphic over $K$.

Definition 3.11. Let $(G, s)$ be a commutative algebraic $D$-group defined over the algebraically closed differential field $K$. We will say that $\left(G, s_{G}\right)$ is isoconstant if there is an algebraic group $\left(H, s_{H}\right)$ such that $H$ is defined over $\mathcal{C}$ and $s_{H}=0$, and there is an isomorphism $f$ (over $\mathcal{U}$ ) of algebraic $D$-groups between $\left(G, s_{G}\right)$ and $\left(H, s_{H}\right)$.

So, the prefix "iso" here refers to differential, rather than algebraic, extensions of the (algebraically closed) base $K$. Once isoconstancy holds, we can actually insist that the data $\left(H, s_{H}\right)$ and the isomorphism $f$ of this definition be defined over $K^{\text {diff }}$, whereby (as $C_{K}=C_{K^{\text {diff }}}$ ) $H$ will be over $C_{K}$. But again, the isomorphism need not be defined over $K$, as is shown by considering $D$-modules (cf. Remark 3.14 below). However, for asa $D$-groups, we do have rigidity:

Lemma 3.12. Suppose $G$ is an asa D-group defined over the algebraically closed field $K$, and suppose that $G$ is isoconstant. Then $G$ is isomorphic over $K$ to a constant D-group $(H, 0)$.

Proof. By isoconstancy, and because $K$ is algebraically closed, the algebraic group $G$ is isomorphic over $K$ to an algebraic group $H$ defined over $C_{K}$. But then $H$ is $a s a$, and by Corollary 3.6 this isomorphism is also one of $D$-groups.

If $(G, s)$ is an algebraic $D$-group, it is not hard to see that it has a unique maximal connected isoconstant $D$-subgroup. For the next lemma we need to know that the image of any isoconstant algebraic $D$-group $(G, s)$ under a $D$-homomorphism $f$ is also isoconstant. This can be seen in various ways, one of which is as follows: we may assume $G$ defined over $\mathcal{C}$ and $s=0$. By Fact $2.3(\mathrm{i}), \operatorname{Ker}(f)$ is a $D$-subgroup of $G$, but $(\operatorname{Ker}(f))^{\partial}$ is clearly a subgroup of $G(\mathcal{C})$ and by Fact 2.4 (i) is Zariski-dense in $\operatorname{Ker}(f)$. Hence $\operatorname{Ker}(f)$ is defined over $\mathcal{C}$ and it is easy to conclude the argument.

Lemma 3.13. Let $G$ be an almost semi-abelian D-group defined over the algebraically closed field $K$. Let $S$ be the maximal isoconstant connected D-subgroup of $G$, let $A$ be the abelian "part" of $G$, and let $U_{A}$ be the maximal unipotent D-subgroup of $\tilde{A}$. Then

(i) $G / S$ has no toric part and is therefore a quotient of $\tilde{A}$.

(ii) If $S=\{0\}$, then $A$ is an abelian variety with $C_{K}$-trace 0 , and $G=\tilde{A} / U_{A}$. $K$.

(iii) Moreover, all the objects (S, $\tilde{A}$, etc.) and isomorphisms are defined over

Proof. (i) The toric part of $G$ is by Corollary 3.7 a $D$-subgroup so it is contained in $S$.

(ii) First of all $G$ has no toric part, so it is a quotient of $\tilde{A}$ by a unipotent $D$-subgroup $U$. As any commutative unipotent $D$-group is isoconstant, our assumption on $G$ forces $U$ to be the maximal unipotent $D$-subgroup of $\tilde{A}$. Now, we 
may work up to isogeny and assume $A$ to be of the form $A_{0} \times A_{1}$, where $A_{0}$ is over $C_{K}$ and $A_{1}$ has $C_{K}$-trace 0 . Then $\tilde{A}=\tilde{A}_{0} \times \tilde{A}_{1}$. But $\tilde{A}_{0}$ is a constant $D$-group, as is its image in $G$ (by the paragraph preceding this lemma). So $A_{0}=0$, and we see that $G$ must be the quotient of $\tilde{A}_{1}$ by its maximal unipotent $D$-subgroup, as required.

(iii) $S$ is defined over $K$ by uniqueness. The rest is clear.

Remark 3.14. Let $(G, s)$ be a (commutative) unipotent $D$-group over $K$. Then $(G, s)$ is isoconstant.

Proof. This of course belongs to the theory of linear differential equations. Identifying $G$ with $\mathbb{G}_{a}^{n}, s$ has the form $x \mapsto(x, A x)$ for $A$ an $n \times n$ matrix over $K$. We can find a basis $\left\{v_{1}, \ldots, v_{n}\right\}$ of $G$ (as a vector space over $\mathcal{U}$ ) which is simultaneously a basis of the $\mathcal{C}$-vector space $(G, s)^{\partial}$. Now, $v_{1}, \ldots, v_{n}$ can be chosen from $K^{\text {diff }}$ (but not always from $K$ ) and can generate the Picard-Vessiot extension for the linear differential system $\partial(-)=A(-)$. With respect to the basis $\left\{v_{1}, \ldots, v_{n}\right\}, s$ becomes 0 , and so over $K\left(v_{1}, \ldots, v_{n}\right),(G, s)$ is isomorphic to $\left(\mathbb{G}_{a}^{n}, 0\right)$.

\section{FURTHER INGREDIENTS AND SPECIAL CASES}

Here we present the key ingredients for the proof of Theorem 1.3.

4.1. The socle theorem. Proposition 4.1 below follows from Corollary 3.11 of 32 (also appearing in the language of $D$-groups as Corollary 2.11 in [23]). The latter generalizes and has its origin in what is often called the "socle theorem" of Hrushovski. This "socle theorem" is actually a combination of Proposition 4.3 of [17, with the validity of the Zilber dichotomy in the theory of differentially closed fields of characteristic 0 . In fact in the case at hand, what is needed can probably be extracted from Hrushovski's results. In any case the relevant statement concerns commutative connected finite-dimensional differential algebraic groups or equivalently commutative, connected groups of finite Morley rank $\mathcal{B}$ definable in the differentially closed field $\mathcal{U}$. By the algebraic socle, as $(\mathcal{B})$, of $\mathcal{B}$ we mean the maximal connected definable subgroup of $\mathcal{B}$ which is definably isomorphic to a group of the form $C(\mathcal{C})$ for $C$ some algebraic group over $\mathcal{C}$. If $X$ is a differential algebraic subset of $\mathcal{B}$, its stabilizer $\operatorname{Stab}_{\mathcal{B}}(X)$ is defined to be $\{g \in \mathcal{B}: g+X=X\}$. The result says that

(*) if $X$ is an irreducible differential algebraic subset of $\mathcal{B}$ such that $\operatorname{Stab}_{\mathcal{B}}(X)$ is finite, then some translate of $X$ is contained in $\operatorname{as}(\mathcal{B})$.

It should be mentioned that this result is quite powerful, and together with the kind of material in Section 3 quickly yields a proof of the function field Mordell-Lang conjecture in characteristic 0 . Now if $\mathcal{B}=G^{\partial}$ for $G$ an algebraic $D$-group, then it follows from Fact 2.4 (ii) that $\operatorname{as}(\mathcal{B})=H^{\partial}$, where $H$ is the maximal connected isoconstant $D$-subgroup of $G$ as introduced before Lemma 3.13. Bearing this in mind, Proposition 4.1 below is simply a principal homogeneous space version of the socle theorem $(*)$ above.

Proposition 4.1. Let $G$ be an almost semi-abelian $D$ group over $K$. Let $Y \subset G$ be a translate (coset) of $G^{\partial}$ and $Z$ be an irreducible differential algebraic subset of $Y$. Let $\mathcal{S}<G^{\partial}$ be the stabilizer of $Z$ (with respect to the action of $G^{\partial}$ on $Y$ ). Suppose that $\mathcal{S}$ is finite. Then $Z$ is contained in a coset $Z^{\prime}$ of $H^{\partial}$, where $H$ is the maximal isoconstant D-subgroup of $G$. Moreover, if $Y, Z$ are defined over $K$, so is $Z^{\prime}$. 
4.2. On the semi-constant part and descent of $(\mathbf{H X})_{K} \cdot K$ will again denote an algebraically closed differential field. Suppose $B$ is a semi-abelian variety over $K$, with $0 \longrightarrow T \longrightarrow B \stackrel{\pi}{\longrightarrow} A \longrightarrow 0$ the canonical short exact sequence. Let $A_{0}<A$ be the $C_{K}$-trace of $A$ (the maximal abelian subvariety of $A$ isomorphic to an abelian variety over $C_{K}$ ) and let $B_{0}=\pi^{-1}\left(A_{0}\right)$. As in the Introduction, we call $B_{0}$ the semiconstant part of $B$. More generally, suppose $G$ is an almost semi-abelian variety with semi-abelian part $B$; in particular, $G=\tilde{B} / U$ for some unipotent subgroup $U$ of $\tilde{B}$. We denote $B$ by $G^{s a}$ and define the semi-constant part of $G$ to be $G_{0}^{s a}$, namely $B_{0}$. In this setting, our hypothesis $(\mathbf{H G})_{0}$ on the almost semi-abelian group $G$ can be stated as follows:

$(\mathbf{H G})_{0}$, the semi-constant part $B_{0}=G_{0}^{s a}$ of $G$, is an isoconstant algebraic group. In other words, the semi-abelian variety $G_{0}^{s a}$ is isomorphic (and then, automatically over $K$ ) to an algebraic group defined over $C_{K}$. It is clear that this hypothesis $(\mathbf{H G})_{0}$ is preserved under quotients.

We will describe concretely $(L G)^{\partial}(K)$ under the assumption $(\mathbf{H G})_{0}$ and conclude that the hypothesis $(\mathbf{H X})_{K}$ is then preserved under quotients.

From now on, we assume that the algebraically closed differential field $K$ has transcendence degree 1 over its field $\mathbb{C}$ of constants. We will make use of two classical results from Deligne's Hodge II 15, which we now explain. Let $A$ be an abelian variety over $K$, and let $\tilde{A}$ be its universal vectorial extension, equipped with its unique $D$-group structure. From Section 2.3 this provides $L \tilde{A}$ with a connection $\partial_{L \tilde{A}}$. In Section $H$ of the Appendix, we check that this connection is the dual of the Gauss-Manin connection on $H_{d R}^{1}(A / K)$. Write $A$ up to isogeny as $A_{0} \times A_{1}$, where $A_{0}$ descends to $\mathbb{C}$ and $A_{1}$ as $\mathbb{C}$-trace 0 . Then $\tilde{A}=\tilde{A}_{0} \times \tilde{A}_{1}$. We will assume that $A_{0}$ is already over $\mathbb{C}$, thus so is $\tilde{A}_{0}$. As the $D$-group structure on $\tilde{A}_{0}$ is trivial, so is the corresponding connection on $L \tilde{A}_{0}$. Consequently, $\left(L \tilde{A}_{0}\right)^{\partial}=L \tilde{A}_{0}(\mathbb{C})$. The above mentioned results translate in the present setting as follows (see Corollary H.5 of the Appendix):

(i) (Semi-Simplicity.) The $D$-module $L \tilde{A}$ is semi-simple, i.e., a direct sum of simple $D$-modules over the algebraically closed field $K$ (Deligne; cf. 15, II, 4.2.6).

(II) (Theorem of the fixed part.) A horizontal vector of $\partial_{L \tilde{A}}$ which is invariant under the monodromy lies in $L \tilde{A}_{0}$ (Griffiths-Schmid-Deligne; cf. [15, II, 4.1.2). In other words, $(L \tilde{A})^{\partial}(K)=L \tilde{A}_{0}(\mathbb{C})$.

Lemma 4.2. (i) Let $B$ be a semi-abelian variety over $K$. Assume that its semiconstant part $B_{0}$ is defined over $\mathbb{C}$, and let $\tilde{B}_{0} \subseteq \tilde{B}$ be the universal vectorial extension of $B_{0}$. Let $U$ be a unipotent $D$-subgroup of $\tilde{B}$, let $G$ be the asa D-group $\tilde{B} / U$, and let $U_{0}=\tilde{B}_{0} \cap U$. Then, $(L G)^{\partial}(K)=L\left(\tilde{B}_{0} / U_{0}\right)(\mathbb{C})$.

(ii) Let $G$ be an asa D-group over $K$ satisfying $(\mathbf{H G})_{0}$. Let $x \in L G(K)$ be such that for no proper algebraic subgroup $H$ of $G$ over $K$ is $x \in L H(K)+(L G)^{\partial}(K)$. Let $G^{\prime}$ be a quotient of $G$ by a $D$-subgroup defined over $K$, so it is itself an asa D-group. Let $x^{\prime} \in L G^{\prime}(K)$ be the image of $x$ under the corresponding projection $L G \rightarrow L G^{\prime}$. Then for no proper algebraic subgroup $H^{\prime}$ of $G^{\prime}$ over $K$ is $x^{\prime} \in L H^{\prime}(K)+\left(L G^{\prime}\right)^{\partial}(K)$.

Proof. (i) Note first that $U_{0}$ is a $D$-subgroup of the constant $D$-group $\tilde{B}_{0}$, so it is also defined over the constants and has trivial $D$-group structure. Hence $\left(L\left(\tilde{B}_{0} / U_{0}\right)\right)^{\partial}$ is the set of constant points of $L\left(\tilde{B}_{0} / U_{0}\right)$. Let $T$ and $A=A_{0} \times A_{1}$ be as usual for $B$. Identifying $U$ with a unipotent $D$-subgroup of $\tilde{A}$, we have the exact sequence

$$
0 \rightarrow L T(\mathbb{C}) \rightarrow(L G)^{\partial}\left(K^{\text {diff }}\right) \rightarrow(L(\tilde{A} / U))^{\partial}\left(K^{\text {diff }}\right) \rightarrow 0 .
$$


Let $x \in(L G)^{\partial}(K)$, and let $\bar{x}$ be its image in $(L(\tilde{A} / U))^{\partial}(K)$. By (I) above, $\bar{x}$ lifts to a point $\bar{\xi} \in(L \tilde{A})^{\partial}(K)$. By (II) above the latter space is precisely $\left(L \tilde{A}_{0}\right)(\mathbb{C})$. Hence $\bar{x} \in L\left(\tilde{A}_{0} / U_{0}\right)(\mathbb{C})$. This now implies that $\bar{x}$ lifts to a point $\xi \in L\left(\tilde{B}_{0} / U_{0}\right)(\mathbb{C})$. But $x, \xi \in(L G)^{\partial}(K)$ and have the same image in $(L(\tilde{A} / U))^{\partial}$. Hence their difference lies in $L T(\mathbb{C})$, which is contained in $L\left(\tilde{B}_{0} / U_{0}\right)(\mathbb{C})$. Thus, already $x \in L\left(\tilde{B}_{0} / U_{0}\right)(\mathbb{C})$, and (i) is proved.

(ii) Let $G=\tilde{B} / U$ and $G^{\prime}=\tilde{B}^{\prime} / U^{\prime}$ (with $B, B^{\prime}$ semi-abelian and $U, U^{\prime}$ unipotent $D$-subgroups of $\tilde{B}, \tilde{B}^{\prime}$, respectively). The hypothesis $(\mathbf{H G})_{0}$ says that the semiconstant part $B_{0}$ of $B$ is isoconstant. As $B^{\prime}$ is a quotient of $B$, the semi-constant part $B_{0}^{\prime}$ of $B^{\prime}$ is also isoconstant. Assume, as we may, that $B_{0}$ is already defined over $\mathbb{C}$, and set $U_{0}=\tilde{B}_{0} \cap U, U_{0}^{\prime}=\tilde{B}_{0}^{\prime} \cap U^{\prime}$. It is clear that under the relevant quotient map $f: G \rightarrow G^{\prime}, \tilde{B}_{0} / U_{0}$ maps onto $\tilde{B}_{0}^{\prime} / U_{0}^{\prime}$. As both the latter groups are defined over $\mathbb{C}$, we see that $f$ maps $\left(\tilde{B}_{0} / U_{0}\right)(\mathbb{C})$ onto $\left(\tilde{B}_{0}^{\prime} / U_{0}^{\prime}\right)(\mathbb{C})$. By part (i) of this lemma, it follows that $f$ maps $(L G)^{\partial}(K)$ onto $\left(L G^{\prime}\right)^{\partial}(K)$.

Now let $x, x^{\prime}$ be as in the statement of part (ii) of the lemma, and suppose towards a contradiction that $x^{\prime}=u^{\prime}+z^{\prime}$ with $u^{\prime} \in L H^{\prime}(K)$ for some proper algebraic subgroup $H^{\prime}$ of $G^{\prime}$ defined over $K$ and that $z^{\prime} \in\left(L G^{\prime}\right)^{\partial}(K)$. By what we have just observed, $z^{\prime}$ lifts to a point $z \in(L G)^{\partial}(K)$. Then $x-z \in L G(K)$ and $f(x-z)=u^{\prime} \in L H^{\prime}(K)$. Hence $x-z \in L H(K)$, where $H:=f^{-1}\left(H^{\prime}\right)$ is a proper algebraic subgroup of $G$ defined over $K$, contradicting our hypothesis on $x$. Lemma 4.2 is proved.

4.3. Special cases: Ax and the theorem of the kernel. We give two special cases where Theorem 1.3 holds (and where $(\mathbf{H G})_{0}$ is not mentioned but automatically holds). The first is a special case of Ax's theorem, as slightly generalized in [4, although there are other direct proofs (see, e.g. [19]). Again $K$ is an algebraically closed differential field of transcendence degree 1 over its field $\mathbb{C}$ of constants.

Proposition 4.3. Suppose that $G$ is an isoconstant asa D-group over $K, x \in$ $L G(K)$, and $y \in G(\mathcal{U})$ such that $\partial \ln _{G}(y)=\partial_{L G}(x)$. Assume that $x \notin L H(K)+$ $(L G)^{\partial}(K)$ for any proper algebraic subgroup of $G$ defined over $K$. Then

$$
\operatorname{tr} \cdot \operatorname{deg}(K(y) / K)=\operatorname{dim}(G) \text {. }
$$

Proof. By Lemma 3.12 we may assume that $G$ is defined over $\mathbb{C}$, in which case $\partial \ell n_{G}$ is Kolchin's logarithmic derivative, $\partial_{L G}$ is just $\partial: L G \rightarrow L G$, and so $(L G)^{\partial}\left(K^{\text {diff } f}\right)$ $=L G(\mathbb{C})$. The semi-abelian part $B$ of $G$ is defined over $\mathbb{C}$, and $G=\tilde{B} / U$ for some unipotent subgroup $U$ of $\tilde{B}$ over $\mathbb{C}$

We may assume that $y$ lies in $G\left(K^{\text {diff }}\right)$. Let $\tilde{x} \in L \tilde{B}(K)$ be a lift of $x$, let $\tilde{y} \in \tilde{B}\left(K^{\text {diff }}\right)$ be a solution of $\partial \ell n_{\tilde{B}}(-)=\partial_{L \tilde{B}} \tilde{x}$ and let $\bar{y}$ be the projection of $y$ to $B$. We first check that the relative hull $B_{\bar{y}}$ of $\bar{y}$ fills up $B$, i.e., that there is no proper algebraic subgroup $\bar{H}$ of $B$ (over $\mathbb{C}$ ) such that $\bar{y} \in \bar{H}(\mathcal{U})+B(\mathbb{C})$. Otherwise, the projection $\bar{x}$ of $x$ to $L B(K)$ would satisfy $\partial_{L B}(\bar{x})=\partial \ln _{B}(\bar{y}) \in L \bar{H}(\mathcal{U})$, so $\bar{x} \in(L \bar{H}(\mathcal{U})+L B(\mathbb{C})) \cap L B(K)=L \bar{H}(K)+L B(\mathbb{C})$, and if $H$ denotes the inverse image of $\bar{H}$ in $G, x$ would lie in $L H(K)+L G(\mathbb{C})$, contradicting $(\mathbf{H X})_{K}$.

Now by Proposition 1b of [4], $\operatorname{tr} \cdot \operatorname{deg}(K(\tilde{y}) / K)=\operatorname{dim}(\tilde{B})$. Let $y^{\prime}$ be the image of $\tilde{y}$ under the canonical projection from $\tilde{B}$ to $G$. Then, $\operatorname{tr} \cdot \operatorname{deg}\left(K\left(y^{\prime}\right) / K\right)=\operatorname{dim}(G)$. Now both $y, y^{\prime}$ are in $G\left(K^{\text {diff }}\right)$, and $\partial \ell n_{G}(y)=\partial \ell n_{G}\left(y^{\prime}\right)=\partial_{L G} x$. Hence $y-y^{\prime} \in$ $\operatorname{Ker}\left(\partial \ell n_{G}\right)\left(K^{\operatorname{diff}}\right)=G(\mathbb{C})$. So $\operatorname{tr} \cdot \operatorname{deg}(K(y) / K)=\operatorname{dim}(G)$, as required. 
The next special case is fundamental and depends on Manin's theorem of the kernel, in a stronger form due to Chai [12. A direct proof of this result is given in Section K of the Appendix (see Remark K.2 for a discussion of Chai's full sharpening). We take $G$ to be an almost abelian $D$-group over $K$; namely, for some abelian variety $A$ over $K, G$ is a quotient of $\tilde{A}$ by some unipotent $D$-subgroup $V$. As usual, we have $0 \rightarrow W_{A} \rightarrow \tilde{A} \rightarrow A \rightarrow 0$ over $K$, and we denote by $U_{A}$ the maximal unipotent $D$-subgroup of $W_{A}$. So, $G=\tilde{A} / V$, with $V \subset U_{A}$. It follows that $W_{A} / V$ is the unipotent part of $G$, and we write it as $W_{G}$. $W_{G}$ can be identified with its Lie algebra $L W_{G}$, which is itself contained in $L G$.

With the above notation, Chai's theorem reads as follows (cf. Theorem K.1):

Proposition 4.4. Suppose $A$ has $\mathbb{C}$-trace 0 . Suppose that $x \in L G(K)$ and $y \in$ $G(K)$ are such that $\partial \ln _{G}(y)=\partial_{L G}(x)$. Then $x \in L W_{G}(K)$.

Notice that conversely, given $x \in L W_{G}(K)$, there does exist a point $y \in G(K)$ such that $\partial \ell n_{G}(y)=\partial_{L G}(x)$, namely $y=x$ itself, viewed as a point in $W_{G} \subset G$. Indeed, Corollary G. 4 of the Appendix shows that $\partial \ell n_{G}$ and $\partial_{L G}$ coincide on $W_{G} \simeq$ $L W_{G}$.

\section{Proofs of the MAIN Results}

Throughout this section $K$ is an algebraically closed differential field of transcendence degree 1 over its field of constants which is assumed to be $\mathbb{C}$.

5.1. Proof of Theorem 1.3. We recall that $G$ is an almost semi-abelian $D$-group over $K$ satisfying $(\mathbf{H G})_{0}$ (its semi-constant part is constant). We take $x \in L G(K)$ satisfying $(\mathbf{H X})_{K}: x \notin L(H)(K)+(L G)^{\partial}(K)$ for any proper algebraic subgroup $H$ of $G$ over $K$. Given any $y \in G(\mathcal{U})$ satisfying $\partial \ln _{G}(y)=\partial_{L G}(x)$, we must prove that $\operatorname{tr} \cdot \operatorname{deg}(K(y) / K)=\operatorname{dim}(G)$.

Our proof is of a differential algebraic nature. We work in $\mathcal{U}$; that is, we identify (differential) algebraic varieties and groups with their pointsets in $\mathcal{U}$. We first recall the notion of "generic points" of differential algebraic varieties. For $X$ an irreducible finite-dimensional differential algebraic variety, defined over $K$, by a generic point of $X$ over $K$ we mean a point $\alpha \in X$ such that $\operatorname{tr} \cdot \operatorname{deg}(K\langle\alpha\rangle / K)$ is maximum possible. If $X$ is of the form $(G, s)^{\partial}$ for some algebraic $D$-group $(G, s)$ defined over $K$, then for $\alpha \in G^{\partial}, K\langle\alpha\rangle=K(\alpha)$, and clearly the maximum possible tr.deg$\left(K(\alpha) / K\right.$ ) equals $\operatorname{dim}(G)$ (as $G^{\partial}$ is a differential algebraic subvariety of $G$ which is Zariski-dense in $G$ ). Likewise, if $X$ is a translate of $G^{\partial}$ inside $G$ and is defined over $K$, then max. $\{\operatorname{tr} \cdot \operatorname{deg}(K\langle\alpha\rangle / K): \alpha \in X\}=\operatorname{dim}(G)$.

We now begin the proof proper.

Let $\operatorname{dim}(G)=n$. Let $a=\partial_{L G}(x) \in L G(K)$, and let $Y \subseteq G$ be the solution set of $\partial \ell n_{G}(-)=a$. So $Y$ is a coset (translate) of $G^{\partial}=K \operatorname{Ker}\left(\partial \ell n_{G}\right)$ in $G$, defined over $K$. By the discussion of genericity above, for generic $y \in Y$ (over $K$ ), tr.deg $(K(y) / K)=$ $n$. Our desired conclusion is that for all $y \in Y$, tr.deg$(K(y) / K)=n$. We claim that this is equivalent to saying that $Y$ has no proper differential algebraic subsets defined over $K$. Let us briefly explain the equivalence. The differential equations defining $Y$ in $G$ give, for all $y \in Y$, a rational control of $\partial y$ in terms of $y$. Hence differential algebraic subvarieties of $Y$ defined over $K$ have the form $Z \cap Y$ for $Z$ algebraic subvarieties of $G$ defined over $K$, and our desired conclusion that $Y$ meets no proper algebraic subvariety of $G$ defined over $K$ is equivalent to $Y$ having 
no differential algebraic subvariety defined over $K$. (This is is course related to a version of Fact 2.4 (ii) for subvarieties rather than algebraic subgroups.)

So we will prove:

Lemma 5.1. $Y$ has no proper irreducible differential algebraic subset defined over $K$. (Or in model-theoretic language the formula " $y \in Y$ " isolates a complete type over $K$.)

Proof. Globally the proof proceeds by induction on $n$. If $n=1$, then $G$ is either $\mathbb{G}_{m}$ or an elliptic curve $E$. In either case (owing to Lemma 3.4 (iii)) $G$ descends to $\mathbb{C}$, and so we finish by Proposition 4.3.

So let us assume $n>1$. We will suppose that $Z$ is a proper irreducible differential algebraic subset of $Y$, defined over $K$, and look for a contradiction. This will be a somewhat involved case analysis, reducing to the special cases discussed in Section 4.3 .

Let $\mathcal{S}:=\left\{g \in G^{\partial}: g+Z=Z\right\}<G^{\partial}$ be the stabilizer of $Z$. Then $\mathcal{S}$ is a differential algebraic subgroup of $G^{\partial}$ defined over $K$. By Fact 2.4 (ii), $\mathcal{S}=S \cap G^{\partial}$, where $S$ is the Zariski closure of $\mathcal{S}$ and is an algebraic $D$-subgroup of $G$ defined over $K$. Note that $S$ is a proper subgroup of $G$, for otherwise $\mathcal{S}=G^{\partial}$ and $Z=Y$.

Case I. $\mathcal{S}$ is infinite.

Let $G^{\prime}$ be $G / S$ (in fact it will be enough to quotient by the connected component of $S$ ). Let $\pi: G \rightarrow G^{\prime}$ be the canonical $K$-rational surjective homomorphism. So $G^{\prime}$ is an almost semi-abelian $D$-group, defined over $K$, with dimension positive, and $<n$ and $\pi$ is a homomorphism of $D$-groups, inducing a surjective $K$-rational homomorphism $L \pi$ from $L G \rightarrow L G^{\prime}$, which is also a homomorphism of $D$-modules by Lemma 2.5. It follows that $\pi(Y)$ is the solution space of the equation $\partial \ell n_{G^{\prime}}(-)=$ $a^{\prime}$, where $a^{\prime}=L \pi(a)$. Note also that $\partial_{L G^{\prime}}\left(x^{\prime}\right)=a^{\prime}$, where $x^{\prime}=L \pi(x) \in L G^{\prime}(K)$. By Lemma 4.2 the hypothesis $(\mathbf{H X})_{K}$ holds for $x^{\prime}$. By the induction hypothesis, $\pi(Y)$ has no proper differential algebraic subset defined over $K$. As $\pi(Z)$ is a differential constructible subset of $\pi(Y)$, defined over $K$, it follows that $\pi(Z)=$ $\pi(Y)$. This implies that $Y \subseteq Z+S$. As $Z \subseteq Y, Y$ is a principal homogeneous space for $G^{\partial}$ and $\mathcal{S}=S \cap G^{\partial}$, it follows that $Y=Z+\mathcal{S}$. This contradicts $\mathcal{S}$ being the stabilizer of $Z$ and $Z$ being a proper differential algebraic subset of $Y$. So Case I leads to a contradiction.

Case II. $\mathcal{S}$ is finite.

Let $H$ denote the maximal connected isoconstant $D$-subgroup of $G$ (which is defined over $K$ ). By Proposition $4.1, Z$ is contained in a coset (i.e., an orbit) $Z^{\prime}$ of $H^{\partial}$, and $Z^{\prime}$ is defined over $K$. We again have a $K$-rational surjective homomorphism $\pi: G \rightarrow G / H$ of $D$-groups, with $L \pi: L G \rightarrow L(G / H)$ a surjective homomorphism of $D$-modules. Again, $L \pi(x)=x^{\prime} \in L(G / H)(K)$ and $\partial_{L(G / H)}\left(x^{\prime}\right)=a^{\prime}=L \pi(a) \in$ $L(G / H)(K)$. But now $\pi(Z)=y^{\prime}$ is a point in $\pi(Y)=$ solutions of $\partial \ell n_{G / H}(-)=$ $\left.a^{\prime}\right\}$ since $Z \subset H$, and this point is $K$-rational since $\pi(Z)=\pi\left(Z^{\prime}\right)$ and $Z^{\prime}$ is defined over $K$. Moreover, the hypotheses $(\mathbf{H G})_{0}$ and $(\mathbf{H X})_{K}$ remain valid for $G / H$ and $x^{\prime}$ (using Lemma 4.2).

We have three subcases:

(a) $H=G$.

This means that $G$ itself is an isoconstant $D$-group, and we contradict Proposition 4.3 . 
(b) $H$ is a proper non-zero subgroup of $G$.

So $\operatorname{dim}(G / H)$ is both positive and $<n$, and we can use the induction hypothesis. Since tr.deg $\left(K\left(y^{\prime}\right) / K\right)=0$, we have a contradiction.

(c) $H=\{0\}$.

By Lemma 3.13 (ii) $G=\tilde{A} / U_{A}$, where $A$ is an abelian variety over $K$ with $\mathbb{C}$-trace 0 and $U_{A}$ is the maximal unipotent $D$-subgroup of $\tilde{A}$. Moreover, $y=y^{\prime} \in G(K)$ and $x \in L G(K)$ satisfy $\partial \ln _{G}(y)=\partial_{L G}(x)$. By Proposition 4.4, $x \in W_{G}(K)=$ $L W_{G}(K)$, where $W_{G}$ is a proper algebraic subgroup of $G$ defined over $K$. This contradicts the hypothesis $(\mathbf{H X})_{K}$.

We have shown that all cases lead to a contradiction. So Lemma 5.1 is proved, as is Theorem 1.3.

5.2. Dropping $(\mathbf{H G})_{0}$. We give a promised version of Theorem 1.3.

Theorem 5.2. Let $G$ be an asa D-group over $K, x \in L G(K)$ and assume that

(HX): for no proper algebraic subgroup $H$ of $G$ defined over $K$ is $x \in L H\left(K^{\text {diff }}\right)$ $+(L G)^{\partial}\left(K^{\text {diff }}\right)$.

Then for any $y \in G(\mathcal{U})$ such that $\partial \ln _{G}(y)=\partial_{L G}(x)$, $\operatorname{tr} \cdot \operatorname{deg}(K(y) / y)=\operatorname{dim}(G)$.

Proof. So note that there is now no restriction on the semi-constant part of $G$. But the "arithmetic" hypothesis $(\mathbf{H X})_{K}$ on $x$ has been strengthened to the differential algebraic hypothesis HX. The proof is identical to that of Theorem 1.3 above, except that the stronger hypothesis $(\mathbf{H X})$ is easily seen to descend under quotients by $D$-subgroups, while as already noted, there is no $(\mathbf{H G})_{0}$ assumption in the special cases covered by Lemmas 4.3 and 4.4 (in fact it is automatically true there.)

We take the opportunity of this discussion to point out that under either condition $(\mathbf{H X})_{K}$ or $(\mathbf{H X})$, we must consider all proper algebraic subgroups $H$ of $G$, not just its algebraic $D$-subgroups. For instance, if $G=\tilde{A}$ for some non-constant elliptic curve $A / K$, so that $(L G)^{\partial}(K)=0$, and if $0 \neq x \in L W_{A}(K)$, then, $y=x$, viewed as a point of $W_{A} \subset G$, satisfies $\partial \ell n_{G} y=\partial_{L G} x$, in view of Corollary G.4 of the Appendix (see also the remark following Proposition 4.4). We then have tr.deg. $(K(y) / K)=0<\operatorname{dim}(G)$, although since $U_{A}$ here vanishes, $x$ lies in the Lie algebra of no proper $D$-subgroup of $G$.

5.3. A counterexample. We give the simplest possible example showing that in Theorem 1.3, the $(\mathbf{H G})_{0}$ hypothesis cannot be dropped in general. Let $K=$ $\mathbb{C}(z)^{\text {alg }}$, let $E$ be an elliptic curve defined over $\mathbb{C}$ and let $B$ be a non-constant extension of $E$ by $\mathbb{G}_{m}$, defined over $K$ (such extensions are given by $K$-rational points on the dual $\hat{E}$ of $E$, not lying in $\hat{E}(\mathbb{C})$ ). We take as our asa $D$-group the universal vectorial extension $G=\tilde{B}$ of $B$, and recall from Corollary 3.7 (or from Fact H.3 of the Appendix) that $L G$ is an extension of $L \tilde{E}$ by $L \mathbb{G}_{m}$ in the category of $D$-modules over $K$.

Let $\bar{x}$ be a non-zero point in $L E(\mathbb{C})$, which we lift to a point $\tilde{x} \in L \tilde{E}(\mathbb{C})$ and finally to a $K$-rational point $x \in L G(K)$ of $L G$. Then:

Claim I. $G$ does not satisfy $(\mathbf{H G})_{0}$. Indeed, its semi-constant part $G_{0}^{s a}$ is $B$ itself, which is not isoconstant.

Claim II. If $y \in G\left(K^{\text {diff }}\right)$ satisfies $\partial \ln _{G}(y)=\partial_{L G}(x)$, then $\operatorname{tr} \cdot \operatorname{deg}(K(y) / K) \leq 1$. 
Proof. Let $\partial_{L G}(x)=a \in L G(K)$. Then the image of $a$ under the projection to $L \tilde{E}$ is $\partial_{L \tilde{E}}(\tilde{x})=0$. Hence the solution set of $\partial \ell n_{G}(-)=a$ in $G\left(K^{\text {diff }}\right)$ projects onto the solution set of $\partial \ell n_{\tilde{E}}(-)=0$ in $\tilde{E}\left(K^{\text {diff }}\right)$, which is precisely $\tilde{E}(\mathbb{C})$. Since the fibers of the projection $G \rightarrow \tilde{E}$ are one-dimensional, the claim follows.

Claim III. $(L G)^{\partial}(K)=\left(L \mathbb{G}_{m}\right)^{\partial}(K)\left(=L \mathbb{G}_{m}(\mathbb{C})\right)$.

Proof. Since $\mathbb{G}_{m}$ is the maximal constant subgroup of $B$, this follows from the extension to mixed Hodge structures of the theorem of the fixed part, as given in [33, Prop. 4.19.

Claim IV. $x$ satisfies $(\mathbf{H X})_{K}$.

Proof. The only proper algebraic subgroups $H$ of $G$ are $\mathbb{G}_{m}, \mathbb{G}_{a}$ and $\mathbb{G}_{m} \times \mathbb{G}_{a}$. So if $x \in L H(K)+(L G)^{\partial}(K)$, then by Claim III $x \in L\left(\mathbb{G}_{m} \times \mathbb{G}_{a}\right)$, so it could not project to a non-zero element of $L E$. This contradicts the choice of $\bar{x}$.

Claims II and IV show that Theorem 1.3 is in general false without the $(\mathbf{H G})_{0}$ hypothesis. On the other hand, Theorem 5.2 remains valid: since $\tilde{x} \in(L \tilde{E})^{\partial}$, the point $x$ in this example lies in $L \mathbb{G}_{m}\left(K^{\text {diff }}\right)+(L G)^{\partial}\left(K^{\text {diff }}\right)$, and therefore violates hypothesis (HX).

5.4. Extension to several variables. As promised in Remark 1.2 (ii), here we show how Corollary 1.1 implies its own extension to function fields $K^{\prime}$ in several variables. For the sake of clarity, we repeat our notation in this new setting.

Let $S^{\prime}$ be a smooth connected algebraic variety over $\mathbb{C}$, and let $K^{\prime}=\mathbb{C}\left(S^{\prime}\right)$ be its field of rational functions. Let $\pi: \mathbf{B}^{\prime} \rightarrow S^{\prime}$ be a semi-abelian scheme of constant toric rank, with generic fiber $B^{\prime} / K^{\prime}$ and Lie algebra $L B^{\prime} / K^{\prime}$, let $A^{\prime} / K^{\prime}$ be the maximal abelian quotient of $B^{\prime}$, and let $\left(A_{0}^{\prime}, \tau\right)$ be the $K^{\prime} / \mathbb{C}$ trace of $A^{\prime} / K^{\prime}$, where we assume for simplicity that $A_{0}^{\prime}$ is included in $A^{\prime}$ and is equal to the $K^{\prime a l g} / \mathbb{C}$-trace of $A^{\prime} / K^{\prime a l g}$. Further, let $B_{0}^{\prime}$ be the pull-back of $A_{0}^{\prime}$ to $B^{\prime}$, which we again call the semi-constant part of $B^{\prime}$. Finally, let $\tilde{\mathbf{B}}^{\prime}$ be the universal vectorial extension of $\mathbf{B}^{\prime}$, with generic fiber $\tilde{B}^{\prime} / K^{\prime}$. We consider the exponential morphisms of analytic sheaves $\exp _{\tilde{\mathbf{B}}^{\prime}}: L \tilde{\mathbf{B}}^{\prime a n} \longrightarrow \tilde{\mathbf{B}}^{\prime a n}$ over $S^{\prime a n}$ and a point $x^{\prime}$ in $L B^{\prime}\left(K^{\prime}\right)$, extended to $\mathbf{x}^{\prime} \in L \mathbf{B}^{\prime}\left(S^{\prime}\right)$ and lifted to a section $\tilde{\mathbf{x}}^{\prime}$ of $L \tilde{\mathbf{B}}^{\prime}\left(S^{\prime}\right)$, for which we set $\tilde{\mathbf{y}}^{\prime}=\exp _{\tilde{\mathbf{B}}^{\prime}}\left(\tilde{\mathbf{x}}^{\prime}\right) \in$ $\tilde{\mathbf{B}}^{\prime a n}\left(S^{\prime a n}\right)$. Abbreviating this analytic section as $\tilde{y}^{\prime}=\exp _{\tilde{B}^{\prime}}\left(\tilde{x}^{\prime}\right)$ and making use of the same notations over all covers of $S^{\prime}$, we have:

Theorem 5.3. Let $B^{\prime} / K^{\prime a l g}$ be a semi-abelian variety, and let $x^{\prime}$ be a point in $L B^{\prime}\left(K^{\prime a l g}\right)$. Assume that

$\left(\mathbf{H B}_{0}^{\prime}\right)$ : the semi-constant part $B_{0}^{\prime}$ of $B^{\prime}$ is defined over $\mathbb{C}$;

$\left(\mathbf{H X}^{\prime} / \mathbf{B}^{\prime}\right)_{K^{\prime a l g}}$ : for any proper semi-abelian subvariety $H^{\prime}$ of $B^{\prime}, x^{\prime} \notin L H^{\prime}\left(K^{\prime a l g}\right)$ $+L B_{0}^{\prime}(\mathbb{C})$.

Further, let $\tilde{x}^{\prime} \in L \tilde{B}^{\prime}\left(K^{\prime a l g}\right)$ be a lift of $x^{\prime}$. Then, the field of definition of the point $\tilde{y}^{\prime}=\exp _{\tilde{B}^{\prime}}\left(\tilde{x}^{\prime}\right)$ satisfies

$$
\operatorname{tr} . \operatorname{deg} \cdot\left(K^{\prime a l g}\left(\tilde{y}^{\prime}\right) / K^{\prime a l g}\right)=\operatorname{dim}\left(\tilde{B}^{\prime}\right) .
$$

Proof. We will show the existence of an algebraic curve $S$ in $S^{\prime}$, with function field $K=\mathbb{C}(S)$, such that the restrictions $\mathbf{B}=\mathbf{B}_{\mid S}^{\prime}$ of $\tilde{\mathbf{B}}^{\prime}$ and $\mathbf{x}=\mathbf{x}_{\mid S}^{\prime}$ of $\mathbf{x}^{\prime}$ to $S$ satisfy the hypotheses of Corollary 1.1. Since the exponential morphisms commute with 
specialisation, and since transcendence degrees cannot increase under specialization, Corollary 1.1 will then imply that

$$
\operatorname{tr} \cdot \operatorname{deg} \cdot\left(K^{\prime}\left(\tilde{\mathbf{y}}^{\prime}\right) / K^{\prime}\right) \geq \operatorname{tr} \cdot \operatorname{deg} \cdot\left(K\left(\tilde{\mathbf{y}}_{\mid S}^{\prime}\right) / K\right)=\operatorname{dim}\left(\tilde{B}^{\prime}\right)
$$

hence the required equality.

Let $s$ be a point in $S^{\prime}(\mathbb{C})$. The fiber $\mathbf{B}^{\prime}{ }_{s}$ of $\mathbf{B}^{\prime} / S^{\prime}$ is a semi-abelian variety over $\mathbb{C}$ whose maximal abelian quotient $\mathbf{A}_{s}^{\prime}$ is by hypothesis the fiber at $s$ of the abelian scheme $\mathbf{A}^{\prime} / S^{\prime}$. The fiber $\left(\mathbf{B}^{\prime}{ }_{0}\right)_{s}$ of the semi-constant part $\mathbf{B}_{0}^{\prime} / S^{\prime}$ of $\mathbf{B}^{\prime} / S^{\prime}$ is the pull-back to $\mathbf{B}^{\prime}{ }_{s}$ of $A_{0}^{\prime}$, viewed as an abelian subvariety of $\mathbf{A}_{s}^{\prime}$. Because of hypothesis $\left(\mathbf{H B}_{0}^{\prime}\right)$, we may thereby identify $\left(\mathbf{B}_{0}^{\prime}\right)_{s}$ with $B_{0}^{\prime}$. We denote by $\kappa_{s}: T_{s} S^{\prime} \rightarrow H^{1}\left(\mathbf{A}_{s}^{\prime}, T \mathbf{A}_{s}^{\prime}\right)\left(\right.$ resp. $\left.\kappa: \operatorname{Der}\left(K^{\prime} / \mathbb{C}\right) \rightarrow H^{1}\left(A^{\prime} / K^{\prime}, T A^{\prime} / K^{\prime}\right)\right)$ the Kodaira-Spencer map attached to the abelian scheme $\mathbf{A}^{\prime} / S^{\prime}$ at the point $s$ (resp. at the generic point of $S^{\prime}$ ). Similarly, we denote by $d_{s} \mathbf{x}^{\prime}: T_{s} S^{\prime} \rightarrow L \mathbf{B}_{s}^{\prime}$ (resp. $\left.d x^{\prime}: \operatorname{Der}\left(K^{\prime} / \mathbb{C}\right) \rightarrow L B^{\prime} / K^{\prime}\right)$ the differential of $\mathbf{x}^{\prime}: S^{\prime} \rightarrow L \mathbf{B}^{\prime}$ at $s$ (resp. at the generic point of $S^{\prime}$ ). The curve $S$ to be constructed will be attached to a point $\left(\sigma, \partial_{\sigma}\right) \in T S^{\prime}(\mathbb{C})$ chosen in a certain dense open subset $\Omega$ of $T S^{\prime}$, which we now define in three steps.

First, consider the subset $\Omega_{1} \subset S^{\prime}(\mathbb{C})$ formed by the points $s \in S^{\prime}(\mathbb{C})$ satisfying:

- $\Omega_{1}$ : for any semi-abelian subvariety $\mathcal{H} / \mathbb{C}$ of $\mathbf{B}_{s}^{\prime} / \mathbb{C}$, there exists a semi-abelian subscheme $\mathbf{H}^{\prime} / S^{\prime}$ of $\mathbf{B}^{\prime} / S^{\prime}$ such that $\mathbf{H}_{s}^{\prime}=\mathcal{H}$.

By a standard argument (see [1, Lemma 4), the set $\Omega_{1}$ is the complement of a countable union of analytic (and in fact algebraic, by [11]) subvarieties of $S^{\prime a n}$ of positive codimensions. (Recall that $\mathbf{B}_{s}^{\prime} / \mathbb{C}$ has at most countably many semi-abelian subvarieties $\mathcal{H}$.) Notice that by flatness, the extension $\mathbf{H}^{\prime}$ of $\mathcal{H}$ to $S^{\prime}$ is unique.

Second, given our section $\mathbf{x}^{\prime}$ of $L \mathbf{B}^{\prime} / S^{\prime}$, we attach to each proper semi-abelian subscheme $\mathbf{H}^{\prime} / S^{\prime}$ of $\mathbf{B}^{\prime} / S^{\prime}$, with generic fiber $H^{\prime} / K^{\prime}$, a subset $\Delta_{\mathbf{H}^{\prime}}$ of $T S^{\prime}(\mathbb{C})$, defined as follows: fix a decomposition of the $K^{\prime}$-vector space $L B^{\prime}$ as $\left(V_{0}\right)_{K^{\prime}} \oplus V_{1} \oplus$ $V_{2} \oplus V_{3}$, where $V_{1}:=\left(L B_{0}^{\prime}\right)_{K^{\prime}} \cap L H^{\prime}$, while $V_{0}$ is a $\mathbb{C}$-subspace of $L B_{0}$ generating over $K^{\prime}$ a complement of $V_{1}$ in $\left(L B_{0}^{\prime}\right)_{K^{\prime}}, V_{2}$ is a complement of $V_{1}$ in $L H^{\prime}$, and $V_{3}$ is a complement of the sum $\left(L B_{0}^{\prime}\right)_{K^{\prime}}+L H^{\prime}$ of the previous ones in $L B^{\prime}$. Notice that since $H^{\prime} \neq B^{\prime}, V_{0}$ and $V_{3}$ cannot both be $\{0\}$. Withdrawing a hypersurface in $S^{\prime}$, we may assume that these spaces extend to vector bundles over $S^{\prime}$, and for each $s$ in the remaining open part of $S^{\prime}(\mathbb{C})$, we denote by $\mathbf{x}_{i}^{\prime}(s)$ the corresponding components of $\mathbf{x}^{\prime}(s) \in L \mathbf{B}_{s}^{\prime}$. We then set

$$
\Delta_{\mathbf{H}^{\prime}}=\left\{\left(s, \partial_{s}\right) \in T S^{\prime}(\mathbb{C}), \mathbf{x}_{3}^{\prime}(s)=0 \text { and } d_{s} \mathbf{x}_{0}^{\prime}\left(\partial_{s}\right)=0\right\} .
$$

This set is an algebraic subvariety of $T S^{\prime}$, which cannot fill up the (connected) space $T S^{\prime}$; otherwise we would get $d x_{0}^{\prime}\left(\operatorname{Der}\left(K^{\prime} / \mathbb{C}\right)\right)=0$ and $x^{\prime} \in V_{1}\left(K^{\prime}\right)+V_{2}\left(K^{\prime}\right)+V_{0}(\mathbb{C})$ at the generic point of $S^{\prime}$. Hence $x^{\prime} \in L H^{\prime}\left(K^{\prime}\right)+L B_{0}^{\prime}(\mathbb{C})$, contrary to the hypothesis $\left(\mathbf{H X}^{\prime} / \mathbf{B}^{\prime}\right)_{K^{\prime}}$ which $\mathbf{x}^{\prime}$ satisfies. Consequently, the subset of $T S^{\prime}(\mathbb{C})$,

- $\Omega_{0}:=T S^{\prime}(\mathbb{C}) \backslash \bigcup_{\mathbf{H}^{\prime} \neq \mathbf{B}^{\prime}} \Delta_{\mathbf{H}^{\prime}}$,

is the complement of a countable union of algebraic subvarieties of $T S^{\prime}$ of positive codimensions. Indeed, $\mathbf{B}^{\prime}$ has at most countably many semi-abelian subschemes $\mathbf{H}^{\prime}$.

Finally, let $\kappa_{1}, \ldots, \kappa_{h}$ be the components of $\kappa$ corresponding to the various isotypical factors of the abelian scheme $\mathbf{A}^{\prime} / S$. Withdrawing a hypersurface from $S^{\prime}$, we can assume that for all $s \in S^{\prime}(\mathbb{C})$, the rank of $\kappa_{s}$, hence of each of the $\kappa_{i, s}$, 
is maximal (i.e., equal to its generic rank). To each $s$, we then attach the subset $\Omega_{2, s} \subset T_{s} S^{\prime}(\mathbb{C})$ formed by the vectors $\partial_{s} \in T_{s} S^{\prime}(\mathbb{C})$ satisfying:

- $\Omega_{2, s}$ : for each $i=1, \ldots, h$, such that $\kappa_{i} \neq 0$, we have $\kappa_{i, s}\left(\partial_{s}\right) \neq 0$.

This set is the complement of a finite union of linear subspaces of $T_{s} S^{\prime}(\mathbb{C})$, each of positive codimension since the corresponding Kodaira-Spencer map $\kappa_{i}$ does not vanish at $s$. Notice for later use that the indexes $i$ such that $\kappa_{i}=0$ correspond to the isotypical factors of the $K^{\prime} / \mathbb{C}$-trace $A_{0}^{\prime}$ of $A^{\prime}$.

We now define $\Omega$ as the subset of $T S^{\prime}(\mathbb{C})$ formed by the couples $\left(\sigma, \partial_{\sigma}\right) \in \Omega_{0}$ such that $\sigma \in \Omega_{1}$ and $\partial_{\sigma} \in \Omega_{2, \sigma}$. Since $T S^{\prime}(\mathbb{C})$ is a Baire set, $\Omega$ is dense, hence not empty. We fix a point $\left(\sigma, \partial_{\sigma}\right)$ in $\Omega$, as well as an arbitrarily chosen smooth algebraic curve $S \subset S^{\prime}$ passing through $\sigma$ in the direction of $\partial_{\sigma}$. Then the restriction $\mathbf{B}=\mathbf{B}_{\mid S}^{\prime}$ of $\mathbf{B}^{\prime} / S^{\prime}$ to $S$ is a semi-abelian scheme over $S$, and we claim that $\mathbf{B}$ and the restriction $\mathbf{x}=\mathbf{x}_{\mid S}^{\prime}$ of $\mathbf{x}^{\prime}$ to $S$ satisfy the hypotheses of Corollary 1.1. As usual, we write $B$ and $x$ for their values at the generic point of $S$, and we let $K=\mathbb{C}(S)$.

- $\left(\mathbf{H B}_{0}\right): \Omega_{2, \sigma}$ ensures that the Kodaira-Spencer map attached to the restrictions to $S$ of the non-constant factors of $\mathbf{A}^{\prime} / S^{\prime}$ at $\sigma$ do not vanish along $T_{\sigma} S=\mathbb{C} \partial_{\sigma}$. Therefore, the $K / \mathbb{C}$-trace of the maximal abelian quotient $A / K$ of $B / K$ coindices with $A_{0}^{\prime}$, and the semi-constant part $B_{0}$ of $B / K$ coincides with $B_{0}^{\prime}$, which is constant by hypothesis $\left(\mathbf{H B}_{0}^{\prime}\right)$. So, $B / K$ satisfies hypothesis $\left(\mathbf{H B}_{0}\right)$ of Corollary 1.1.

- $(\mathbf{H X} / \mathbf{B})_{K^{a l g}}$ : consider a proper semi-abelian subscheme $\mathbf{H} / S$ of $\mathbf{B} / S$. It specializes at $\sigma$ to a semi-abelian subvariety $\mathcal{H}$ of $\mathbf{B}_{\sigma}=\mathbf{B}_{\sigma}^{\prime}$, which $\Omega_{1}$ ensures to extend to a semi-abelian subscheme $\mathbf{H}^{\prime} / S^{\prime}$ of $\mathbf{B}^{\prime} / S^{\prime}$, necessarily inducing $\mathbf{H}$ over $S$. Assume for a contradiction that $\mathbf{x}(S)$ lay in $L \mathbf{H}(S)+L B_{0}(\mathbb{C})=L \mathbf{H}^{\prime}(S)+$ $L B_{0}^{\prime}(\mathbb{C})$. Specializing this relation at $\sigma$, where $\mathbf{x}(\sigma)=\mathbf{x}^{\prime}(\sigma), d_{\sigma} \mathbf{x}_{0}\left(\partial_{\sigma}\right)=d_{\sigma} \mathbf{x}_{0}^{\prime}\left(\partial_{\sigma}\right)$, we deduce that $\mathbf{x}_{3}(\sigma)$ and $d_{\sigma} \mathbf{x}_{0}\left(\partial_{\sigma}\right)$ would vanish and that $\left(\sigma, \partial_{\sigma}\right)$ would belong to the prohibited set $\Delta_{\mathbf{H}^{\prime}}$. Hence, $x \in L B(K)$ does satisfy hypothesis $(\mathbf{H X} / \mathbf{B})_{K^{a l g}}$ of Corollary 1.1, and this concludes the proof of Theorem 5.3.

\section{6. $K$-LARgeness And a Differential Galois-Theoretic Proof}

We will make an additional assumption, $K$-largeness, on our asa $D$-group $G$ over $K$, and obtain the conclusion of Theorem 1.3, replacing the use of the "socle theorem" (Proposition 4.1) by rather softer tools. At the time of writing we are aware that the $K$-largeness hypothesis is very strong, but nevertheless as such Galois-theoretic methods were the initial motivation of this paper (cf. 4, Remark 1), it seems appropriate to include this material. More to the point, the proof of Theorem 1.3, which these methods provide in this case, is the exact copy of Kolchin's classical proof of the Ostrowski theorem and of its multiplicative analogue (cf. Section 2 of [22]), and this suffices to justify their insertion here.

6.1. $K$-large algebraic $D$-groups. We begin in our general context, where $K$ is an arbitrary (small) algebraically closed differential subfield of $\mathcal{U}$, all in characteristic 0 . For $(G, s)$ an algebraic $D$-group defined over $K$, we say, following [31, that $(G, s)$ is $K$-large if $G^{\partial}\left(K^{\text {diff }}\right)=G^{\partial}(K)$. This makes sense for arbitrary $D$-groups, but we will restrict to the case where $G$ is commutative and use additive notation.

Here are some examples, still with $K$ arbitrary. A constant algebraic $D$-group $(G, s=0)$ is automatically $K$-large (and even $C_{K}$-large). A unipotent $D$-group, although isoconstant, is $K$-large if and only if the corresponding $D$-module is completely solvable over $K$, while if $A$ is an abelian variety over $K$ with $\bar{A}=\tilde{A} / U_{A}$ as in 
Remark 3.10, $T$ is a torus, and $G$ is of the form $T \times \bar{A}$, then $G$ is $K$-large. In other words, the sharp points of an abelian variety $A$ always satisfy $A^{\sharp}\left(K^{\text {diff }}\right)=A^{\sharp}(K)$. For the latter fact, it is enough to consider a simple abelian variety $A$, in which case the statement that $A^{\sharp}\left(K^{\text {diff }}\right)=A^{\sharp}(K)$ is precisely Lemma 2.2 of [27. (which depends on work of Hrushovski and Sokolovic). On the other hand, it follows from Proposition 6.1 below that over $K=\mathbb{C}(z)^{\text {alg }}$, the algebraic $D$-group $G / K$ considered in Section 5.3 is not $K$-large.

We now establish a relationship between the hypothesis $(\mathbf{H G})_{0}$ and $K$-largeness, in a quite general setting, using model-theoretic methods. Recall from Remark 3.10 the notation $\bar{B}$ for $B$ a semi-abelian variety: $\bar{B}$ is the quotient of $\tilde{B}$ by its maximal unipotent $D$-subgroup $U_{B}$.

Proposition 6.1. Let $K$ be an (algebraically closed) differential field. Assume that $C_{K}$ has infinite transcendence degree and that $K$ is the algebraic closure of a differential field which is finitely generated (as a differential field) over $C_{K}$. Let $B$ be a semi-constant semi-abelian variety over $K$, i.e., such that the abelian part of $B$ descends to $C_{K}$. Then the following are equivalent:

(i) $B$ is constant, i.e., descends to $C_{K}$.

(ii) The algebraic D-group $G=\bar{B}$ is K-large.

Proof. (i) implies (ii) is immediate: we may assume that $B$ is defined over $C_{K}$. But then $\tilde{B}$ is defined over $C_{K}$ and its unique $D$-group structure is the trivial one. Hence $U_{B}$ coincides with the maximal unipotent subgroup $W_{B}$ of $\tilde{B}$, and $G:=\tilde{B} / U_{B}=B$ with the trivial $D$-group structure. But then $G^{\partial}\left(K^{\text {diff }}\right)=G^{\partial}\left(C_{K^{\text {diff }}}\right)=G\left(C_{K}\right)=$ $G^{\partial}(K)$.

(ii) implies (i). There is no harm in assuming that both $T$ and $A$ are defined over $C_{K}$ (where $T, A$ are the toric, respectively abelian, parts of $B$ ). We have our exact sequence $0 \rightarrow T \rightarrow \tilde{B} \rightarrow \tilde{A} \rightarrow 0$ of $D$-groups, and note that $\tilde{A}$ is defined over $C_{K}$ and has trivial $D$-structure, while $G=\bar{B}$ is obtained by quotienting $\tilde{B}$ by its maximal unipotent $D$-subgroup $U_{B}$. Note that $U_{B}$ identifies with a unipotent $D$-subgroup of $\tilde{A}$ and is thus defined over $C_{K}$. Then $A^{b}:=\tilde{A} / U_{B}$ is also defined over $C_{K}$, has trivial $D$-structure, and sits in the exact sequence of $D$-groups

$$
0 \rightarrow T \rightarrow G \rightarrow A^{b} \rightarrow 0 .
$$

From $(\dagger)$ we obtain the exact sequence $0 \rightarrow T^{\partial} \rightarrow G^{\partial} \rightarrow\left(A^{b}\right)^{\partial} \rightarrow 0$ of differential algebraic groups, from which, computing points in $K^{\text {diff }}$, we finally derive the exact sequence of groups

$$
0 \rightarrow T\left(C_{K}\right) \rightarrow G^{\partial}\left(K^{\text {diff }}\right) \rightarrow A^{\mathrm{b}}\left(C_{K}\right) \rightarrow 0 .
$$

Let $K_{0}$ be a finitely generated differential field contained in $K$ over which $G$ is defined and such that $K=\left(C_{K} \cdot K_{0}\right)^{\text {alg }}$. We now use the language of "generic points" over $K_{0}$, as discussed at the beginning of Section 5.1.

We will first show that we can find a generic point of $G^{\partial}$ over $K_{0}$ which is also a $K^{\text {diff }}$-rational point. Let $m=\operatorname{dim}(G)$. We will use the exact sequence $(\dagger \dagger)$ above, together with the assumption that $C_{K}$ has infinite transcendence degree and $K_{0}$ is finitely generated. Namely, we first choose a point of $A^{b}$ which is generic over $K_{0}$ and is $C_{K}$-rational. Let $b \in G^{\partial}\left(K^{\text {diff }}\right)$ project to $a$. Now let $c$ be a generic point of $T$ over $K_{0}, a, b$, such that $c$ is $C_{K}$-rational. Finally, let $d$ be the sum of $b$ and c. Then $d \in G^{\partial}\left(K^{\text {diff }}\right)$, and it is straightforward to verify that $\operatorname{tr} \cdot \operatorname{deg}\left(K_{0}(d) / K_{0}\right)$ equals $m$. So $d$ is a generic point of $G^{\partial}$ over $K_{0}$ which is also $K^{d i f f}$-rational. 
As $G$ is $K$-large, $d \in G^{\partial}(K)$, and so $d$ is in the algebraic closure in the modeltheoretic sense of (the finitely generated) $K_{0}$ together with a finite tuple of elements from $C_{K}$. As every element of $G^{\partial}$ is a product of generic elements, it follows that (in $\mathcal{U}) G^{\partial}$ is contained (uniformly) in $\operatorname{acl}\left(\mathcal{C}, K_{0}\right)$ and so is definably isomorphic to the group of $\mathcal{C}$-points of an algebraic group defined over $\mathcal{C}$. This implies (see Fact 2.6 of [23] for example) that $G$ is isomorphic to an algebraic group over $\mathcal{C}$ and hence over $C_{K^{\text {diff }}}=C_{K}$. The same is then true of $B$.

Recalling the notation at the beginning of Section 4.2 and in Remark 3.10, we obtain:

Corollary 6.2. Let $K$ be as in the previous proposition. Let $G$ be an almost semiabelian D-group over $K$. Suppose that it is $K$-large. Then $G_{0}^{\text {sa }}$ descends to $C_{K}$. In other words, (HG) $)_{0}$ holds for $G$.

Proof. Note that $\overline{G_{0}^{s a}}$ is a $D$-subgroup of a $D$-quotient of $G$. Hence it is also $K$-large. Now use Proposition 6.1.

6.2. Differential Galois theory. The point of $K$-largeness is that it allows a Galois theory for equations of the form

$$
\partial \ell n_{G}(-)=a, \quad \text { where } a \in L G(K) .
$$

Namely, suppose $(G, s)$ is a $K$-large algebraic $D$-group and $\alpha \in G\left(K^{\text {diff }}\right)$ is a solution of $(* *)$. Let $F=K(\alpha)$, a differential subfield of $K^{\text {diff }}$, and let $A u t_{\partial}(F / K)$ be the group of automorphisms of the differential field $F$ which fix $K$ pointwise. For $\sigma \in \operatorname{Aut}_{\partial}(F / K), \sigma(\alpha)$ is also a solution of $(* *)$, so $\sigma(\alpha)-\alpha \in \operatorname{Ker}\left(\partial \ell n_{G}\right)=G^{\partial}$ whereby $\sigma(\alpha)=\alpha+\rho_{\sigma}$ for a unique $\rho_{\sigma} \in G^{\partial}\left(K^{\text {diff }}\right)$ and by the $K$-largeness assumption in fact $\rho_{\sigma} \in G^{\partial}(K)$. As pointed out in 31, the map taking $\sigma$ to $\rho_{\sigma}$ establishes an isomorphism between $\operatorname{Aut}_{\partial}(F / K)$ and a differential algebraic subgroup of $G^{\partial}\left(K^{\text {diff }}\right)$, which by Fact 2.4 (ii) is of the form $H^{\partial}\left(K^{\text {diff }}\right)$ for $H$ a $D$ subgroup of $G$ defined over $K$. Moreover there is a Galois correspondence between differential fields in between $K$ and $F$ and $D$-subgroups of $H$ defined over $K$ (or equivalently, by $K$-largeness, over $K^{\text {diff }}$ ).

With notation as above, here are some additional remarks taken from [31, to be used below. Working in $\mathcal{U}$ and noting that $H^{\partial}$ acts on $G$, we see that the orbit of $\alpha$ under $H^{\partial}$ coincides with its orbit under $A u t_{\partial}(\mathcal{U} / \mathcal{C} . K)$ and is a differential algebraic PHS for $H^{\partial}$, defined over $K$. In particular, $\operatorname{tr} \cdot \operatorname{deg}(K(\alpha) / K)=\operatorname{dim}(H)$.

We can now give the promised Galois theoretic proof of Theorem 1.3 in the $K$-large case.

Theorem 6.3. Let $K$ be algebraically closed and of transcendence degree 1 over its field of constants $\mathbb{C}$. Let $G$ be an almost semi-abelian $D$-group which is $K$-large. Let $x \in L G(K)$ and $y \in G(\mathcal{U})$ be such that $\partial \ell_{n_{G}}(y)=\partial_{L G}(x)$. Assume $x$ satisfies $(\mathbf{H X})_{K}$. Then tr.deg $\left.(K(y) / K)\right)=\operatorname{dim}(G)$.

Proof. By Corollary 6.2, $G$ satisfies $(\mathbf{H G})_{0}$, so the theorem follows from Theorem 1.3, but the present proof will avoid the difficult Proposition 4.1. Suppose the conclusion fails, so it fails for some $y$ in $G\left(K^{d i f f}\right)$, for which we assume tr.deg$(K(y) / K)<\operatorname{dim}(G)$. Let the $D$-subgroup $H$ of $G$ be the differential Galois group of $K(y) / K$. As recalled above, $A u t_{\partial}(K(y) / K)$ is isomorphic to $H^{\partial}\left(K^{\text {diff }}\right)$, and since $\operatorname{dim}(H)=\operatorname{tr} \cdot \operatorname{deg}(K(y) / K), H$ is a proper $D$-subgroup of $G$. Let $G^{\prime}=G / H$. The images $y^{\prime}, x^{\prime}$ of $y, x$ under the projections $G \rightarrow G^{\prime}$ and 
$L G \rightarrow L G^{\prime}$ satisfy $\partial \ell n_{G^{\prime}}\left(y^{\prime}\right)=\partial_{L G^{\prime}}\left(x^{\prime}\right)$. Also, by Corollary 6.2 and Lemma 4.2, $(\mathbf{H X})_{K}$ is valid for $x^{\prime}$ and $L G^{\prime}$. But now, as the orbit of $y$ under $H^{\partial}$ was defined over $K, y^{\prime}$ is $K$-rational, i.e., in $G^{\prime}(K)$. Furthermore, for any $D$-quotient $G^{\prime \prime}$ of $G^{\prime}$ defined over $K$, these points $y^{\prime}, x^{\prime}$ project to rational points $y^{\prime \prime}, x^{\prime \prime}$ in $G^{\prime \prime}(K), L G^{\prime \prime}(K)$ still satisfying $\partial \ln _{G^{\prime \prime}}\left(y^{\prime \prime}\right)=\partial_{L G^{\prime \prime}}\left(x^{\prime \prime}\right)$ and $(\mathbf{H X})_{K}$. Hence we may assume that $G^{\prime}$ has no proper connected $D$-subgroups. In particular the maximal connected isoconstant $D$-subgroup of $G^{\prime}$ is either $G^{\prime}$ itself or 0. Proposition 4.3 or 4.4 will then give a contradiction.

\section{Appendix: Exponentials on ALgebraic $D$-GRoups}

By "the text", we mean the main body of the present article.

\section{A. Setting.}

Let $S$ be a smooth algebraic curve over $\mathbb{C}$. In this appendix, we denote by $K=\mathbb{C}(S)$ the field of rational functions on $S$, not its algebraic closure. Sometimes, we may withdraw a finite set of points from $S$ but still denote by $S$ the resulting affine curve. We write $S^{a n}$ for the Riemann surface attached to $S(\mathbb{C})$. Finally, we fix a nowhere vanishing vector field $\partial \in H^{0}(S, T S)$ on $S$, which we identify with a derivation of $K$, with constant subfield $\mathbb{C}$.

We start with a commutative algebraic group $G / K$, geometrically connected and with split maximal torus. Shrinking $S$ if necessary, we fix a connected group scheme $\pi: \mathbf{G} \rightarrow S$ extending $G$ over $S$, all of whose fibers have the same toric and unipotent ranks. We denote by $\mathbf{e}$ its 0 -section and by $L \mathbf{G}$ the pull-back $\mathbf{e}^{*}\left(T_{\mathbf{G} / S}\right)$ of the relative tangent bundle of $\mathbf{G}$ over $S$. In other words, $\mathbf{G}$ is an algebraic family $\left\{\mathbf{G}_{t}, t \in S\right\}$ of commutative algebraic groups over $\mathbf{C}$, parametrized by $S$, and $L \mathbf{G}$ is the algebraic family of their tangent spaces $L \mathbf{G}_{t}$ at the origin. At the generic point of $S$, we have the algebraic group $G / K$ with (relative) tangent bundle $T_{G / K} \simeq G \times L G$; this is denoted by $T(G)$ in Section 2 of the text.

Remark A.1. We will also need to consider $\mathbf{G}$ as an analytic family $\mathbf{G}^{\text {an }}$ of complex Lie groups over the Riemann surface $S^{a n}$. We will drop the exponents ${ }^{a n}$ when the context is clear. Moreover, for notational ease, several results below are written at the generic point of $S$, but actually extend to $S$, i.e., can be "bold-faced". We can then "analyticize" them, i.e., add ${ }^{a n}$ both on the base and on the fiber spaces under consideration.

The (total) tangent bundle $T \mathbf{G}$ of $\mathbf{G}$ sits in an exact sequence

$$
0 \rightarrow T_{\mathbf{G} / S} \rightarrow T \mathbf{G} \rightarrow \pi^{*}(T S) \rightarrow 0
$$

of vector bundles over $\mathbf{G}$, and is also a group scheme over $T S$. When $t$ runs through $S$, its fibers $(T \mathbf{G})_{\left(t, \partial_{t}\right)}$ yield a subgroup scheme $T_{\partial} \mathbf{G}$ over $S$, whose generic fiber is called the twisted tangent bundle $T_{\partial} G / K$. A section $\mathbf{y}$ of $\mathbf{G} / S$ provides a section $d \mathbf{y}$ of $T \mathbf{G} / T S$, hence a section $d \mathbf{y}(\partial)$ of $T_{\partial} \mathbf{G} / S$, which in accordance with the text, we denote by $(y, \partial y) \in T_{\partial} G(K)$, or sometimes just $\partial y$, at the generic point of $S$. Viewed over $K, T_{\partial} G$ is a group extension of $G$ by $L G$ (in particular, there is a canonical identification of $L G$ with the fiber above $e$ of $T_{\partial} G$; cf. Section 2.2 of the text and [31, Section 2). The zero section of $T_{\partial} \mathbf{G}$ is $d \mathbf{e}(\partial)$, written $(e, \partial e) \in T_{\partial} G$ at the generic point of $S$.

Viewed over $G, T_{\partial} G$ is a torsor under $T_{G / K}$, and as such, is described by a class in $H^{1}\left(G, T_{G / K}\right)$. Assume now that $\pi$ is proper. We can then consider the 
Kodaira-Spencer map $\kappa: T_{t} S \rightarrow H^{1}\left(\mathbf{G}_{t}, T_{\mathbf{G}_{t} / \mathbb{C}(t)}\right)$ attached to the abelian scheme $\mathbf{G} / S$ at the generic point $t$ of $S$. By definition, its value $\kappa(\partial)$ at $\partial$ is the class of the torsor $T_{\partial} G$. Actually, properness is not required to carry out this construction. In the proper case, it is classical that $\kappa(\partial)$ vanishes if and only if the abelian variety $G / K$ descends to $\mathbb{C}$. See Lemma 3.4 (iii) of the text for the semi-abelian case, and Lemma 3.4 (ii) for counterexamples in the general case.

\section{B. The functors $T_{\partial}, L$ on algebraic groups.}

As explained in Section 2.3 of the text, $T_{\partial}$ and $L$ are functorial: given a morphism of algebraic groups $f: G_{1} \rightarrow G_{2}$, we have the twisted differential $T_{\partial} f: T_{\partial} G_{1} \rightarrow T_{\partial} G_{2}$, with a commutative diagram over $f: G_{1} \rightarrow G_{2}$, and the (vertical) differential at $e_{1}$ of $f$, namely $L f: L G_{1} \rightarrow L G_{2}$. All this can be bold-faced, i.e., comes from group schemes over $S$, where $L$ now stands for $d_{\mathbf{G} / S, \mathbf{e}}$.

We will sometimes apply to the algebraic group $T_{\partial} G / K$ itself (and to its extension over $S$ ) what we are doing on $G$. The following identifications will be crucial.

Lemma B.1. Let $\mathbf{G} / S$ be a group scheme as above. There is a functorial isomorphism between the group schemes $L\left(T_{\partial} \mathbf{G}\right)$ and $T_{\partial}(L \mathbf{G})$. More precisely, given $\mathbf{f}: \mathbf{G}_{1} \rightarrow \mathbf{G}_{2}$, we can identify $T_{\partial}(L \mathbf{f}): T_{\partial}\left(L \mathbf{G}_{1}\right) \rightarrow T_{\partial}\left(L \mathbf{G}_{2}\right)$ with $L\left(T_{\partial} \mathbf{f}\right)$ : $L\left(T_{\partial} \mathbf{G}_{1}\right) \rightarrow L\left(T_{\partial} \mathbf{G}_{2}\right)$.

Proof. This is a straightforward extension over $S$ of Lemma 2.1 of the text, whose proof can be viewed as the study of the functor $T$ itself, i.e., of the total differential $d \mathbf{f}$ on $T \mathbf{G}_{1}$. In what follows, we often write these identifications only at the generic point of $S$. Notice that the formula $L T_{\partial}=T_{\partial} L$ is compatible with the identification of $L G$ with $\left(T_{\partial} G\right)_{e}$, so that the two $L L$ 's which they provide coincide. Also, recall that $L L G$ is canonically isomorphic to $L G$ (more generally, when $V$ is a vectorial group, we always identify $V$ and $L V$, but we sometimes keep to the notation $L V$ to remove ambiguities).

For later use, we point out that

$$
T_{\partial} f\left(y_{1}, \partial y_{1}\right)=\left(f\left(y_{1}\right), \partial\left(f\left(y_{1}\right)\right)\right)
$$

for any $y_{1} \in G_{1}$, and that $T_{\partial} f$ induces $L f$ on the fiber $\left(T_{\partial} G_{1}\right)_{e_{1}}$, identified with $L G_{1}$.

C. Algebraic $D$-groups and $\partial \ell n_{\mathbf{G}}$.

As in Section 2.2 of the text, we now assume that the group extension $T_{\partial} G$ is trivial, in other words, that its class in $H^{1}\left(G, T_{G / K}\right)$ vanishes, and we let $s$ be a homomorphic section of $T_{\partial} G \rightarrow G$, or equivalently, a vector field on $G$ above $\partial$ such that the corresponding derivation on $\mathcal{O}_{G}$ respects the group structure of $G$. We then say that $(G, s)$ is a (commutative) algebraic $D$-group over $K$, and we denote by ADG the corresponding category. The logarithmic derivative of $(G, s)$ (which should be indexed by $s$ ) is then defined by

$$
\partial \ell n_{G}: G \rightarrow\left(T_{\partial} G\right)_{e} \simeq L G: y \mapsto \partial y-s(y) .
$$

Shrinking $S$ if necessary, we can extend $s$ to a section $\mathbf{s}: \mathbf{G} \rightarrow T_{\partial} \mathbf{G}$ over $S$, and we then set

$$
\partial \ln _{\mathbf{G}}: \mathbf{G} \rightarrow\left(T_{\partial} \mathbf{G}\right)_{e} \simeq L \mathbf{G}: \mathbf{y} \mapsto d \mathbf{y}(\partial)-\mathbf{s}(\mathbf{y}) .
$$

When $V$ is a vectorial group over $K$ (i.e., $\mathbf{V} / S$ is a vector bundle), $\partial \ell n_{V}: V \rightarrow V$ and $\partial \ell_{n} \mathbf{V}: \mathbf{V} \rightarrow \mathbf{V}$ are the contractions with $\partial$ of connections in the usual sense. 
We are going to associate to the logarithmic derivative $\partial \ell_{n_{G}}$ two (contracted with $\partial$ ) connections on the vectorial group $L G$ :

- an algebraic one: $\partial_{L G}$ (which is the one of the text itself);

- an analytic one: $\exp _{\mathbf{G}}^{*}\left(\partial \ell n_{\mathbf{G}}\right)=\partial \ell n_{\mathbf{G}} \circ \exp _{\mathbf{G}}$;

this second one is actually defined on $L \mathbf{G}^{a n}$, but see Remark G.6 for a formal approach. We will compare them, in the case $G$ has a unique structure of $A D G$, to:

- the Gauss-Manin connection $\nabla_{L G, \partial}$ (again, algebraic).

More precisely, withdrawing some points of $S$ if necessary, the first one extends over $S$, i.e., is the value at the generic point of $S$ of a connection $\partial_{L \mathbf{G}}$ on the vector bundle $L \mathbf{G}$, and we can look at it analytically, as one on $L \mathbf{G}^{a n}$. Ditto for the third, one of whose characterizations (see Sections $H$ and I) comes from $L \mathbf{G}^{a n}$. In this analytic context, we will prove on the one hand that

$$
\partial_{L \mathbf{G}}=\exp _{\mathbf{G}}^{*}\left(\partial \ell n_{\mathbf{G}}\right),
$$

and on the other hand that

$$
\nabla_{L \mathbf{G}, \partial}=\exp _{\mathbf{G}}^{*}\left(\partial \ell n_{\mathbf{G}}\right)
$$

so that $\partial_{L \mathbf{G}}=\nabla_{L \mathbf{G}, \partial}$. All this is on $\left(L \mathbf{G}^{a n}\right) / S^{a n}$, but these are equalities, not just isomorphisms. So, we will finally deduce that for any almost semi-abelian $D$-group $G$,

$$
\partial_{L G}=\nabla_{L G, \partial} \text { on } L G .
$$

However, the relation with $\exp _{\mathbf{G}}^{*}\left(\partial \ell n_{\mathbf{G}}\right)$ will also be useful for other parts of our paper.

D. The connection $\partial_{L G}$ on $L G$.

As explained in Section 2.3 of the text, this is easy to define in view of Lemma B.1, but we repeat the argument in order to specify which shrinking of $S$ may be necessary. The morphism of algebraic groups $s: G \rightarrow T_{\partial} G$ has a vertical differential $L s: L G \rightarrow L T_{\partial} G$ at the zero section $e$, which, under the identification $L T_{\partial} G=T_{\partial} L G$, can be viewed as a section of $T_{\partial} L G \rightarrow L G$. We have then defined

$$
\partial_{L G}:=\partial \ell n_{(L G, L s)}
$$

as the logarithmic derivative of the ADG structure on $L G$ defined by this section, i.e.,

$$
\partial_{L G}: L G \rightarrow L L G=L G: x \mapsto \partial x-L s(x) .
$$

This is a connection on the vector group $L G / K$.

Now, restricting $S$ if necessary, we may assume that $s$ extends to an $\mathcal{O}_{S}$-section $\mathbf{s}$ of $T_{\partial} \mathbf{G} \rightarrow \mathbf{G}$. Then $L s$ extends over the same base to an $\mathcal{O}_{S}$-homomorphism $L \mathbf{s}=d_{\mathbf{G} / S, \mathbf{e}}(\mathbf{s}): L \mathbf{G} \rightarrow L T_{\partial} \mathbf{G}$. With the identifications of Lemma B.1 in mind, the formula

$$
L \mathbf{G} \ni \mathbf{x} \mapsto \partial_{L \mathbf{G}} \mathbf{x}=d \mathbf{x}(\partial)-L \mathbf{s}(\mathbf{x}) \in L \mathbf{G}
$$

then defines (the contraction with $\partial$ of) a connection on the vector bundle $L \mathbf{G}$, which coincides with $\partial_{L G}$ at the generic point of $S$.

We point out that the description (given at the end of Section 2.3) of $\partial_{L G}$ as the differential $L \partial \ell n_{G}$ of $\partial \ell n_{G}$ at the identity, in the sense of Kolchin's differential algebraic geometry, could also be carried out in the present setting. 
Remark D.1. Let $V$ be a vectorial subgroup of $G$. If $V$ is an ADG subgroup of $(G, s)$, i.e., if $s(V) \subset T_{\partial} V \subset T_{\partial} G$, i.e., if $s$ induces a section $s_{V}$ of $T_{\partial} V \rightarrow V$, the differential $L s_{V}: L V \simeq V \rightarrow L T_{\partial} V \simeq T_{\partial} V$ of $s_{V}$ can be identified with $s_{V}$, and we get

$$
\left.\left(\partial_{L G}\right)\right|_{L L V=L V}=\left.\left(\partial \ell n_{G}\right)\right|_{L V=V} .
$$

We will show in Corollary G.4 below that this relation still holds true when the vectorial subgroup $V$ is not an ADG subgroup of $G$.

\section{E. The exponential map on $\mathbf{G}^{a n}$.}

For each $t \in S$, we can consider the exponential map $\exp _{\mathbf{G}_{t}}: L \mathbf{G}_{t}(\mathbb{C}) \rightarrow \mathbf{G}_{t}(\mathbb{C})$ of the connected Lie group $\mathbf{G}_{t}^{a n}$ attached to $\mathbf{G}_{t}(\mathbb{C})$. Its kernel $\mathcal{P}_{t}$ is the $\mathbb{Z}$-module of periods of $\mathbf{G}_{t}^{a n}$. These patch into an exact sequence of analytic sheaves of abelian groups over $S^{a n}$ :

$$
0 \rightarrow \mathcal{P} \rightarrow L \mathbf{G}^{a n} \rightarrow \mathbf{G}^{a n} \rightarrow 0,
$$

whose third arrow $\exp _{\mathbf{G}^{a n}}$ induces $\exp _{\mathbf{G}_{t}}$ above each $t$. Its kernel $\mathcal{P}$ will be described in Section H. Following Remark A.1, we will drop the exponents ${ }^{a n}$ when the exponential morphism is concerned. Typically, $\exp _{\mathbf{G}}$ can only mean $\exp _{\mathbf{G}^{a n}}$, with its source the analytic vector bundle $L\left(\mathbf{G}^{a n}\right)=(L \mathbf{G})^{a n}$ over $S^{a n}$.

Let us now collect some properties of this $S^{a n}$-morphism $\exp _{\mathbf{G}}: L \mathbf{G}^{a n} \rightarrow \mathbf{G}^{a n}$. Writing the group law on $G$ additively, it is characterized by the joint conditions that:

(i) $\forall U \subset S^{a n}, \forall \mathbf{x}_{1}, \mathbf{x}_{2} \in L \mathbf{G}^{a n}(U), \exp _{\mathbf{G}}\left(\mathbf{x}_{1}+\mathbf{x}_{2}\right)=\exp _{\mathbf{G}}\left(\mathbf{x}_{1}\right)+\exp _{\mathbf{G}}\left(\mathbf{x}_{2}\right)$;

(ii) $L \exp _{\mathbf{G}}=i d_{L \mathbf{G}}$ (under the usual identification $L L \mathbf{G}=L \mathbf{G}$ ).

Here, $\operatorname{Lxp}_{\mathbf{G}}=d_{\mathbf{G} / S, \mathbf{e}} \exp _{\mathbf{G}}$ means the vertical differential of $\exp _{\mathbf{G}}$ along the zero section $\mathbf{e}$, which is still meaningful in the analytic setting.

So, we must repeat Sections A and B in their entirety in the context of analytic sheaves of abelian groups over $S^{a n}$. More precisely, the situation is as follows: we have two (algebraic) group schemes $\mathbf{G}_{1}, \mathbf{G}_{2}$ and an analytic morphism $\phi: \mathbf{G}_{1}^{\text {an }} \rightarrow$ $\mathbf{G}_{2}^{a n}$ over $S^{a n}$. We can then define $T_{\partial} \phi: T_{\partial} \mathbf{G}_{1}^{a n} \rightarrow T_{\partial} \mathbf{G}_{2}^{a n}, L \phi: L \mathbf{G}_{1}^{a n} \rightarrow L \mathbf{G}_{2}^{a n}$, we can again identify the $S^{a n}$-sheaves $T_{\partial} L \mathbf{G}_{i}^{a n}$ and $L T_{\partial} \mathbf{G}_{i}^{a n}$, and get $L T_{\partial} \phi=T_{\partial} L \phi$. A typical example will be given by

$$
\mathbf{G}_{1}=L \mathbf{G}, \mathbf{G}_{2}=\mathbf{G}, \phi=\exp _{\mathbf{G}} .
$$

From (i) and (ii), we immediately deduce the well-known property:

(iii) $\forall \phi: \mathbf{G}_{1}^{a n} \rightarrow \mathbf{G}_{2}^{a n}$, we have $\phi \circ \exp _{\mathbf{G}_{1}}=\exp _{\mathbf{G}_{2}} \circ L \phi$, and the important fact that

(iv) if $\mathbf{V}$ is a vectorial group scheme (= vector bundle) over $S$, then $\exp _{\mathbf{V}}=i d_{L \mathbf{V}}$ in the usual identification $L \mathbf{V}=\mathbf{V}$.

If $G_{1}$ is a subgroup of $G_{2}$, property (iii) shows that $\exp _{\mathbf{G}_{1}}$ is the restriction to $L \mathbf{G}_{1}$ of $\exp _{\mathbf{G}_{2}}$. Consider in particular the exponential map $\exp _{T_{\partial} \mathbf{G}}: L T_{\partial} \mathbf{G} \rightarrow T_{\partial} \mathbf{G}$ of the group scheme $T_{\partial} \mathbf{G}$. Its restriction to the Lie algebra $L L \mathbf{G}=L \mathbf{G}$ of the vectorial subgroup $\mathbf{G}_{1}=L \mathbf{G}$ of $\mathbf{G}_{2}=T_{\partial} \mathbf{G}$ is the exponential map $\exp _{L \mathbf{G}}$ of $L \mathbf{G}$. By property (iv), we therefore have (the trivial, but crucial!):

Lemma E.1. For any algebraic group $G$,

(v) $\left.\left(\exp _{T_{\partial} \mathbf{G}}\right)\right|_{L L \mathbf{G}=L \mathbf{G} \subset L T_{\partial} \mathbf{G}}=\exp _{L \mathbf{G}}=i d_{L \mathbf{G}}$.

This property should not be confused with (ii). (Recall that we are indexing the exponential maps by the groups, not by their Lie algebras.) 


\section{F. The connection $\exp _{\mathbf{G}}^{*}\left(\partial \ell_{n_{\mathbf{G}}}\right)$ on $L \mathbf{G}^{a n}$.}

Let us make a preliminary comment on pull-backs of connections in the classical case. Let $V_{1}, V_{2}$ be two vector spaces over $K$, let $\nabla_{2}: V_{2} \rightarrow V_{2} \otimes \Omega_{K / \mathbb{C}}^{1}$ be a connection on $V_{2}$, and let $f: V_{1} \rightarrow V_{2}$ be a $K$-linear map. In general we cannot define the pull-back $\nabla_{1}:=f^{*}\left(\nabla_{2}\right)$ of $\nabla_{2}$ under $f$, but we can if $f$ is an isomorphism. Indeed, $\nabla_{1}:=(f \otimes 1)^{-1} \circ \nabla_{2} \circ f$ is a connection on $V_{1}$. Notice that it is the unique connection such that $f:\left(V_{1}, \nabla_{1}\right) \rightarrow\left(V_{2}, \nabla_{2}\right)$ is a horizontal morphism.

Now let $f: G_{1} \rightarrow G_{2}$ be a morphism of commutative algebraic groups over $K$, and let $s_{2}: G_{2} \rightarrow T_{\partial} G_{2}$ be an ADG structure on $G_{2}$. In general, we cannot define the pull-back of $s_{2}$ or of $\partial \ell n_{G_{2}}$ under $f$, but we can if $f$ is an isogeny (i.e., a finite covering). Indeed, for any $y_{1} \in G_{1}$ with $y_{2}=f\left(y_{1}\right), T_{\partial} f$ then induces an isomorphism on the fibers $\left(T_{\partial} G_{1}\right)_{y_{1}} \rightarrow\left(T_{\partial} G_{2}\right)_{y_{2}}$, and we may set $s_{1}\left(y_{1}\right)=\left(\left(T_{\partial} f\right)_{y_{1}}\right)^{-1}\left(s_{2}\left(y_{2}\right)\right): G_{1} \rightarrow T_{\partial} G_{1}$, thereby defining the unique logarithmic derivative $\partial \ell n_{G_{1}}:=f^{*}\left(\partial \ell n_{G_{2}}\right): G_{1} \rightarrow L G_{1}$ on $G_{1}$ such that $f$ is horizontal, in the sense that

$$
L f \circ \partial \ell n_{G_{1}}=\partial \ell n_{G_{2}} \circ f .
$$

Indeed, $\left(T_{\partial} f\right)\left(y_{1}, \partial y_{1}\right)=\left(f\left(y_{1}\right), \partial\left(f\left(y_{1}\right)\right)\right)$, and $L$ is the restriction of $T_{\partial}$ above the zero section so that $(L f)\left(\partial y_{1}-s_{1}\left(y_{1}\right)\right)=\left(T_{\partial} f\right)\left(\left(y_{1}, \partial y_{1}\right)-\left(y_{1}, s_{1}\left(y_{1}\right)\right)\right)=$ $\left(T_{\partial} f\right)\left(y_{1}, \partial y_{1}\right)-\left(T_{\partial} f\right)\left(y_{1}, s_{1}\left(y_{1}\right)\right)=\left(y_{2}, \partial y_{2}\right)-\left(y_{2}, s_{2}\left(y_{2}\right)\right)=\partial\left(y_{2}\right)-s_{2}\left(y_{2}\right) \in L G_{2}$.

Let us note for the record that the last paragraph also shows that if $G_{1}, G_{2}$ are algebraic $D$-groups and $f: G_{1} \rightarrow G_{2}$ is a $D$-homomorphism (homomorphism of algebraic $D$-groups), then

$$
L f \circ \partial \ell n_{G_{1}}=\partial \ell n_{G_{2}} \circ f .
$$

The construction above extends word for word over $S$, and it can therefore be bold-faced and analyticized. Now, more generally, we can consider an analytic morphim $\phi: \mathbf{G}_{1}^{a n} \rightarrow \mathbf{G}_{2}^{a n}$. As soon as $\phi$ is a covering (not necessarily finite, but with discrete kernel), the same construction applies, and parallel with $s_{1}$, we obtain an analytic section $\sigma_{1}: \mathbf{G}_{1}^{a n} \rightarrow T_{\partial} \mathbf{G}_{1}^{a n}$, hence an analytic logarithmic derivative

$$
\partial \ell n_{\mathbf{G}_{1}^{a n}}:=\phi^{*}\left(\partial \ell n_{\mathbf{G}_{2}}\right): \mathbf{G}_{1}^{a n} \rightarrow L \mathbf{G}_{1}^{a n}
$$

on $\mathbf{G}_{1}^{a n}$ such that $L \phi \circ \partial \ell n_{\mathbf{G}_{1}^{a n}}=\partial \ln _{\mathbf{G}_{2}^{a n}} \circ \phi$.

Since $\exp _{\mathbf{G}}$ is an analytic covering, we may apply the latter construction to the situation $\mathbf{G}_{1}=L \mathbf{G}, \mathbf{G}_{2}=\mathbf{G}, \phi=\exp _{\mathbf{G}}$. We thereby obtain (the contraction with $\partial$ of) an analytic connection on $L \mathbf{G}^{a n}$ :

$$
\exp _{\mathbf{G}}^{*}\left(\partial \ell n_{\mathbf{G}}\right): L \mathbf{G}^{a n} \rightarrow L \mathbf{G}^{a n},
$$

under the usual identification of the vector bundles $L L \mathbf{G}=L \mathbf{G}$ over $S$.

The horizontality of $\phi$ (worked out in the algebraic setting of $f$ in the previous section), applied to the present case $\phi=\exp _{\mathbf{G}}$, gives

$$
L \exp _{G} \circ \exp _{\mathbf{G}}^{*}\left(\partial \ell n_{\mathbf{G}}\right)=\partial \ell n_{\mathbf{G}} \circ \exp _{\mathbf{G}},
$$

and since $\operatorname{Lexp}_{\mathbf{G}}=i d_{L \mathbf{G}}$ by property (ii), we eventually have

Lemma F.1. $\exp _{\mathbf{G}}^{*}\left(\partial \ell n_{\mathbf{G}}\right)=\partial \ell n_{\mathbf{G}} \circ \exp _{\mathbf{G}}$. 
G. $\exp _{\mathbf{G}}^{*}\left(\partial \ell n_{\mathbf{G}}\right)=\partial_{L \mathbf{G}}$.

Let $G$ be an arbitrary commutative algebraic $D$-group. So, a section $s: G \rightarrow T_{\partial} G$ is fixed and extended to $s: \mathbf{G} \rightarrow T_{\partial} \mathbf{G}$ as usual. Given a local section $\mathbf{x} \in L \mathbf{G}^{a n}(U)$, we proceed to compute $\exp _{\mathbf{G}}^{*}\left(\partial \ln _{\mathbf{G}}\right)(\mathbf{x})=\partial \ell_{n_{\mathbf{G}}} \circ \exp _{\mathbf{G}}(\mathbf{x})$ and will show:

Proposition G.1. Let $G / K$ be an algebraic D-group. Then, $\exp _{\mathbf{G}}^{*}\left(\partial \ell n_{\mathbf{G}}\right)=\partial_{L \mathbf{G}}$.

However, we will not bold-face everything in this section (not speaking of the already dropped exponents ${ }^{a n}$ ), in the hope that the context is clear.

It is at this point that we need the exponential map of the algebraic group $T_{\partial} G$ itself (more properly, the exponential morphism of the analytic family $T_{\partial} \mathbf{G}^{a n}$ ). So, we have $\exp _{T_{\partial} \mathbf{G}}: L T_{\partial} \mathbf{G}^{a n} \rightarrow T_{\partial} \mathbf{G}^{a n}$, abbreviated as $\exp _{T_{\partial} G}$. By the analytic version of Section B, we may identify its source with $T_{\partial} L \mathbf{G}^{a n}$. Our proof of Proposition G.1 will in particular make use of Lemmas E.1 and F.1.

By definition,

$$
\partial_{L G}(x)=\partial x-L s(x)
$$

while

$$
\partial \ell n_{G}\left(\exp _{G}(x)\right)=\partial\left(\exp _{G}(x)\right)-s\left(\exp _{G}(x)\right) .
$$

By property (iii), $s\left(\exp _{G}(x)\right)=\exp _{T_{\partial} G}(L s(x)) \in T_{\partial} G$, and we claim (see Lemma G.3 below) that $\partial\left(\exp _{G}(x)\right)=\exp _{T_{\partial} G}(\partial x) \in T_{\partial} G$. So,

$$
\partial \ln _{G}\left(\exp _{G}(x)\right)=\exp _{T_{\partial} G}(\partial x)-\exp _{T_{\partial} G}(L s(x))=\exp _{T_{\partial} G}(\partial x-L s(x)) .
$$

But $\partial x-L s(x)=(x, \partial x)-(x, L s(x))$ is a point of $T_{\partial} L G=L T_{\partial} G$ lying in the Lie algebra $L L G$ of the vectorial subgroup $L G$ of $T_{\partial} G$. By property (v) in Lemma E.1, we therefore have

$$
\exp _{T_{\partial} G}(\partial x-L s(x))=i d_{L G}(\partial x-L s(x))=\partial x-L s(x)=\partial_{L G}(x),
$$

and the proposition is established.

Remark G.2. This computation, where we lift questions on $G$ to $T_{\partial} G$ and then use the vectorial properties of its subgroup $L G$, is reminiscent of methods from universal vectorial extensions. But notice that our algebraic $D$-group $G$ is already (essentially) a universal extension.

It remains to show that

Lemma G.3. For any algebraic group $G$ and any $x$ in $L G$, we have $\partial\left(\exp _{G}(x)\right)=$ $\exp _{T_{\partial} G}(\partial x) \in T_{\partial} G$.

Proof. We already know (by the analytic version of the last relation in Section B) that $\partial\left(\exp _{G}(x)\right)=\left(T_{\partial} \exp _{G}\right)(\partial x)$. We must now prove that

$$
T_{\partial} \exp _{G}=\exp _{T_{\partial} G}
$$

Since $T_{\partial} \exp _{G}$ is a group morphism, we are reduced, in view of the properties (i) and (ii) characterizing the exponential morphism $\exp _{T_{\partial} G}$ of $T_{\partial} G$, to check that

$$
L T_{\partial} \exp _{G}=i d_{L T_{\partial} G} \text {. }
$$

By the analytic version of Lemma B.1, $L T_{\partial} \exp _{G}=T_{\partial} L \exp _{G}$, and $L \exp _{G}=i d_{L G}$ by property (ii) for $\exp _{G}$. So, the LHS is $T_{\partial} i d_{L G}$. Now, the RHS is $i d_{L T_{\partial} G}=i d_{T_{\partial} L G}$, and the requested identity

$$
T_{\partial} i d_{L G}=i d_{T_{\partial} L G}
$$

actually holds true for any algebraic group, not necessarily of the type $L G$. 
Corollary G.4. Let $V / K$ be an arbitrary vectorial subgroup of $G$, identified with its Lie algebra $L V \subset L G$ and with $L L V \subset L L G$. Then

$$
\left.\left(\partial_{L G}\right)\right|_{V}=\left.\left(\partial \ell n_{G}\right)\right|_{V} \text {. }
$$

In particular, $V$ is an $A D G$ subgroup of $G$ if and only if it is a $\partial_{L G \text {-submodule of }}$ $L G$.

Proof. In view of property (iv) of the exponential map $\exp _{V}=\left.\exp _{G}\right|_{L V}$, we deduce from Proposition G.1 that indeed,

$$
\partial_{L G}(v)=\partial \ell n_{G}\left(\exp _{G}(v)\right)=\partial \ell n_{G}(v)
$$

for any $v \in V$. Notice that contrary to Remark D.1, this formula was not immediately clear. In our paper, it will replace the role of Remark 1.4 of [9], p. 64. It also clarifies the role of hypothesis $(\mathbf{H})$ at the end of Section I.

The last sentence of Corollary G.4 can be stated in greater generality, as follows.

Corollary G.5. Let $H / K$ be a connected algebraic subgroup of $G$ with Lie algebra $L H$. Then, $H$ is an ADG subgroup of $G$ if and only if $L H$ is a $\partial_{L G \text {-submodule of }}$ $L G$.

Proof. Left to right, which is a special case of Lemma 2.5 of the text, it is clear from the definition of $\partial_{L G}$ with sections. For right to left, notice that $\exp _{H}=\left.\exp _{G}\right|_{L H}$, so that for any local section $\mathbf{y}:=\exp _{\mathbf{G}}(\mathbf{x}), \mathbf{x} \in L \mathbf{H}$ of $\mathbf{H}$ over a small disk $U$ in $S, \partial \ell n_{\mathbf{G}}(\mathbf{y})=\partial_{L \mathbf{G}}(\mathbf{x}) \in L \mathbf{H}(U)$. Therefore, for any $y \in H, \partial \ell n_{G}(y)$ lies in $L H \simeq\left(T_{\partial} H\right)_{e}$, and $s(y)=\partial y-\partial \ell n_{G}(y)$ does lie in $T_{\partial} H$.

Remark G.6. In both Sections D and E, we used only the properties of the exponential morphism of the formal group scheme $\hat{\mathbf{G}}=$ formal completion of $\mathbf{G}$ along the zero section e. This formal exponential is again entirely characterized by properties (i) and (ii) of Section D. Consequently, the whole development of these sections goes through, with $\mathbf{G}^{a n}$ replaced by $\hat{\mathbf{G}}$. The complex-analytical properties of $\exp _{\mathbf{G}}$, reflected in the study of the full exact sequence $(\dagger)$, are needed only in the next sections.

H. Gauss-Manin: $\nabla_{L \mathbf{G}, \partial}=\exp _{\mathbf{G}}^{*}\left(\partial \ell n_{\mathbf{G}}\right)$.

Given a connected commutative algebraic group $G / K$, we extended it to a group scheme $\mathbf{G} / S$ and considered in Section E the exponential sequence

$$
0 \rightarrow \mathcal{P} \rightarrow L \mathbf{G}^{a n} \rightarrow \mathbf{G}^{a n} \rightarrow 0 .
$$

Recall now from Section A that we are assuming that the fibers $\mathbf{G}_{t}, t \in S(\mathbb{C})$, have constant toric and unipotent ranks, which amounts to their having constant topological type. This can always be achieved by removing a finite set of points from $S$. Under this assumption, the kernel $\mathcal{P}$ of $(\dagger)$ is a local system over $S^{a n}$, dual to the local system $R^{1} \pi_{*}(\mathbb{Z})$ formed by the Betti cohomology groups of the fibers $\mathbf{G}_{t}$. For any open $U \subset S^{a n}$, the sections in $\mathcal{P}(U) \subset L \mathbf{G}^{a n}(U)$ are killed by the exponential morphism $\exp _{\mathbf{G}}$. In particular, as soon as $G$ is an algebraic $D$ group, Lemma F.1 shows that they are horizontal sections of the analytic connection $\exp _{\mathbf{G}}^{*}\left(\partial \ell n_{\mathbf{G}}\right)=\partial \ell n_{\mathbf{G}} \circ \exp _{\mathbf{G}}$. In view of Proposition G.1, the local sections of $\mathcal{P}$ are therefore horizontal for the connection $\partial_{L \mathbf{G}}$ :

$$
\forall U \subset S^{a n}, \forall \lambda_{U} \in \mathcal{P}(U), \partial_{L \mathbf{G}}\left(\lambda_{U}\right)=0 .
$$


Now, assume that $L \mathbf{G}^{a n}$ is locally generated over $\mathcal{O}_{S^{a n}}$ by $\mathcal{P}$. There then exists at most one connection on $L \mathbf{G}^{a n}$ killing $\mathcal{P}$. So, any connection on $L \mathbf{G}^{\text {an }}$ killing $\mathcal{P}$ will coincide with $\exp _{\mathbf{G}}^{*}\left(\partial \ell n_{\mathbf{G}}\right)=\partial_{L \mathbf{G}}$. This is the principle, borrowed from [24, on which the whole present section is based.

We are now going to define the Gauss-Manin connection $\nabla_{L G}$ on $L G$ when $G$ is an almost semi-abelian $D$-group. The definition is that of [8] when $G$ is the universal vectorial extension of a semi-abelian variety, and the general case follows by taking a quotient. As in the text, we will use the following notation. We write $B$ for the maximal semi-abelian quotient of $G$, and $\tilde{B}$ for the universal vectorial extension of $B$. Therefore, $B$ is an extension of an abelian variety $A / K$ by a torus $T$, which we have assumed to be split over $K$. Recall from Lemma 3.4 (ii) that $\tilde{B}$ carries a unique structure of an algebraic $D$-group. By definition of an almost semi-abelian $D$-group (cf. Section 3.1 of the text), there exists a canonical vectorial subgroup $V$ of $\tilde{B}$, which is an algebraic $D$-subgroup of $\tilde{B}$, and such that $G=\tilde{B} / V$. We endow $G$ with the quotient ADG structure, which is actually the unique ADG structure one can put on $G$; see again Lemma 3.4. So, $\partial \ell n_{\tilde{B}}$ and $\partial \ell n_{G}$ are well defined.

In these conditions, the topological hypothesis made on the fibers of the group scheme $\mathbf{G} / S$ implies that $B / K$ can be continued to an extension $\mathbf{B} / S$ of an abelian scheme $\mathbf{A} / S$ by the constant torus $\mathbf{T}=T \times S$. In other words, the one-motive $M=[0 \rightarrow \mathbf{B}]$ is smooth over $S$ in the sense of Deligne [15], III, 10.1.10. Then, $[0 \rightarrow \tilde{\mathbf{B}}]$ is the universal vectorial extension of $M$, and the vector bundle $L \tilde{\mathbf{B}}$ is its de Rham realization $T_{d R}(M)$; see [15, loc. cit., also 3]. Finally, we denote by $\tilde{\mathcal{P}} \subset L \tilde{\mathbf{B}}$ and $\overline{\mathcal{P}} \subset L \mathbf{B}$ the kernels of the exponential exact sequences of the sheaves $\tilde{\mathbf{B}}^{a n}$ and $\mathbf{B}^{a n}$ over $S^{a n}$. In particular, $\overline{\mathcal{P}}$ is the Betti realization $T_{\mathbb{Z}}(M)$ of the one-motive $M$.

Since the exponential maps have no kernel on vectorial groups, the local sys-

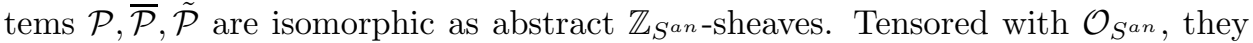
all define the same vector bundle, say $\mathcal{P} \otimes \mathcal{O}_{S^{a n}}$, which, as said above, carries a unique connection $\nabla=i d_{\mathcal{P}} \otimes d_{S^{a n} / \mathbb{C}}$ (equivalently $\bar{\nabla}, \tilde{\nabla}$ ) relative to which they are horizontal. Now, by [8], Facts 2.2.2.1 and 2.2.2.2, we have:

Fact H.1. The natural map

$$
\tilde{\mathcal{P}} \otimes \mathcal{O}_{S^{a n}} \rightarrow L \tilde{\mathbf{B}}^{a n}
$$

is an isomorphism of vector bundles over $S^{a n}$, and there exists an algebraic connection $\nabla_{L \tilde{\mathbf{B}}}$ on the $\mathcal{O}_{S}$-module $L \tilde{\mathbf{B}}=T_{d R}(M)$ such that $\tilde{\nabla}$ and $\nabla_{L \tilde{\mathbf{B}}^{\text {an }}}$ coincide under this isomorphism (i.e., such that $\nabla_{L \tilde{\mathbf{B}}}$ kills the local sections of $\tilde{\mathcal{P}}$ ). We define the Gauss-Manin connection $\nabla_{L \tilde{B}}$ on $L \tilde{B}$ as the connection induced by $\nabla_{L \tilde{\mathbf{B}}}$ at the generic point of $S$.

This fact reflects the horizontality of the canonical pairing "integration of forms against cycles" between $T_{\mathbb{Z}}(M)=\overline{\mathcal{P}} \otimes \mathcal{O}_{S^{a n}}$, endowed with $\bar{\nabla}$, and the vector bundle $H_{d R}^{1}(\mathbf{B} / S) \simeq T_{d R}(M)^{*}$ formed by the de Rham cohomology groups of the fibers of the semi-abelian scheme $\mathbf{B} / S$, endowed with the algebraic Gauss-Manin connection, as originally defined in [18. For a slightly different presentation, see [1] and [33].

Fact H.2. When $B=A$ is an abelian variety, $\nabla_{L \tilde{\mathbf{A}}}$ reduces to the dual of the classical Gauss-Manin connection on the de Rham cohomology group $H_{d R}^{1}(\mathbf{A} / S)$, 
as described in [15, II, 14]. Notice that part (ii) of the corollary below, restricted to the study of $\nabla_{L \tilde{\mathbf{A}}}$, is the only property of Gauss-Manin connections needed in the text (see Section 4.2, (I), (II)).

NB: in this proper case $B=A$, the connection $\nabla_{p}$ described by Buium in [9], Chapter 3.1, Remark 1.4, p. 64, coincides with the above $\nabla_{L \tilde{A}, \partial}$. For an extension to the general case, see [9], Chapter 3.2, Theorem 2.2 (3).

Fact H.3. In the general case, $\nabla_{L \tilde{\mathbf{B}}}$ is an extension, in the category of $D$-modules over $S$, of $\nabla_{L \tilde{\mathbf{A}}}$ by $\nabla_{L \mathbf{T}}$ (the latter one is a direct sum of copies of the trivial $D$-module $\left.\left(\mathcal{O}_{S}, d\right)\right)$. In other words, $L \mathbf{T}$ is stable under $\nabla_{L \tilde{\mathbf{B}}}$.

This merely means that the Gauss-Manin connection respects the weight filtration of the smooth one-motive $M$. This standard fact from [8], 2.2.2.1, reflected on each fiber $\tilde{\mathbf{B}}_{t}$ by [15, III.10.1.8, can in fact also be deduced from Corollary 3.7 of the text, combined with (the easy side of) Corollary G.5 above.

By the definition in Fact H.1, $\nabla_{L \tilde{\mathbf{B}}}$ kills the local system $\tilde{\mathcal{P}} \subset L \tilde{\mathbf{B}}^{\text {an }}$. The principle recalled earlier therefore implies

$$
\nabla_{L \tilde{\mathbf{B}}, \partial}=\exp _{\tilde{\mathbf{B}}}^{*}\left(\partial \ell n_{\tilde{\mathbf{B}}}\right) .
$$

Finally, the vectorial subgroup $V$ of $\tilde{B}$ such that $G=\tilde{B} / V$ is by hypothesis a $D$ subgroup of $G$. By Remark D.1 (or the easy side of Corollary G.5), $V=L V \subset L \tilde{B}$ is stable under the connection $\partial_{L \tilde{B}}$, i.e., under $\exp _{\tilde{B}}^{*}\left(\partial \ell n_{\tilde{B}}\right)$ (see Section G), i.e., under $\nabla_{L \tilde{B}}$ (by what has just been proved). We may therefore define the algebraic Gauss-Manin connection $\nabla_{L G}$ on $L G=L \tilde{B} / V$ as the quotient connection induced by $\nabla_{L \tilde{B}}$ (and everything can be bold-faced). Since $\nabla_{L \mathbf{G}}$ kills the image $\mathcal{P}$ of $\tilde{\mathcal{P}}$ in $L \mathbf{G}^{a n}$, and since $\mathcal{P}$ still generates $L \mathbf{G}^{a n}$ locally, the principle again gives:

Proposition H.4. Let $G / K$ be an almost semi-abelian D-group. Then exp $\mathbf{G}_{\mathbf{G}}^{*}\left(\partial \ell_{n_{\mathbf{G}}}\right)$ $=\nabla_{L \mathbf{G}, \partial}$ on $L \mathbf{G}^{a n}$.

At long last:

Corollary H.5. Let $G / K$ be an almost semi-abelian D-group, let $B$ be its maximal semi-abelian quotient, let $T$ be its toric part, and let $A_{0}$ be the $K / \mathbb{C}$-trace of its maximal abelian quotient $A$. Then:

(i) $\partial_{L G}$ coincides with the Gauss-Manin connection $\nabla_{L G, \partial}$.

(ii) Assume that $B=A \times T$. Then:

(I) the connection $\partial_{L G}$ on $L G$ is semi-simple;

(II) its $K$-rational horizontal vectors lie in the image $L G_{0}(\mathbb{C})$ of $L \tilde{A}_{0}(\mathbb{C}) \times L T(\mathbb{C})$ in $L G$.

(iii) In all cases, $T$ is an $A D G$ subgroup of $G$.

Proof. Combining Propositions G.1 and H.4, we get (i). Assertion (ii), which only requires Fact H.2, then follows from the semi-simplicity theorem of [15], II, Théorème 4.2.6, and from the theorem of the fixed part of loc. cit., Corollaire 4.1.2. As for (iii), it of course directly follows from Corollary 3.7 of the text, as pointed out in the proof of Lemma 3.13 (i). But notice that it also follows from Fact H.3, together with the less obvious side of Corollary G.5.

Remark H.6. In the general case where $B$ is a non-isotrivial extension, the first part of assertion (ii) of this corollary does not hold, i.e., $\nabla_{L G}$ is not semi-simple: 
this can be deduced from the version of Manin's theorem of the kernel studied in Section J, applied to the dual of $A$. The theorem of the fixed part of 33] provides an analogue of the second part of (ii), as illustrated by Claim III of Section 5.3 of the text.

\section{Almost semi-abelian D-groups: An analytic approach.}

Here we give a different approach to Section H, which still relies on Fact H.1 to deal with universal extensions (and marginally on Fact H.3), but which may be nicer as it defines $\nabla_{L G}$ directly on $L G$, with no prior analysis of $G$. Let $G / K$ be any commutative algebraic group, not necessarily an ADG. By Chevalley's theorem, it is a vectorial extension of its maximal semi-abelian quotient $B$. So, $G$ is a push-out of the universal vectorial extension $\tilde{B}$ of $B$, but not necessarily a quotient of $\tilde{B}$.

Consider the natural map

$$
j: \mathcal{P} \otimes \mathcal{O}_{S^{a n}} \rightarrow L \mathbf{G}^{a n} .
$$

We know that $\mathcal{P} \otimes \mathcal{O}_{S^{a n}}$ carries a unique connection $\nabla$ killing the local system $\mathcal{P}$. By Fact H.1 of the previous section, combined with the isomorphism $\mathcal{P} \simeq \tilde{\mathcal{P}}$, we know that $\nabla \simeq \tilde{\nabla}$ "is" the algebraic Gauss-Manin connection $\nabla_{L \tilde{\mathbf{B}}}$ on $L \tilde{\mathbf{B}}$ (which we again denote by $\nabla$ below). The discussion now goes as follows.

- Assume that $j$ is an isomorphism, i.e., both that $\underline{\mathbb{Z}}_{S}$-linearly independent periods are $\mathcal{O}_{S}$-linearly independent and that $L \mathbf{G}^{a n}$ is locally generated by $\mathcal{P}$. Then $G=\tilde{B}$, and we just set $\nabla_{L G}:=\nabla$. Notice, as in Malgrange's lecture notes 24, that this hypothesis holds if and only if the fibers $\mathbf{G}_{t}$ of $\mathbf{G} / S$ are all analytically isomorphic to $\mathbb{C}^{n} / \mathbb{Z}^{n} \simeq(\mathbb{C})^{* n}$, i.e., that analytically, they are all isomorphic to a torus $\left(\mathbb{G}_{m}\right)^{n}$. Of course, they will not be so algebraically if $B$ has a non-trivial abelian part (as in Serre's classical counterexample).

- Now, merely assume that $j$ is surjective, i.e., that $L \mathbf{G}^{a n}$ is locally generated

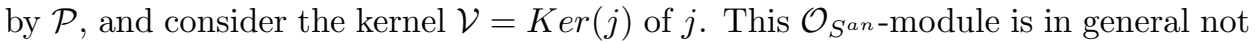
stable under $\nabla$. For instance, if $G=A$ is proper, $\mathcal{V}$ is the first step of the Hodge filtration (cf. [15], II. 4.4.2 and also III. 10.1.3.1), and $\nabla(\mathcal{V}) \subset \mathcal{V}$ if and only if $A$ is constant. But assume further that $\mathcal{V}$ is a sub- $\nabla$-module, i.e., that

- $\quad(\mathbf{H}) \quad \operatorname{Im}(j)=L \mathbf{G}^{\text {an }}$ and $\nabla(\operatorname{Ker}(j)) \subset \operatorname{Ker}(j)$.

Then, we can define $\nabla_{L \mathbf{G}^{a n}}$ as the quotient connection which $\nabla$ induces on $L \mathbf{G}^{a n}=\left(\mathcal{P} \otimes \mathcal{O}_{S^{a n}}\right) / \mathcal{V}=L \tilde{\mathbf{B}}^{a n} / \mathcal{V}$. We do not yet know whether $\nabla_{L \mathbf{G}^{a n}}$ is algebraic.

Let us now show that it is, thereby allowing us to write it $\nabla_{L G}$ at the generic point of $S$ (and finally, to call it the Gauss-Manin connection on $L G$ ). More precisely:

Proposition I.1. (i) under $(\mathbf{H})$, the connection $\nabla_{L \mathbf{G}^{\text {an }}}$ comes from an algebraic connection $\nabla_{L \mathbf{G}}$ on $L \mathbf{G}$;

(ii) $(\mathbf{H})$ holds only if (and if) $G$ is an almost semi-abelian D-group.

Proof. i) From Fact H.3 and the well-known fuchsianity of the Gauss-Manin connection in the proper case as in Fact H.2, we deduce that $\nabla$ too is a fuchsian

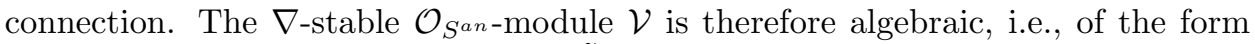
$\mathbf{V}^{a n}$, for some $\mathcal{O}_{S}$-submodule $\mathbf{V}$ of $L \tilde{\mathbf{B}}$. So, the quotient connection is indeed algebraic. Let us temporarily denote by $V^{\prime}$ the $K$-vector subspace that $\mathbf{V}$ defines at the generic point of $S$, so that $L G=L \tilde{B} / V^{\prime}$.

ii) First, the surjectivity of $j$ implies that $G$ admits no $\mathbb{G}_{a}$ factor, i.e., is a quotient $\tilde{B} / V$ (rather than just a push-out) of $\tilde{B}$ by some vectorial subgroup $V / K$ 
of $\tilde{B}$. From the relation $L G=L \tilde{B} / V^{\prime}$, we deduce that $L V=V^{\prime}$. In particular, $L V$ is stable under $\nabla=\nabla_{L \tilde{B}}$, which is equal to $\partial_{L \tilde{B}}$ by the main principle of the previous section. Now, the less immediate part of Corollary G.5 (in fact, here, of Corollary G.4) implies that $V$ is an algebraic $D$-subgroup of $\tilde{B}$. Therefore, $G$ is indeed an almost semi-abelian $D$-group.

The converse implication is easier; see the previous section. Our point here is that the algebraic notion of an almost semi-abelian D-group is entirely described by the analytic hypothesis $(\mathbf{H})$, whose first assumption can be viewed as a transcendental analogue of Lemma 3.1 (iii) of the text.

\section{Conclusion.}

Given an algebraic $D$-group $G / K$, with logarithmic derivative $\partial \ell n_{G}$, we have constructed four connections (contracted with $\partial$ ) on its Lie algebra $L G$, and they all coincide:

- the purely algebraic $\partial_{L G}$, as used in the text;

- the differential-algebraic $L \partial \ell n_{G}$ (not used);

- the analytic (and actually formal) $\exp _{G}^{*}\left(\partial \ell n_{G}\right)=\partial \ell n_{G} \circ \exp p_{G}$;

- the purely algebraic $\nabla_{L G, \partial}$ (if $G$ is an almost semi-abelian $D$-group).

In what follows, as well as in the text itself, we identify them using only the notation $\partial_{L G}$, which we call the logarithmic derivative of $L G$.

The next sections of the Appendix are of a different nature. As seen in the text, our main theorem, Theorem 1.3, relies in an essential way on the ManinColeman-Chai theorem of the kernel. Although this result is well documented in the literature (see [25], [13, 12]), the topic is delicate (see [13], 5]), and it seemed useful to provide the reader with a self-contained proof of what we need. We have taken the opportunity of this exercise to rewrite the results in the language of logarithmic derivatives.

\section{J. Manin's theorem.}

In this section, we present a weak version of Manin's theorem of the kernel, in preparation for the next section on Chai's sharpening, as needed in the last step of both proofs of our main theorem; see Proposition 4.4, Section 5.1 and Section 6 of the text. The proof given here is essentially the analytic one of Coleman [13, Theorem 1.4.3, which, at this point, is close to Manin's initial proof [25].

From now on, we restrict ourselves to the proper case of an abelian scheme over $S$, as in Fact H.2 of Section H. So, an abelian variety $A / K$ is given and extended to an abelian scheme $\pi: \mathbf{A} \rightarrow S$. We have its 0 -section $\mathbf{e}$ and the pull-back $L \mathbf{A}=\mathbf{e}^{*}\left(T_{\mathbf{A} \mid S}\right)$ of the relative tangent bundle of $\mathbf{A}$ over $S$. As in the introduction to the paper, we let $\left(A_{0}, \tau\right)$ be the $K / \mathbb{C}$ trace of $A / K$. After base change to a finite cover of $S$, we may assume that it is also the $K^{\text {alg }} / \mathbb{C}$-trace of $A / K^{\text {alg }}$ and that $\tau$ is an embedding. We denote by $\mathbf{A}_{0}:=A_{0} \times S$ the abelian subscheme of $\mathbf{A}$ extending $A_{0}$.

As in Section $\mathrm{H}$, the kernel of the exact exponential sequence of analytic sheaves over $S^{a n}$,

$$
0 \rightarrow \mathcal{P} \rightarrow L \mathbf{A}^{a n} \longrightarrow \mathbf{A}^{a n} \rightarrow 0,
$$

is a local system $\mathcal{P}$ of $\mathbb{Z}$-modules of rank $2 g=2 \operatorname{dim} A$ over $S^{a n}$, which can be identified with the dual of the local system $R^{1} \pi_{*}(\mathbb{Z})$ formed by the Betti cohomology groups of the fibers $\mathbf{A}_{t}$. 
Since $\pi$ is proper, any point $y$ of $A(K)$ extends uniquely to a section $\mathbf{y} \in \mathbf{A}(S)$, and we will freely use the transition from normal to bold-face characters. On the Lie algebra level, a point $x$ of $L A(K)$ extends to a section of $L \mathbf{A}$ only over a Zariski open, but still dense, subset of $S$ (which depends on $x$ ).

Lemma J.1. Let $\mathbf{x} \in L \mathbf{A}^{a n}\left(S^{a n}\right)$. Assume that $\mathbf{y}:=\exp _{\mathbf{A}}(\mathbf{x}) \in \mathbf{A}^{\text {an }}\left(S^{a n}\right)$ actually lies in $\mathbf{A}(S)$. Then, $\mathbf{y}$ is infinitely divisible in $\mathbf{A}(S)$. In particular, there exists a positive integer $d$ such that d.y lies in $A_{0}(\mathbb{C})$.

Proof. (Manin-Shafarevich) The last statement follows from the functional MordellWeil theorem, according to which the group $A(K) / A_{0}(\mathbb{C})$ is finitely generated, or more directly, from the study of the Néron-Tate height $\hat{h}$ on $A$ attached to an ample divisor. Namely, since $y$ is infinitely divisible in $A(K)$, its height $\hat{h}(y)$ vanishes, while the divisible hull $A_{0}(\mathbb{C})^{\text {div }}$ of $A_{0}(\mathbb{C})$ in $A\left(K^{\text {alg }}\right)$ is precisely the set of points with zero height. We now prove the first statement.

Let $m$ be an arbitrary positive integer. Then $\frac{1}{m} \mathbf{x}$ is again a section of $L \mathbf{A}^{\text {an }}$ over $S^{a n}$, and we can consider the analytic section $\mathbf{y}_{m}:=\exp _{\mathbf{A}}\left(\frac{1}{m} \mathbf{x}\right)$ of $\mathbf{A}^{a n}$ over $S^{a n}$. Since $m \mathbf{y}_{m}=\exp _{\mathbf{A}}\left(m \cdot \frac{1}{m} \mathbf{x}\right)=\mathbf{y}, \mathbf{y}$ is divisible by $m$ in $\mathbf{A}^{a n}\left(S^{a n}\right)$. Moreover, $\mathbf{y}_{m}$ has moderate growth at the points at infinity of $S$, since its coordinates are algebraic over $K=\mathbb{C}(S)$. So $\mathbf{y}_{m}$ actually lies in $\mathbf{A}(S)$ and $\mathbf{y}$ is infinitely divisible in $\mathbf{A}(S)$, as was to be shown.

Now let $\tilde{A}$ be the universal vectorial extension of $A$, endowed with its unique ADG structure, and let $\partial \ell n_{\tilde{A}}, \partial_{L \tilde{A}}$ be the corresponding logarithmic derivatives on $\tilde{A}, L \tilde{A}$. As in the text, we denote by $W_{A} \simeq H^{1}\left(A, \mathcal{O}_{A}\right)^{*}$ the maximal vectorial subgroup of $\tilde{A}$. It is defined over $K$ and may be viewed as vector subspace of $L \tilde{A}$, but it is not usually a $D$-submodule of $L \tilde{A}$.

Proposition J.2 (Manin-Coleman). Let $\tilde{x} \in L \tilde{A}(K)$, and let $\tilde{y} \in \tilde{A}(K)$ with projection $y \in A(K)$. Assume that $\partial \ell n_{\tilde{A}} \tilde{y}=\partial_{L \tilde{A}} \tilde{x}$. Then there exists a positive integer $d$ such that d.y $\in A_{0}(\mathbb{C})$; in particular, $\tilde{x} \in L \tilde{A}_{0}(\mathbb{C})+W_{A}(K)$.

Proof. Shrinking $S$ if necessary, we may assume that $\tilde{x}, \tilde{y}$ extend to sections $\tilde{\mathbf{x}}, \tilde{\mathbf{y}}$ of $L \tilde{\mathbf{A}}, \tilde{\mathbf{A}}$. Then, $\partial \ell n_{\tilde{\mathbf{A}}} \tilde{\mathbf{y}}=\partial_{L \tilde{\mathbf{A}}} \tilde{\mathbf{x}}$. By the main result of Section $\mathrm{G}, \partial \ell n_{\tilde{\mathbf{A}}}\left(\exp _{\tilde{\mathbf{A}}}(\tilde{\mathbf{x}})\right)$ $=\partial_{L \tilde{\mathbf{A}}} \tilde{\mathbf{x}}$, so that

$$
\partial \ell n_{\tilde{\mathbf{A}}}\left(\tilde{\mathbf{y}}-\exp _{\tilde{\mathbf{A}}}(\tilde{\mathbf{x}})\right)=0 .
$$

In other words, $\tilde{\mathbf{y}}-\exp _{\tilde{\mathbf{A}}}(\tilde{\mathbf{x}})$ lies in the kernel $\tilde{A}^{\partial}$ of $\partial \ell n_{\tilde{A}}$, and we could continue by projecting it to a point $y_{0}$ in the differential algebraic subgroup $A^{\sharp}$ of $A$ defined in Proposition 3.9 of the text. But $y_{0}$ is a priori not defined over the differential closure $K^{\text {diff }}$ of $K$, only over the field of meromorphic functions on $S^{a n}$, and we cannot appeal to the description of $A^{\sharp}\left(K^{\text {diff }}\right)$ given at the beginning of Section 6 . So we go back to complex analysis.

By the surjectivity of the sheaf morphism $\exp _{\tilde{\mathbf{A}}}$ from the analogue $(\dagger)$ of $\left(\dagger_{A}\right)$ for $\tilde{\mathbf{A}}$, there exists a covering of $S^{a n}$ by disks $U$, and sections $\lambda_{U} \in L \tilde{\mathbf{A}}^{a n}(U)$ such that $\left.\tilde{\mathbf{y}}\right|_{U}=\exp _{\tilde{\mathbf{A}}}\left(\lambda_{U}\right)$. Two $\lambda_{U}, \lambda_{U^{\prime}}$ differ by an element in the kernel $\tilde{\mathcal{P}}\left(U \cap U^{\prime}\right)$ of $(\dagger)$, allowing us to define a cocycle $\left\{\phi_{U, U^{\prime}}:=\left(\lambda_{U}-\lambda_{U^{\prime}}\right)\right\} \in H^{1}\left(S^{a n}, \tilde{\mathcal{P}}\right)$. In view of Proposition G.1, $\partial \ell n_{\tilde{\mathbf{A}}}\left(\exp _{\tilde{\mathbf{A}}}\left(\lambda_{U}\right)\right)=\partial_{L \tilde{\mathbf{A}}} \lambda_{U}$. The relation $\partial \ell n_{\tilde{\mathbf{A}}} \tilde{\mathbf{y}}=\partial_{L \tilde{\mathbf{A}}} \tilde{\mathbf{x}}$ then implies that $\xi_{U}:=\lambda_{U}-\left.\tilde{\mathbf{x}}\right|_{U}$ lies in $\mathbb{C}$-vector space of horizontal sections of $\partial_{L \tilde{\mathbf{A}}}$ over $U$. But since $\partial_{L \tilde{\mathbf{A}}}=\nabla_{L \tilde{\mathbf{A}}}$ is the Gauss-Manin connection, this space 
$(L \tilde{\mathbf{A}})^{\partial}(U)$ coincides with $(\tilde{\mathcal{P}} \otimes \underline{\mathbb{C}})(U)$; cf. Facts H.1 and H.2. So, for each $U$,

$$
\exists \lambda_{U} \in L \tilde{\mathbf{A}}(U), \exists \xi_{U} \in(\tilde{\mathcal{P}} \otimes \mathbb{C})(U) \text { such that } \xi_{U}:=\lambda_{U}-\left.\tilde{\mathbf{x}}\right|_{U} .
$$

Since $\tilde{\mathbf{x}}$ is a global section over $S^{a n}$ (in fact, even over $S$ ), $\lambda_{U}-\lambda_{U^{\prime}}=\xi_{U}-\xi_{U^{\prime}}$, and the cocycle $\phi$ has trivial image under the natural map

$$
H^{1}\left(S^{a n}, \tilde{\mathcal{P}}\right) \rightarrow H^{1}\left(S^{a n}, \tilde{\mathcal{P}} \otimes \underline{\mathbb{C}}\right)=H^{1}\left(S^{a n}, \tilde{\mathcal{P}}\right) \otimes \mathbb{C} .
$$

This implies that $\phi$ is a torsion point in $H^{1}\left(S^{a n}, \tilde{\mathcal{P}}\right)$. In other words, on refining the covering $\{U\}$ of $S^{a n}$ if necessary, there exist a positive integer $d$ and sections $\xi_{U}^{1} \in \tilde{\mathcal{P}}(U)$ such that on all intersections $U \cap U^{\prime}$,

$$
d . \lambda_{U}-d . \lambda_{U^{\prime}}=\xi_{U}^{1}-\xi_{U^{\prime}}^{1} .
$$

In particular, the sections $\tilde{\mathbf{x}}_{U}^{1}:=d \cdot \lambda_{U}-\xi_{U}^{1}$ glue into a global section $\tilde{\mathbf{x}}^{1}$ in $L \tilde{\mathbf{A}}^{a n}\left(S^{a n}\right)$, and since the $\xi_{U}^{1}$ 's lie in the kernel of $\exp _{\tilde{\mathbf{A}}}$, we finally obtain

$$
\exp _{\tilde{\mathbf{A}}}\left(\tilde{\mathbf{x}}^{1}\right)=\exp _{\tilde{\mathbf{A}}}\left(d \cdot \lambda_{U}\right)=d . \tilde{\mathbf{y}} .
$$

Let $\mathbf{x}^{1} \in L \mathbf{A}^{a n}\left(S^{a n}\right), \mathbf{y} \in \mathbf{A}(S)$ be the images of $\tilde{\mathbf{x}}^{1}, \tilde{\mathbf{y}}$ under the natural projections. Since the exponential morphisms commute with these projections, we obtain $\exp _{\mathbf{A}}\left(\mathbf{x}^{1}\right)=d \cdot \mathbf{y}$, and these sections exactly satisfy the hypotheses of the previous Lemma J.1. Consequently, a multiple by a positive integer of $d . y$, hence also of $y$, lies in $A_{0}(\mathbb{C})$, and the main part of the proposition is proved.

Recall the notation $W_{A}$ above. To prove the last sentence and connect back with the running hypothesis $(\mathbf{H X})_{K}$ of the paper, we note:

Lemma J.3. Let $A / K$ be an abelian variety, let $F$ be a differential extension of $K$ with $F^{\partial}=\mathbb{C}$, and let $\tilde{x}, \tilde{y}$ be $F$-rational points on $L \tilde{A}, \tilde{A}$, projecting onto points $x, y$ in $L A, A$. Consider the following properties:

(i) there exists a positive integer $d$ such that $d . y \in A_{0}(\mathbb{C})$;

(ii) there exists a positive integer such that d. $\tilde{y} \in \tilde{A}_{0}(\mathbb{C})+W_{A}(F)$;

(iii) $x$ lies in $L A_{0}(\mathbb{C})$;

(iv) $\tilde{x}$ lies in $L \tilde{A}_{0}(\mathbb{C})+W_{A}(F)$.

Then, $(i) \Leftrightarrow i i)$ and $(i i i) \Leftrightarrow(i v)$. Moreover, if $\partial \ln _{\tilde{A}} \tilde{y}=\partial_{L \tilde{A}} \tilde{x}$ and if $F=K$, they all hold true.

Proof. (ii) $\Rightarrow$ (i) and (iv) $\Rightarrow$ (iii) are obvious, since $A=\tilde{A} / W_{A}$. For (i) $\Rightarrow$ (ii), notice that any point $y^{\prime}=d . y$ in $A_{0}(\mathbb{C})$ lifts to a point $\tilde{y}^{\prime} \in \tilde{A}_{0}(\mathbb{C})$, which will differ from the given $F$-rational lift $d . \tilde{y}$ by an $F$-rational point in $W_{A}$. Ditto for (iii) $\Rightarrow$ (iv). Finally, we have proved above that the two further assumptions imply (i), and it remains to show, say, that under these assumptions (ii) $\Rightarrow$ (iv). Indeed, (ii), written as $d . \tilde{y}=d . \tilde{y}_{0}+w$, together with the differential relation, the divisibility of vector spaces, and Corollary G.4 applied to $W_{A}$, implies that $\partial_{L \tilde{A}} \tilde{x}=\partial \ln _{\tilde{A}} \tilde{y}_{0}+\partial \ell_{\tilde{A}} w=\partial_{L \tilde{\tilde{A}}}(w)$, and $\tilde{x}-w$ is a horizontal vector of $\partial_{L \tilde{A}}$, rational over $F=K$, hence in $L \tilde{A}_{0}(\mathbb{C})$, in view of Corollary H.5 (ii).

\section{K. Chai's sharpening.}

Let $A / K$ be an abelian variety with universal extension $\tilde{A}$, endowed with its canonical ADG structure. We again denote by $W_{A} \simeq H^{1}\left(A, \mathcal{O}_{A}\right)^{*}$ the maximal vectorial subgroup of $\tilde{A}$ and by $U_{A}$ its maximal vectorial $D$-subgroup. They are defined over $K$ and can be viewed as vector subspaces of $L \tilde{A}$. 
As in Section 4.3 of the text, define an almost abelian $D$-group $G$ as an almost semi-abelian $D$-group with no toric part, i.e., such that $B=A$ is an abelian variety. So $G$ is a quotient of $\tilde{A}$ by a $D$-vector subgroup $V \subset U_{A} \subset W_{A}$, and $G$ is endowed with its unique ADG structure. Assuming for simplicity that $A_{0}=\{0\}$ and setting $W_{G}:=W_{A} / V$, we now present Chai's sharpening [12] of Manin's theorem, in terms of logarithmic derivatives.

Theorem K.1 (Chai). Let $G / K$ be an almost abelian D-group such that the abelian variety $A$ is traceless. Let $x \in L G(K), y \in G(K)$ satisfy $\partial \ln _{G} y=\partial_{L G} x$. Then, $x \in L W_{G}(K)$.

Proof. The proof given here is essentially the dual of the proof proposed by Chai at the end of his paper [12. Instead of using Cech cohomology as for Manin's theorem, we will describe the argument in terms of Galois cocycles. Since we need only one disk $U$, even only one point $t_{0}$ in $S^{a n}$, to define them, we will not use bold-face letters. We let the fundamental group $\pi_{1}=\pi_{1}\left(S(\mathbb{C}), t_{0}\right)$ act by analytic continuation on the local sections near $t_{0}$ of any local system over $S^{a n}$. In parallel with the kernel $\tilde{\mathcal{P}}$ of $\exp _{\tilde{\mathbf{A}}}$, we have the local systems $\mathcal{P}, \overline{\mathcal{P}}$, respectively defined as the kernels of the morphisms $\exp _{\mathbf{G}}$ and $\exp _{\mathbf{A}}$; we recall from Section $\mathrm{H}$ that they are all isomorphic. We denote by $(L \tilde{A})^{\partial} \simeq \tilde{\mathcal{P}} \otimes \mathbb{C}$, resp. $(L G)^{\partial}$, the vector spaces of analytic solutions of $\partial_{L \tilde{A}}$, resp. $\partial_{L G}$, near $t_{0}$ (an exponent ${ }^{a n}$ will be added when the context is ambiguous). As usual, we identify $V$ and the $D$-submodule $L V$ of $L \tilde{A}$. We have $V \subset W_{A}, A=\tilde{A} / W_{A}, G=\tilde{A} / V, L G=L \tilde{A} / L V$. Then

$$
\partial \ell n_{\tilde{A}} \tilde{y}=\partial_{L \tilde{A}} \tilde{x} .
$$

Since $A_{0}=0$, Manin's theorem from the previous section then implies that $\tilde{x} \in$ $W_{A}(K)$, and the conclusion $x \in W_{G}(K)\left(=L W_{G}(K)\right)$ will follow by projecting modulo $V$. Notice that two lifts differ by an element of $V(K)$, so that this assertion must be independent of the choice of $\tilde{y}$, in view of Corollary G.4. In fact, this corollary shows that its truth depends only on the projection $\bar{y}$ of $y$ to $A$, as it should be.

Let $\tilde{\lambda}$ be a local section of $L \tilde{A}^{a n}$ such that $\tilde{y}=\exp _{\tilde{A}}(\tilde{\lambda})$, and more generally, let $\mathcal{P}_{\tilde{y}}$ be the $\mathbb{Z}_{S^{a n} \text {-local system formed by all the determinations of the logarithms of }}$ all the multiples of $\tilde{y}$ in $\tilde{A}(K)$. Denote by $\Gamma_{\tilde{y}}$ the $\mathbb{Q}$-Zariski closure of the image of $\pi_{1}$, acting on $\mathcal{P}_{\tilde{y}} \otimes \mathbb{Q}$. Since $\exp _{\tilde{A}}$ is uniform on $S^{a n}$ and $\tilde{y}$ is $K$-rational, $\gamma \tilde{\lambda}-\tilde{\lambda}$ lies in $\tilde{\mathcal{P}}$ for any $\gamma \in \pi_{1}$. This expression defines a cocycle

$$
\chi \in H^{1}\left(\Gamma_{\tilde{y}}, \tilde{\mathcal{P}} \otimes \mathbb{Q}\right): \Gamma_{\tilde{y}} \ni \gamma \mapsto \chi(\gamma)=\gamma \tilde{\lambda}-\tilde{\lambda} \in \tilde{\mathcal{P}} \otimes \mathbb{Q},
$$

which represents the class of $\mathcal{P}_{\tilde{y}} \otimes \mathbb{Q}$, viewed as an extension of the local system $\mathbb{Q}_{S^{a n}}$ by $\tilde{\mathcal{P}} \otimes \mathbb{Q}$.

Further, let $N$ be the kernel of the representation $\tilde{\mathcal{P}} \otimes \mathbb{Q} \subset \mathcal{P}_{\tilde{y}} \otimes \mathbb{Q}$ of $\Gamma_{\tilde{y}}$. Since $(L \tilde{A})^{\partial} \simeq \tilde{\mathcal{P}} \otimes \mathbb{C}$ and since the connection $\partial_{L \tilde{A}}$ is fuchsian, its differential Galois group is the extension $\Gamma_{\mathbb{C}}$ to $\mathbb{C}$ of the quotient $\Gamma=\Gamma_{\tilde{y}} / N$. Also, it is a reductive group, in view of the semi-simplicity given by Corollary H.5 (ii). On the other hand, $\chi$ induces on the normal subgroup $N$ of $\Gamma_{\tilde{y}}$ a $\Gamma$-equivariant injective homomorphism

$$
\xi=\chi_{\mid N}: N \hookrightarrow \tilde{\mathcal{P}} \otimes \mathbb{Q} \subset(L \tilde{A})^{\partial} .
$$

We now study the image $\xi(N) \subset \tilde{\mathcal{P}} \otimes \mathbb{Q}$ of $\xi$. We will first show that it vanishes and then deduce from the reductivity of $\Gamma_{\mathbb{C}}$ a construction of the desired point $\tilde{x} \in L \tilde{A}(K)$. 
To check that $\xi(N)=\{0\}$, it suffices to show that it is contained in $(L V)^{\partial} \subset$ $(L \tilde{A})^{\partial}$. Indeed, $L V \simeq V$ is contained $W_{A}$, which intersects $\tilde{\mathcal{P}} \otimes \mathbb{Q}$ only at $\{0\}$, since in the projection from $L \tilde{A}$ to $L A=L \tilde{A} / W_{A}$, the local system $\tilde{\mathcal{P}}$ maps isomorphically onto $\overline{\mathcal{P}}$ (see Section I for the relation with the Hodge filtration). Now, to prove that $\xi(N) \subset(L V)^{\partial}$, consider the ADG projections $p: \tilde{A} \rightarrow G, L p: L \tilde{A} \rightarrow L G$, let $\lambda=L p(\tilde{\lambda}) \in L G^{a n}$, and set

$$
\chi_{L G}(\gamma):=L p(\chi(\gamma))=\gamma \lambda-\lambda \in \mathcal{P} .
$$

Then, $\exp _{G}(\lambda)=p\left(\exp _{\tilde{A}}(\tilde{\lambda})\right)=y$, and $\partial \ell n_{G} y=\partial \ell n_{G}\left(\exp _{G} \lambda\right)=\partial_{L G} \lambda$. But by hypothesis, this is equal to $\partial_{L G} x$, with $x \in L G(K)$, so that $\lambda^{\prime}:=\lambda-x \in(L G)^{\partial}$ is a horizontal section of $\partial_{L G}$. Since $x$ is $K$-rational, $\gamma\left(\lambda-\lambda^{\prime}\right)=\lambda-\lambda^{\prime}$, and we get

$$
\exists \lambda^{\prime} \in(L G)^{\partial}, \forall \gamma \in \Gamma_{\tilde{y}}, \chi_{L G}(\gamma)=\gamma \lambda^{\prime}-\lambda^{\prime} .
$$

(In other words, the image $\chi_{L G}$ of $\chi$ in $H^{1}\left(\Gamma_{\tilde{y}}, p(\tilde{\mathcal{P}})=\mathcal{P}\right)$ vanishes in $H^{1}\left(\Gamma_{\tilde{y}},(L G)^{\partial}\right.$ $=j(\mathcal{P} \otimes \mathbb{C})$ ), in the notation of Section I.) But $N$ acts trivially on $(L \tilde{A})^{\partial}$, hence on its quotient $(L G)^{\partial}$. Restricting $\chi_{L G}$ to $N$, we therefore obtain $L p(\xi(\gamma))=\gamma \lambda^{\prime}-\lambda^{\prime}=0$ for all $\gamma \in N$. So $\xi(N)$ does lie in $\operatorname{Ker}(L p)=L V$, and therefore $N \simeq \xi(N)=0$.

Consequently, $\Gamma_{\tilde{y}}$ coincides with $\Gamma$. In particular, the action of the differential Galois group $\Gamma_{\mathbb{C}}$ on $(L \tilde{A})^{\partial}$ lifts to an action on the affine space of solutions of the inhomogeneous equation $\partial_{L \tilde{A}}(-)=\partial \ell n_{\tilde{A}} \tilde{y}$. Since $\Gamma_{\mathbb{C}}$ is a reductive group, the corresponding PHS under $(L \tilde{A})^{\partial}$ is trivial, and the latter equation must admit a $K$-rational solution $\tilde{x} \in L \tilde{A}(K)$. We now show that $\tilde{x}$ satisfies the required conditions. On the one hand, $\partial \ell n_{\tilde{A}} \tilde{y}=\partial_{L \tilde{A}} \tilde{x}$ by construction. On the other hand, the projection $x^{\prime}$ of $\tilde{x}$ to $L G$ satisfies $\partial_{L G}\left(x^{\prime}-x\right)=\partial \ell n_{G} y-\partial \ell n_{G} y=0$. Since $\partial_{L \tilde{A}}$ is semi-simple, $x^{\prime}-x$ lifts to a $K$-rational point of $(L \tilde{A})^{\partial}$, which vanishes in view of the fixed part theorem in Corollary H.5 (ii) and our hypothesis that $A$ is traceless. So, $x^{\prime}=x$, and $\tilde{x}$ is a lift of $x$, as required.

Remark K.2. The fact that the $D$-submodule $L V$ of $L \tilde{A}$ is contained in $L W_{A}$ has played a crucial role in the proof that $N=0$. Chai presents in 12 a more general version of his theorem, dealing with arbitrary non-zero $D$-submodules of $L \tilde{A}$. His proof of this more general result actually requires an additional hypothesis, but the statement can be shown to hold in full generality; cf. [5].

We conclude with an application to sharp points. Here, $K^{\text {alg }}$ denotes the algebraic closure of the field $K=\mathbb{C}(S)$, endowed with the extension of the derivation $\partial$. Recall from the beginning of Section 6 of the text on $K^{\text {alg }}$-largeness that the sharp points of an abelian variety $A$ always satisfy $A^{\sharp}\left(K^{\text {diff }}\right)=A^{\sharp}\left(K^{\text {alg }}\right)$. We now turn to a description of the group $A^{\sharp}\left(K^{a l g}\right)$ itself. The exponent ${ }^{\text {div }}$ still denotes the divisible hull.

Corollary K.3. Let $A / K^{a l g}$ be an abelian variety and let $A_{0}$ be its $K^{\text {alg }} / \mathbb{C}$-trace. Then, the differential algebraic group $A^{\sharp}$ satisfies $A^{\sharp}\left(K^{\text {alg }}\right)=A_{0}(\mathbb{C})^{\text {div }}$.

Proof. The result is clear if $A=A_{0}$. By a standard argument, it remains to study the case when $A$ is traceless. By Proposition 3.9 of the text, the projection $\pi$ from $G:=\tilde{A} / U_{A}$ to $A$ induces an isomorphism of differential algebraic groups between the kernel $G^{\partial}$ of $\partial \ell n_{G}$ and $A^{\sharp}$. If $y \in G\left(K^{a l g}\right)$ satisfies $\partial \ell n_{G} y=\partial_{L G} x$ with $x=0$, the previous Theorem K.1 (more properly, the lifting property we actually proved 
at the level of $\tilde{A}$ ), combined with the last sentence of Lemma J.3, implies that the projection of $y$ to $A$ is a torsion point.

\section{ACKNOWLEDGEMENTS}

Both authors would like to thank the European model theory network, MODNET. The 2006 meeting in Antalya was the starting point for this research project, and subsequent MODNET meetings in Luminy (2007) and La Roche (2008) gave us the opportunity to meet and continue the collaboration in person. We also thank the referee for his comments on this paper, and in particular, for a question which led to Remarks 1.2 (ii) and 1.5 (ii) above, and to Theorem 5.3 and the discussion in Section 5.4.

\section{REFERENCES}

[1] Y. André: Mumford-Tate groups of mixed Hodge structures and the theorem of the fixed part; Compositio Math. 82, 1992, 1-24. MR 1154159 (93b:14026)

[2] J. Ax: Some topics in differential algebraic geometry I: Analytic subgroups of algebraic groups; Amer. J. Math. 94, 1972, 1195-1204. See also: On Schanuel's conjecture; Annals of Math. 93, 1971, 252-268. MR0435088 (55:8050) MR0277482 (43:3215)

[3] A. Bertapelle: Deligne's duality on the de Rham realizations of 1-motives; http://arxiv. org/abs/math.AG/0506344

[4] D. Bertrand: Schanuel's conjecture for non-isoconstant elliptic curves over function fields; Model Theory with Applications to Algebra and Analysis, vol. 1, eds. Z. Chatzidakis, D. Macpherson, A. Pillay, A. Wilkie, LMS Lecture Note Series 349, Cambridge University Press, 2008. MR2441374

[5] D. Bertrand: Manin's theorem of the kernel: A remark on a paper of C-L. Chai; June 2008.

[6] M. Brion: Anti-affine algebraic groups; J. Algebra 321, 2009, 934-952. MR2488561 (2009j:14059)

[7] D. Brownawell, K. Kubota: Algebraic independence of Weierstrass functions; Acta Arithm. 33, 1977, 111-149. MR0444582 (56:2932)

[8] J-L. Brylinski: "1-motifs" et formes automorphes; Publ. Math. Univ. Paris VII, 15, 1983, 43-106. MR723182 (85g:11047)

[9] A. Buium: Differential algebraic groups of finite dimension; Springer LN 1506, 1992. MR.1176753 (93i:12010)

[10] A. Buium: Differential Algebra and Diophantine Geometry; Herman, Paris, 1994. MR.1487891 (99k:12010)

[11] E. Cattani, P. Deligne, A. Kaplan: On the locus of Hodge classes, JAMS 8, 1995, 483-506. MR.1273413 (95e:14003)

[12] C-L. Chai: A note on Manin's theorem of the kernel; Amer. J. Math. 113, 1991, 387-389. MR1109343 (93b:14036)

[13] R. Coleman: Manin's proof of the Mordell conjecture over functions fields; L'Ens. Math. 36, 1990, 393-427. MR1096426 (92e:11069)

[14] R. Coleman: The universal vectorial biextension and $p$-adic heights; Invent. Math. 103, 1991, 631-650. See also: Duality for the de Rham cohomology of an abelian scheme; Ann. Fourier 48, 1998, 1379-1393. MR:1091621 (92k:14021) MR.1662247 (2000j:14032)

[15] P. Deligne: Théorie de Hodge II \& III; Publ. math. IHES 40, 1971, 5-57 \& 44, 1974, 5-77. MR0498551 (58:16653a); MR0498552 (58:16653b)

[16] N. Feldman, Y. Nesterenko: Number Theory IV, eds. A. Parshin, I. Shafarevich, Encyclopedia of Mathematical Sciences, vol. 44, Springer Verlag, 1998. MR.1603604 (99a:11088a)

[17] E. Hrushovski: The Mordell-Lang conjecture for function fields, JAMS 9, 1996, 667-690. MR1333294 (97h:11154)

[18] N. Katz, T. Oda: On the differentiation of De Rham cohomology classes with respect to parameters; J. Math. Kyoto Univ. 8, 1968, 199-213. MR0237510(38:5792)

[19] J. Kirby: The theory of exponential differential equations; Ph. D. thesis, Oxford, 2006.

[20] E. Kolchin: Differential Algebra and Algebraic Groups, Academic Press, New York, 1973. MR0568864 (58:27929) 
[21] E. Kolchin: Differential Algebraic Groups, Academic Press, New York, 1985. MR776230 (87i:12016)

[22] E. Kolchin: Algebraic groups and algebraic dependence, Amer. J. Math. 90, 1151-1164. MR0240106 (39:1460)

[23] P. Kowalski, A. Pillay: Quantifier elimination for algebraic D-groups, TAMS 358, 2006, 167181. MR2171228 (2006i:03051)

[24] B. Malgrange: Differential algebraic groups; Notes of an ICTP course, Alexandria, 2007. See also: Systèmes différentiels involutifs; Panoramas et Synthèses, vol. 19, SMF, 2005. MR2187078 (2006m:58004)

[25] Y. Manin: Rational points of algebraic curves over function fields; Izv. AN SSSR Mat. 27, 1963, 1395-1440, or AMS Transl. 37, 1966, 189-234. MR0157971 (28:1199)

[26] D. Marker: Manin kernels, Quaderni Math. vol 6, Napoli, 2000, 1-21. MR.1930680 (2003g:12009)

[27] D. Marker and A. Pillay: Differential galois theory III: Some inverse problems; Illinois Journal of Math. 41, 1997, 453-461. MR1458184 (99m:12011)

[28] B. Mazur and W. Messing: Universal extensions and one dimensional crystalline cohomology; Springer LN 370, 1974. MR0374150 (51:10350)

[29] A. Pillay: Some foundational questions concerning differential algebraic groups; Pacific J. of Math. 179, 1997, 179-200. MR1452531 (98g:12008)

[30] A. Pillay: Differential algebraic groups and the number of countable differentially closed fields, in Model Theory of Fields, eds. D. Marker, M. Messmer, A. Pillay, Lecture Notes in Logic 5, 2nd edition, ASL, A.K. Peters, 2006. MR2215060 (2006k:03063)

[31] A. Pillay: Algebraic D-groups and differential Galois theory; Pacific J. Math. 216, 2004, 343-360. MR2094550 (2005k:12007)

[32] A. Pillay, M. Ziegler: Jet spaces of varieties over differential and difference fields, Selecta Math. 9, 2003, 579-599. MR2031753 (2004m:12011)

[33] J. Steenbrink, S. Zucker: Variations of mixed Hodge structures I; Invent. Math. 80, 1985, 489-542. MR791673(87h:32050a)

Institut de Mathématiques de Jussieu, Université Pierre et Marie Curie, Case 247, 4, Place Jussieu, F-75252 Paris Cedex 05, France

School of Mathematics, University of Leeds, Leeds, LS2 9JT, United Kingdom 